\title{
Die vier Survey-Welten im Überblick
}

Im Folgenden werden unterschiedliche grundlegende Koordinationsrahmen in der Produktion von survey-basiertem Wissen als Survey-Welten eingeführt. Survey-Welten sind dabei keineswegs hermetisch abgeschlossene Einheiten der Wissensproduktion, welche untereinander, das heißt mit anderen Survey-Welten, keine Verbindungspunkte haben. Die folgend beschriebenen Survey-Welten sind zunächst als Idealtypen zu verstehen. Storper und Salais sprechen hier von möglichen Produktionswelten (Storper und Salais 1997, S. 26 ff.). Sie unterscheiden diese von realen Produktionswelten (Storper und Salais 1997, S. 97 ff.). Diese sind tatsächliche Koordinationsgrundlagen einer Branche, welche eine Mischung verschiedener möglicher Produktionswelten darstellen. Die hier vorliegende Untersuchung setzt das Konzept der Produktionswelt eine Stufe niedriger an als dies Storper und Salais tun. Es wird in diesem Kapitel aufgezeigt, dass die Branche der kommerziellen Schweizer Markt- und Sozialforschung auf unterschiedlichen produktionsweltlichen Grundlagen beruht.

Dadurch ergibt sich auch eine Verschiebung bei der Betrachtung von realen Produktionswelten. Diese stellen dann nicht global exportierende, aber lokal verankerte Branchen dar, sondern es sind Survey-Projekte, in welchen mögliche Produktionswelten ihre Anwendung finden. Survey-Projekte können zwar kaum sinnvoll als reale Produktionswelten bezeichnet werden, da sie zeitlich begrenzt sind. Sie sind jedoch in der vorliegenden Untersuchung der Ort, an welchem die (idealtypischen) Konventionen der möglichen Produktionswelten zur Anwendung kommen und in Kompromissen aneinander vermittelt werden müssen. Ist die konkrete empirische Untersuchungsebene bei Storper und Salais die Branche, so ist es in der vorliegenden Untersuchung das einzelne Projekt. Die Trennung zwischen möglichen und realen Produktionswelten bedeutet im Umkehrschluss auch, dass die folgend dargestellten Survey-Welten in dieser (idealtypischen) Form kaum 
einzelnen Survey-Projekten oder Survey-Firmen zugerechnet werden können. Charakteristisch sind hier Kompromisse zwischen verschiedenen Welten. Eine zentrale Leistung im empirischen Teil dieser Arbeit besteht deswegen darin, nicht nur Survey-Welten zu identifizieren, sondern das dichte Geflecht aus verschiedenen survey-weltlichen Koordinationskonventionen zuerst zu entwirren und dann erst Survey-Welten zu beschreiben. Dadurch findet ein konstanter Anpassungsprozess zwischen Empirie und Theorie statt, welcher nicht mit einer deduktiven Haltung gleichgesetzt werden kann (Dewey 2008). Diesem Kapitel kommt folglich ein hybrider Charakter zu: Einerseits findet hier ein Bezug der Produktionsweltentheorie auf den Gegenstand der Survey-Produktion statt (Storper und Salais 1997, S. 33). Es wird dadurch geklärt, wie sich Produktionswelten in der Survey-Produktion zeigen und artikulieren. Andererseits stellt dieses Kapitel auch ein empirisches Resultat der Forschung dar. Denn der Bezug des Konzepts von Produktionswelten auf den Gegenstand der Survey-Produktion gründet sich in einer umfassenden Analyse von empirischem Material. Der Bezug der Produktionsweltenperspektive auf die Survey-Produktion bedingt allerdings auch, dass an einigen Stellen Veränderungen im Hinblick auf das von Storper und Salais beschriebene Konzept der Produktionswelten vorgenommen wurden. Die in diesem Kapitel beschriebenen Survey-Welten finden wie dargestellt ein direktes konzeptuelles Äquivalent in der Theorie der Produktionswelten von Storper und Salais. Anzumerken ist, dass dieser Vergleich insofern unvollständig ist, da in der Produktionsweltentheorie der Bezug auf die hier verwendeten Konzepte wie Regimes des Engagements, Distributed Cognition und Quality-Chains fehlt. Nichtsdestotrotz weist die Theorie der Produktionswelten und der Survey-Welten einen breiten Überschneidungsbereich auf. Das Verhältnis zwischen Produktions- und Survey-Welten wird in Abb. 5.1 aufgezeigt.

Die Produktionsweltentheorie nimmt in Bezug auf die unterschiedlichen Survey-Welten nicht zwangsweise einen relativistischen Standpunkt ein. Trotzdem ist die methodische Praxis in den verschiedenen Welten sehr unterschiedlich und sie führt zu gegenseitiger Kritik, insbesondere vonseiten der "Sozialforschung“ an der „Marktforschung“. ${ }^{1}$ Wie in Kap. 2 dargestellt, sind die verschiedenen

${ }^{1}$ Die Kategorisierung in „Marktforschung“ und „Sozialforschung“ wird im untersuchten Feld selber oft verwendet. Die Kritik von der Sozialforschung an der Marktforschung wird beispielsweise durch einen akademischen Sozialforscher wie folgt geäußert: ,[...] aber ich weiß grundsätzlich nicht, das ist nicht meine Welt, ich weiß grundsätzlich nicht, ob sie überhaupt, ob sie quasi Marktforschungsstudien haben, die sich auch nur annähernd um Qualität kümmern. [...] aber es ist einfach meine Einschätzung, dass äh, dass das dort nicht so nachgefragt wird“. Die Einschätzung einer generell höheren „,Qualität“ der Survey-Forschung im Hinblick auf die „Sozialforschung“ wird dabei im Feld weitgehend geteilt. 


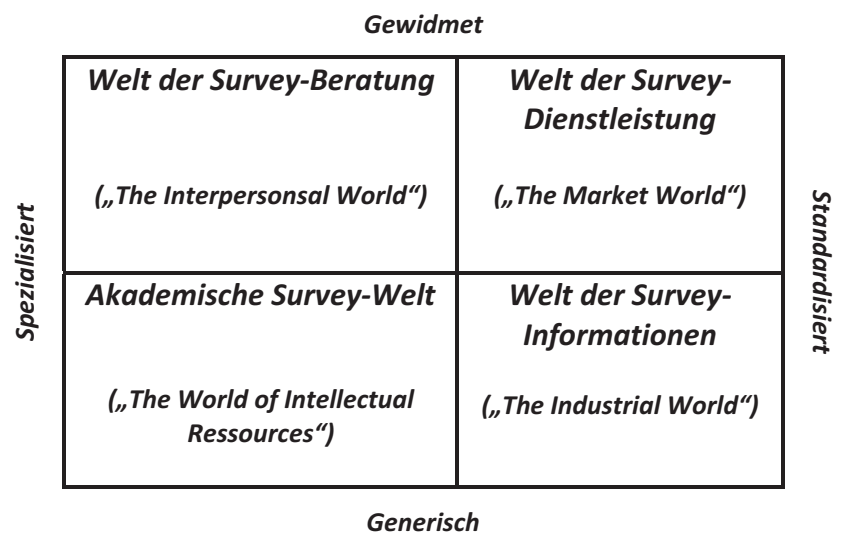

Abb. 5.1 Produktionswelten der Markt- und Sozialforschung

Elemente der Survey-Pragmatik nicht voneinander getrennt und stehen in einem regen Austausch, bzw. bedingen sich gegenseitig. Anstelle einer Kritik an methodischen Entscheidungen sollen in den folgenden Abschnitten die Ebenen des Wissensformats und der operativen Umsetzung der verschiedenen Survey-Welten idealtypisch dargestellt werden. Das Konzept der Survey-Welten ist dabei keine reine Wiederholung der Produktionsweltentheorie. In Kap. 6 wird dann die konkrete Verschränkung zwischen den verschiedenen Ebenen in konkreten Survey-Projekten nachvollzogen.

Der Verweis auf unterschiedliche Wissensformate muss in den nächsten Absätzen rechtfertigt werden. Denn der Verweis auf unterschiedliche Ontologien bedingt, dass das durch Surveys in den verschiedenen Survey-Welten produzierte Wissen nicht gegenseitig ersetzbar ist. Das Kernargument der Survey-Welt ist folglich, dass sich die Survey-Welten nicht „einfach“ im Hinblick auf die unterschiedliche methodische Praxis unterscheiden, sondern dass dieser unterschiedlichen methodischen Praxis die Orientierung an unterschiedlichen Wissensformaten zuvor läuft. Der Verweis auf unterschiedliche Ontologien in den Survey-Welten betrifft jedoch nicht nur das Wissen, sondern ist auf weitere Unterschiedsdimensionen beziehbar. Ein Beispiel hierfür stellen die Projektleiter in den verschiedenen Survey-Welten dar. Diese sind bei genauerem Hinschauen in den verschiedenen Survey-Welten nicht einfach mit unterschiedlichen Aufgaben betraut, sondern sie müssen selber unterschiedlichen Akteursformen genügen. Hat ein Projektleiter in der Dienstleistungswelt eingehende Aufträge 
zu bearbeiten, so muss er die Aufträge in der Beratungswelt selber erst definieren. ${ }^{2}$ Das „Selber-Definieren“ bedingt jedoch der Fähigkeit, ein Problem in einer Organisation eigenständig in eine Problemstellung und daran anschließend in eine Fragestellung zu übersetzen, welche abschließend in eine Empfehlung münden soll. Dies ist nicht möglich ohne eine breite Erfahrung mit organisationalen Fragen, kombiniert mit einem breiten Verständnis verschiedener Befragungsmethoden, um passgenau auf das Problem mittels geeigneter (individuell passender) Methoden eine Handlungsempfehlung erstellen zu können. Die unterschiedlichen Ansprüche an die Person des Projektleitenden bringen so unterschiedliche Akteursformen mit sich, welche nicht ohne weiteres miteinander ausgetauscht werden können. ${ }^{3}$ Die unterschiedlichen Ontologien erfordern in den Darstellungen der verschiedenen Survey-Welten eine angepasste Begrifflichkeit. So werden beispielsweise Käufer von Survey-Dienstleistungen in den verschiedenen Survey-Welten als „Auftraggeber“ in der Informationswelt und der akademischen Welt, als „Kunde“ in der Dienstleistungswelt und als „Klient“ in der Beratungswelt bezeichnet, um begrifflich möglichst passend die jeweilige Ontologie zu treffen.

Die unterschiedlichen Akteursformen wie auch die unterschiedlichen Ontologien des Wissens in den Survey-Welten trifft damit den Kern der Argumentation des Bezugs von Produktionswelten auf die Survey-Produktion: Surveys können nicht graduell in ,gute“ und „schlechte“ Surveys eingeteilt werden ohne den Bezug auf deren spezifisches Erkenntnisziel. Die Survey-Methodologie greift hier zu kurz, da sie nicht über die methodische Ebene hinaussieht und so deren pragmatische Einbettung in die Ebene des Wissensformats und der operativen Umsetzung von Survey-Projekten nicht mit in die Beurteilung der Qualität von Surveys miteinbeziehen kann.

\footnotetext{
${ }^{2}$ Mit Bezug auf Hill kann festgehalten werden, dass sich Surveys als Dienstleistungen nicht ohne die Zusammenarbeit des Auftraggebers durchführen lassen und in der Folge nicht von den Eigenheiten des Auftraggebers getrennt werden können (Hill 1999, S. 428). Die Art der Zusammenarbeit unterscheidet sich dabei jedoch grundlegend zwischen den verschiedenen Survey-Welten.

${ }^{3}$ Das Sprechen von „Projektleitenden“ ist dabei in einigen Survey-Welten notwendigerweise ungenau. Trifft dieses Konzept gut auf die Beratungswelt zu, da hier möglichst eine Person das ganze Projekt koordiniert, ist es bei Projekten der Informationswelt beispielsweise unklar, wer genau ,der“ Projektleiter ist und wer nicht. Denn hier sind neben sogenannten „Projektleitenden“" oft verschiedene weitere Personen in ein Survey-Projekt integriert. Mit „Projektleiter" wird in einem solchen Fall eigentlich ein Kollektiv beschrieben.
} 
Sämtliche Survey-Welten kombinieren Handlungsregime zu einer situativen Koordinationspragmatik. Damit ist gemeint, dass die unterschiedlichen Regime in jeder Survey-Welt als Koordinationsform auftauchen. Dies betrifft einerseits die organisatorischen Koordinationsmaßnahmen wie Koordinationssitzungen, Angebotserstellung, usw. Andererseits betrifft dies auch die methodische Umsetzung, sodass beispielsweise das Regime der Exploration prinzipiell in jeder Survey-Welt als eine Grundlage für die Planung der Befragung - insbesondere der Gestaltung des Fragebogens - miteinbezogen werden kann, um das Interesse an der Befragung zu wecken und so eine erhöhte Response-Rate in der Befragung zu generieren. Die verschiedenen Handlungsregime spielen jedoch eine unterschiedlich prominente Rolle in den verschiedenen Survey-Welten, welche im Folgenden dargestellt werden. Man könnte hier von Wahlverwandtschaften sprechen. Dies insofern, weil die verschiedenen Survey-Welten auf verschiedenen Handlungsregimen aufbauen, ohne dass die entsprechenden Konventionen mit den Handlungsregimen gleichzusetzen wäre.

Zusätzlich zur Darstellung der verschiedenen Konventionen von Survey-Welten wird in den Kapiteln zu den Survey-Welten auf die Epistemologie der jeweiligen Survey-Welt eingegangen. Epistemologie wird dabei nicht als eine rein kognitive Orientierung verstanden, welche dann als Grundlage für die Ausarbeitung einer Methodologie und einer methodischen Strategie dienen. Einer pragmatischen Epistemologie folgend (Dewey 2008) sollen vielmehr in einer Praxisperspektive Wege und Bedingungen der Wissenswerdung in survey-basierter Forschung dargestellt werden.

Das Kapitel ist folgendermaßen aufgebaut: Zuerst wird in die Eigenheiten des Forschungsgegenstandes, des Schweizer Survey-Felds, eingeführt. Hier werden organisationale Strukturen und spezifische Eigenheiten dargestellt, welche einen groben Überblick über die Schweizer Survey-Landschaft ermöglichen soll. Danach werden die vier identifizierten Survey-Welten beschrieben und dargestellt. In Abschn. 5.6 wird ein Überblick über die verschiedenen Survey-Welten geleistet. Dort werden anhand der verschiedenen identifizierten Konventionen die Survey-Welten (tabellarisch) miteinander verglichen.

\subsection{Das Schweizer Survey-Feld}

Im Folgenden wird ein Überblick über das Feld der kommerziellen Schweizer Survey-Firmen erarbeitet werden. Dies ist zentral für das Verständnis der beschriebenen Survey-Welten, da diese in eine spezifische Marktstruktur eingebettet sind. Die Darstellung dieser spezifischen „Umwelt“ erlaubt es, Heraus- 
forderungen und Probleme der verschiedenen Survey-Welten besser zu verstehen. ${ }^{4}$ Die Darstellung des Survey-Feldes dient jedoch insbesondere dazu, die in den beiden Fallanalysen vorgenommenen Analysen für den Leser verstehund einordbar zu machen, da diese oft auf die spezifische Struktur des Schweizer Survey-Marktes zurückgeführt werden können.

Eine Auffälligkeit des Schweizer Survey-Marktes ist die starke Konzentration auf den Raum Luzern. So sind einerseits die drei umsatzstärksten Schweizer Survey-Firmen in Luzern oder der direkten Umgebung angesiedelt (Siegrist 2015, S. 15). Daneben existieren diverse weitere, kleine bis mittelgroße Firmen, welche survey-basierte Forschungsleistungen kommerziell anbieten. Dies ist deswegen speziell, weil die starke Konzentration im Raum Luzern in einem Missverhältnis steht zur Bedeutung des Wirtschaftsstandorts Luzern für die Schweizer Volkswirtschaft. Die Ansiedlung von Survey-Firmen im Raum Luzern ist folglich kaum durch die erhöhte Nachfrage nach survey-basierter Forschung in dieser Region zu erklären. Aus den verschiedenen Interviews und Gesprächen mit Mitarbeitenden und Geschäftsleitenden wurde jedoch klar, dass einerseits ein Netzwerkeffekt für die Ansiedlung in Luzern verantwortlich ist, wie dies ein langjähriger Survey-Forscher auf Institutsseite ${ }^{5}$ deutlich macht:

\begin{abstract}
Also gut, ich kenne die Geschichte natürlich noch, weil ich schon lange im Geschäft bin, ja, also Luzern, deshalb entstanden, weil zuerst kam Nielsen, was ja amerikanisch, da kam der Gründer persönlich, also der Herr Nielsen, der Nielsen gegründet hat $[\ldots]$ und der kam in die Schweiz und der fand die Innerschweiz schön und hat da sein Institut gegründet. Und von Nielsen wurde dann die, das Institut für Haushaltsanalysen IHA abgespaltet, die gingen nach Hergiswil. Also es waren eigentlich Nielsenleute, die eine Konkurrenz zu den Nielsen-Zahlen aufgebaut haben, in dem man eben den Haushalt gebracht hat mit Tagebüchern statt äh, in den Detailhandelsfilialen selber nachgefragt hat und die IHA, die hat dann die GFM übernommen, das war also das erste ad-hoc Forschungsinstitut, also (erstens) Survey und qualitativ-psychologisches Institut in der Schweiz und die sind dann auch nach Hergiswil gekommen, weil die aufgekauft wurden von Zürich und dann war schon mal Nielsen und GfK hier dann gab es den, ähm Werner Wyss, der hat die Demoscope gegründet, der war einfach Luzerner, also der war in den Staaten und der war zufälligerweise Luzerner und hat gefunden, er macht ein, gründet ein Marktforschungsinstitut, und dadurch
\end{abstract}

\footnotetext{
${ }^{4}$ Ein wichtiges Element dieser Umwelt stellt auch das sog. „Survey-Klima“ dar. Dieses Konzept wird von Geert Loosveldt und Dominique Joye verwendet, um die Bereitschaft und Offenheit einer Gesellschaft für Befragungen zu bezeichnen (2016). Dieses Element der Survey-Umwelt wird jedoch im Folgenden nicht weiter thematisiert.

${ }^{5}$ D. h. bei einer Survey-Firma tätig.
} 
waren es schon mal Nielsen, ääh, IHA-GfK und Demoscope hier, dann Sepp Stofer, war Leiter der Sur, der ad-hoc Forschung, also Survey-Research bei GfK, der hat Link gegründet 81 und dadurch ist auch Link in Luzern und dann gab es noch eine ganze Reihe von Spin-Offs von der GfK, also das war der Lötscher, äh, der ist da auch irgendwo im Land draußen und Kohler und Amon [...] und Ja, die haben dann ihre, ihr Institut gegründet, und weil die alle von der GfK kamen [...]. Sind die alle in der Innerschweiz geblieben, deshalb gibts, auch [...] die AmPuls, das waren auch GfKler, Alle.

Die Einschätzung der Ansiedlung im Raum Luzern als Abfolge diverser SpinOffs von Mitarbeitenden existierender Firmen kann dabei als geteilte Perspektive des Survey-Feldes verstanden werden, da sie ähnlich durch verschiedene Interview- und Gesprächspartner dargelegt wurde. Daneben lässt sich noch eine zweite Perspektive auf die Ansiedlung in Luzern identifizieren, welche von demselben Interviewpartner geäußert wurde. Demnach sind es nicht alleine verschiedene Spin-Offs, welche direkt in der Region Luzern Firmenneugründungen vorgenommen haben. Die Treue gegenüber dem Standort Luzern wird hier durch einen langjährigen Survey-Forschers auf Institutsseite mit den spezifischen Arbeitsmarktbedingungen erklärt:

[...] nein der andere Grund, warum in Luzern, ist meine Theorie, war früher war Marktforschung ein Hausfrauenbusiness, also man brauchte viel, äh günstige Hausfrauen. Die die ganze Handarbeit gemacht haben. Vercoden, äh, Papierfragebögen umschichten, Nachfragen, sind die Fragebogen ausgefüllt, ääh, auch Telefonbefragungen am Anfang, da brauchte man ja Leute, die nicht so teuer waren, die gab es halt in der Innerschweiz eher als in Zürich. Die, die Konkurrenz von anderen Wirtschaftszweigen war nicht so groß, also man hat eher günstige Arbeitskräfte bekommen und das war sehr viel Handarbeit, nicht so hohe Wertschöpfung und deshalb ist meine Vermutung [...] haben eben nur die Luzerner Institute, kamen zum Glühen. Es gab ja schon Zürcher Institute wie die äh, IPSO, damals, noch ein paar andere kleine, Publi Test, die sind einfach nicht wirklich gewachsen. Wahrscheinlich hatten sie die Kosten und Kapazitätsprobleme. Und und deshalb ist schon die günstigeren Kostenstrukturen für, für all diese nebenamtliche Handarbeit. Das war ein Vorteil.

Die wirtschaftlich eher strukturschwache Region Luzern bietet demnach Befragungsinstituten Vorteile. Zu erwähnen ist jedoch, dass sich diese Konzentration auf den Raum Luzern in den letzten Jahren abzuschwächen scheint. Einerseits finden Verlagerungen von Firmenteilen in andere Regionen, insbesondere Zürich 
statt, andererseits wechselt beispielsweise GfK ihren Standort. ${ }^{6}$ Grundsätzlich scheint jedoch die Nähe zum Kunden eine erhöhte Bedeutung zuzukommen. Dies ist wohl nicht zuletzt in einem veränderten Trade-Off zwischen Befragungskosten und Kundennähe zu suchen, welcher durch die Abnahme der Bedeutung von telefonischen Befragungen zugunsten des Faktors Kundennähe verändert wird:

Schweiz ist klein, also Luzern ist nicht wirklich abgelegen. Also es ist nicht so ein riesen Problem. Und früher war die Wirtschaft nicht so auf Zürich fokussiert. Also am Anfang hatte ich Kunden in Zug, in Zürich, aber auch viel in Bern [Aufzählung verschiedener Kundenfirmen] also ich bin eigentlich in der ganzen Schweiz rum rumgefahren, ich war gar nicht so oft in Zürich. Und heute hat sich das ganz stark nach Zürich konzentriert. [...] [Die Region Luzern] war nicht so ein Problem, von von der Kundenbetreuung her natürlich war man gut am Mann, man hat sich sowieso mehr Zeit genommen für ein Projekt, also, ist der Firmenmarktforscher ist gern in die Schwii, ist gerne in die Innerschweiz gereist und hat man am Morgen ein bisschen über das Projekt geredet, hat man Mittaggegessen, ein gutes Glas Wein und dann war der Tag beendet [...].

Nebst der nach wie vor starken Konzentration auf den Raum Luzern ist ein zweites Charakteristikum des Schweizer Survey-Feldes zu nennen. So verfügt weder die wissenschaftliche Survey-Forschung noch die öffentliche Statistik über eine eigene feste Befragungsinfrastruktur. ${ }^{7}$ Dies hat zwei Konsequenzen. Einerseits machen Aufträge von öffentlich-rechtlichen Organisationen einen nicht unerheblichen Prozentsatz des Branchen-Umsatzes (Siegrist 2015, S. 16). Dieser Umsatz konzentriert sich jedoch hauptsächlich auf drei Firmen, wobei die GfK als größte Survey-Firma aktuell keine akademischen Surveys oder solche aus der öffentlichen Statistik durchführt. ${ }^{8}$ Gleichzeitig bleibt diese Art der Befragung auch

\footnotetext{
${ }^{6}$ Wobei unklar bleibt, was genau diesen Standortwechsel ausgelöst hat. Zusätzlich findet ein Wechsel von einem Randbezirk der Region Luzern in einen anderen, näher an Zug und Zürich gelegenen Randbezirk statt, so dass nicht wirklich von einer Abwanderung gesprochen werden kann.

${ }^{7}$ Abgesehen von kleineren Einrichtungen, welche jedoch für größere Surveys nicht geeignet sind. Zudem sind diese kleineren Einrichtungen nicht grundsätzlich für wissenschaftliche Surveys oder solche der öffentlichen Statistik zugänglich.

${ }^{8}$ Dies ist der Branchenstatistik leider nicht zu entnehmen. Dies kann jedoch den verschiedenen Beobachtungen und Interviews entnommen werden. Darüber hinaus ist es für die Übernahme von Befragungen für universitäre und amtliche Befragungen unerlässlich, über ein eigenes Telefonlabor zu verfügen mit einer notwendig hohen Kapazität für diese Art von Befragungen. Über eine solche Infrastruktur verfügt nur die überschaubare Anzahl an mittelgroßen und großen Survey-Firmen.
} 
in den erwähnten Survey-Firmen eine Nische. ${ }^{9}$ Andererseits sind akademische Survey-Projekte wie auch die öffentliche Statistik auf die Infrastruktur und eine gelingende Zusammenarbeit mit den Survey-Firmen angewiesen. Dies wird im folgenden Zitat eines langjährigen akademischen Survey-Forschers erkennbar:

Und und ich sag immer, äh, wir dürfen uns nicht auf ein Institut kaprizieren, das wäre, also das ist ein Klumpenrisiko. Das wäre das ganz ganz schlecht. Also wir müssen mehrere Partner haben und wir müssen die dann auch füttern, also, wir müssen die Aufträge schon ein bisschen verteilen, weil sonst, äh, sonst gehts dann auch nicht, oder?

Deutlich wird hier die Angewiesenheit der akademischen und auch öffentlichen Survey-Forschung auf die privatwirtschaftlich organisierten Survey-Firmen durch eine fehlende staatliche Befragungsinfrastruktur. Die Aufträge werden hier stets öffentlich ausgeschrieben und es findet eine regelmäßige Zusammenarbeit mit verschiedenen, größeren Survey-Firmen statt. Auch Survey-Firmen, welche regelmäßig für die akademische Survey-Forschung oder die öffentliche Statistik arbeiten, sind folglich auf Einkünfte aus der privatwirtschaftlichen Survey-Forschung angewiesen. ${ }^{10}$

Obwohl Auftraggeber unterschiedliche Qualitätsvorstellungen an die Survey-Firmen herantragen, existieren auch gemeinsame Anliegen des Survey-Feldes insgesamt. Eine für die Auftragnehmer und Auftraggeber gleichermaßen bestehende Herausforderung stellt die Antwortbereitschaft von befragten Personen dar. Diese wird aus der Perspektive der Survey-Praktiker einerseits durch den hohen Befragungsdruck, das heißt die Häufigkeit von telefonischen Befragungen aus Sicht der befragten Personen, bedroht. Zusätzlich werden teilweise Verkaufsabsichten durch den Einsatz von Befragungen kaschiert. Die Gemeinsamkeit dieses Problems wird durch einen akademischen SurveyForscher unterstrichen:

[...] ein gemeinsames Anliegen ist, ist die Antwortbereitschaft, und da leiden alle drunter, dass die sinkt, dass die unter anderem auch mit Direct-Marketing zu tun hat oder? All diese lästigen Anrufe, die man die man kriegt und die man auch dann

${ }^{9}$ Der Umsatz mit öffentlich-rechtlichen Organisationen macht $13,4 \%$ des gesamten Branchenumsatzes im Jahr 2015 aus (Siegrist 2015, S. 16).

${ }^{10}$ Zum gleichen Schluss kommt Erwin Scheuch für die deutsche Survey-Branche (1999). 
kriegt, wenn man diese Sternchen hat da im Telefonbuch drin hat, äh, äh, das schadet, das schadet der Branche, das schadet uns auch, oder? Also, äh, insofern gibt es dann wieder gemeinsame, auf dieser Ebene gibts dann wieder gemeinsame Interessen.

Der akademische Survey-Forscher verweist hier auf die Problematik der Antwortbereitschaft von Befragten, welche nicht alleine ein Problem für die akademische Survey-Forschung darstelle, sondern sämtliche Survey-Forscher - und damit Survey-Welten - gleichermaßen betreffe. Abgesehen von den in den folgenden Kapiteln zu beschreibenden unterschiedlichen Koordinationslogiken der verschiedenen Survey-Welten wird hier klar, dass auch gemeinsame Interessen und Anliegen der verschiedenen Survey-Welten existieren. Dies wird durch die gemeinsame Interessenvertretung der Schweizer Survey-Forscher durch den Verband Schweizer Markt- und Sozialforschung (VSMS) deutlich. Der Vorstand ist hier aus Personen aus verschiedenen Survey-Welten zusammengesetzt (VSMS 2017). Aus der Perspektive der Survey-Welten ist dieser Verband deshalb interessant, da hier gemeinsame Regeln und Best-Practices für sämtliche Mitglieder festgelegt werden müssen, welche jedoch aus unterschiedlichen Survey-Welten stammen. Die Porosität der verschiedenen Survey-Welten zeigt sich auch in Querrekrutierungen zwischen den verschiedenen Survey-Welten. So wechselten mehrere der Interviewpartner während ihrer Berufskarriere zwischen verschiedenen Survey-Welten. Survey-Welten sollten folglich nicht als abgeschlossene Systeme verstanden werden, welche sich eindeutig einzelnen Survey-Firmen, Käufern oder Akteur zuschreiben lassen. Entscheidend für die survey-weltliche Identifikation ist die tatsächlich verwendete konventionelle Grundlage der Koordination, wobei Organisationen und Akteure in der Regel auf verschiedene Konventionen als Koordinationsgrundlage zugreifen können (Salais und Storper 1992, S. 182). Dies ist aus der Perspektive der Survey-Firmen notwendig, um die verschiedenen Qualitätsansprüche von unterschiedlichen Käufern befriedigen zu können. Für die Käufer selbst ist die Kenntnis von verschiedenen Konventionen der Survey-Produktion hilfreich für ein reflexives Qualitätsmanagement des eigenen Survey-Projekts.

\subsection{Welt der Survey-Beratung}

Das Wissensformat der Beratungswelt ist die Empfehlung. Im Lichte des Konzepts der Survey-Welten bekommt der Beratungsbegriff hier eine spezifische Konnotation. Beratung wird in der soziologischen Beratungsforschung als eine Form der Kommunikation beschrieben, die auf ein Problem bezogen ist und durch eine Wissensasymmetrie zwischen Ratgeber und Ratsuchenden gekennzeichnet 
ist (Schützeichel 2004, S. 274 ff.). ${ }^{11}$ Im Hinblick auf die Survey-Welt der Beratung zeigt sich dies in einem Wissensgefälle im Hinblick auf die Fähigkeit der Fragestellungsgenerierung, wie aber auch auf die Durchführung der Survey-Befragung und die anschließende Datenauswertung und -interpretation. ${ }^{12}$ Eine Dienstleistungsmentalität wäre hier fehl am Platz, da die Survey-Firma infolge ihrer Kompetenz eine Führungsrolle übernehmen muss. Dies wird im folgenden Zitat eines langjährigen Projektleiters erkennbar:

Ich komme und sage, sie, da ist der Fragebogen. Ich orientiere, das ist er. Sie haben dazu eigentlich gar nichts zu sagen, weil ich weiß was ich ihnen geben muss, weil ich kenne die Instrumente.

In diesem Zitat wird deutlich, dass ein Ungleichgewicht zwischen Auftraggeber und Survey-Firma, bzw. dem Berater, im Hinblick auf die Kenntnisse der Survey-Produktion besteht. Die Survey-Firma kann sich deswegen nicht alleine als ausführende Instanz sehen, sondern muss aktiv den Survey-Prozess mitgestalten. Die Beratungswelt basiert folglich auf einer kritischen Einschätzung der Fähigkeiten der Survey-Auftraggeber, wie der langjährige Projektleiter weiter darstellt:

[...] aber ich komme dann wieder zurück zum Rezipienten, der die Marktforschung bezieht. Kann er überhaupt beurteilen [...], wie gut das ist?

\footnotetext{
${ }^{11}$ Im Alltagsgebrauch ist der Beratungsbegriff unweigerlich mit den transnationalen großen Beratungsfirmen wie McKinsey, Deloitte, Boston Consulting etc. verbunden. Diese wären aus der Perspektive der Survey-Welten jedoch eher der Informationswelt zuzurechnen, da hier allgemeine Modelle der Unternehmensführung verkauft werden. Vergleich für eine konventionentheoretische Perspektive auf diese Beratungsart (Schmidt-Wellenburg 2015, S. 286 ff.).

${ }^{12}$ Damit wird eine andere Interpretation der Rolle des Klienten in der Beratungswelt im Vergleich zur Konzeption des Käufers in der interpersonellen Welt bei Storper und Salais vorgenommen. Diese sehen in der interpersonellen Welt das Bedienen von individuellen Wünschen als die zentrale Koordinationsgrundlage (Storper und Salais 1997, S. 20). Dies stellt hohe Anforderungen an die Artikulationsfähigkeit des Kunden. Hier wird jedoch die Position vertreten, dass diese Fähigkeit durch die Survey-Firma alleine gemanagt werden muss. Denn bei der Produktion eines vollständig singulären Produkts (Karpik 2011) ist das eigentliche Produkt nach der Produktion ersichtlich. Diese kognitive Ambiguität lässt sich auch nicht durch eine höhere Kompetenz auf Klientenseite überwinden. Die von Storper und Salais beschriebene interpersonelle Welt stellt aus Sicht der Survey-Welten-Perspektive einen Kompromiss zwischen Beratungs- und Dienstleistungswelt dar.
} 
Surveys werden hier durch Auftraggeber nicht lediglich in Auftrag gegeben. Vielmehr wird mit dem Berater, bzw. der beratenden Organisation ein organisationales Problem besprochen und bearbeitet, welches durch die Survey-Firma in eine Fragestellung übersetzt wird, welche durch einen Survey beantwortet werden soll. Interaktion ist hier folglich Arbeit und ein essenzieller Bestandteil der Dienstleistung (Böhle 2011, S. 457). Die Konzeption der Fragestellung, des Untersuchungsdesigns und der Befragung, wie auch die Interpretation der Daten wird durch die beratende Organisation vorgenommen. Die Beratungswelt ist folglich ein „Markt für Zitronen“ (Akerlof 1970), ${ }^{13}$ da die Kundenorganisation die eingekaufte Qualität kaum bewerten kann durch das fehlende SurveyKnow-how (für eine weitergehende Analyse dieser Eigenschaft der Beratungswelt vgl. Abschn. 7.2). Koordination basiert hier maßgeblich auf Vertrauen. ${ }^{14}$ Denn für den Auftraggeber sind die einzelnen Schritte der Survey-Produktion zwar evtl. einsehbar, aber nicht zu beurteilen, da ihm dafür das notwendige Wissen zur Survey-Methodologie fehlt. Eine fehlende Transparenz im Hinblick auf die Produktionskette für den Auftraggeber ist folglich charakteristisch für diese Welt und nicht problematisch. Mit Bezug auf die Rechtfertigungsordnungen bei Boltanski und Thévenot kann hier die häusliche Rechtfertigungsordnung als die grundlegende Koordinationskonvention identifiziert werden (Boltanski und Thévenot 2007, S. 228 ff.). Boltanski und Thévenot sprechen von einem Vertrauensverhältnis in der Arbeitswelt, welche ein auf „Unaufdringlichkeit und Zurückhaltung beruhendes Klima gegenseitigen Verständnisses“" schaffe (Boltanski und Thévenot 2007, S. 231). Die Qualität der Zusammenarbeit misst sich in dieser Welt folglich maßgeblich am Vertrauen, welches durch die beratenden Projektleiter hergestellt werden kann. ${ }^{15}$ Dieses Vertrauen baut auf verschiedenen

\footnotetext{
${ }^{13}$ Akerlof thematisiert in seinem berühmten Aufsatz über den „Markt für Zitronen“ die lediglich bedingt mögliche Rationalität von Käufern von Gebrauchtwagen. Denn für den durchschnittlichen Käufer ist es nur sehr schwierig möglich gute Gebrauchtwagen von schlechten Gebrauchtwagen, welche in Amerika als „Zitronen“ bezeichnet werden, zu unterscheiden (Akerlof 1970).

${ }^{14}$ Die hier verkaufte Dienstleistung könnte folglich als singuläres Produkt bezeichnet werden (Karpik 2011, S. 20 ff.).

${ }^{15}$ Michael Florian argumentiert, dass sich das Vertrauenskonzept der EC maßgeblich auf die Welt des Hauses gemäß Boltanski und Thévenot, die projektbasierte Polis gemäß Boltanski und Chiapello und auf das Regimekonzept von Boltanski bezieht (Florian 2015). Er plädiert für eine eigenständige Theoretisierung des Vertrauensbegriffs in der EC. Dabei wird jedoch das zentrale - und aus Sicht der EC vertrauensgenerierende - Element der Prüfung für die EC unterschätzt (Boltanski und Thévenot 2007, S. 177 ff.), welche durch Bessy und Chateaureynaud durch das Konzept der „prise“ umfassend thematisiert wird
} 
Stützen auf. Zunächst ist es oft zentral, eine Empfehlung von vertrauten Personen zu bekommen, welche eine Zusammenarbeit mit einer gewissen Survey-Firma empfehlen. ${ }^{16}$ Ein wichtiges Kriterium für Vertrauen in dieser Welt ist zudem die Fähigkeit des Beraters, organisationale Probleme präzise zu identifizieren. Deutlich wir hierbei die von Boltanski und Thévenot beschriebene geringe Reichweite der infolge des Bezugs auf die häusliche Konvention zustande kommenden Qualität, welche sich auf den zwischenmenschlichen Kontakt beschränkt (Boltanski und Thévenot 2007, S. 240). Dies bedeutet, dass die hier produzierte Qualität nicht über Labels, allgemeine Normen oder Standards kommunizierbar ist, wie beispielsweise in der Dienstleistungswelt, sondern sich erst während der eigentlichen Zusammenarbeit zeigt. Die Produktqualität schlussendlich misst sich wie bereits erwähnt daran, inwiefern die auf dem durchgeführten Survey aufbauenden Empfehlungen fähig sind, die bestehenden organisationalen Probleme zu lösen.

Zentral für eine funktionierende Koordination in dieser Survey-Welt ist deswegen die persönliche Passung zwischen der auftraggebenden und der auftragnehmenden Organisation, wie in der folgenden Aussage eines langjährigen Mitarbeitenden einer Survey-Firma deutlich wird:

Man muss [...] die Nasen zusammenkriegen. Wenn man das nicht schafft, dann hat man ein Problem. Ja, die müssen einfach zusammenpassen.

Die persönliche Passung generiert in dieser Welt Vertrauen. ${ }^{17}$ Im Verhältnis zu den anderen Survey-Welten tritt hier der Projektleiter aus der Survey-Firma heraus. Projektleitende sind in dieser Survey-Welt nicht nur Angestellte. Da sie eine hohe Entscheidungskompetenz haben müssen und ein hohes Know-how in Bezug auf organisationale Probleme, stehen sie eher in der Funktion eines Partners zur Beratungsorganisation. Die Entstehung der Wirtschaftsbeziehung findet kaum anonym durch den Vergleich zwischen verschiedenen Survey-Firmen statt, da das eigene Survey-Projekt durch die auftraggebende Organisation noch gar

(Bessy und Chateauraynaud 2014). Vertrauen wird aus dieser Perspektive grundlegend durch den glaubwürdigen Bezug von Akteuren auf Konventionen generiert.

${ }^{16} \mathrm{Vgl}$. Abschn. 7.2, welches unter anderem das Marktproblem der Beratungswelt zum Gegenstand hat.

${ }^{17}$ Nicht zufällig wird die Rolle von Vertrauen, bzw. noch genereller der persönlichen Passung, in der Erstellung von Survey-Projekten primär in der Literatur zur Marktforschung thematisiert (Fankhauser und Wälty 2011; Zaltman und Moorman 1988). 
nicht umrissen werden kann (denn bei der Auftragsvergabe besteht anfänglich nur ein Problem, welches gelöst werden soll). Zweitens würde der auftraggebenden Organisation auch das Know-how für eine Kodifizierung des Auftrages auch fehlen. ${ }^{18}$

Die Notwendigkeit eines persönlichen Passungsverhältnisses zwischen Berater und Klienten verweist darauf, dass Koordination in der Beratungswelt eng mit dem Regime des Vertrauten verknüpft ist. Es ist das grundlegende Koordinationsprinzip der Beratungswelt, eine individuell auf den Auftraggeber zugeschnittene Survey-Lösung zu erstellen. Im Kapitel zur Beratungswelt wurde darauf hingewiesen, dass dies nicht ohne inhaltliche Bezugnahme von Projektleitenden auf das organisationale Problem des Auftraggebers möglich ist. In einer reflexiven Anwendung des phänomenologischen Grundsatzes des ,maximalen Willens zum Verstehen“" (Honer und Hitzler 2015, S. 17) ist es für ein gelingendes methodisches Vorgehen unumgänglich, die spezifische Situation des Klienten als Grundlage für die inhaltliche und methodische Strategie zu nehmen. Dies ist nicht möglich ohne den Miteinbezug der Spezifität und der Individualität des Gegenübers, welche regimetheoretisch einen Bezug auf das „Nahe“ des Regimes des Vertrauten darstellt. Zwei Punkte werden hieraus deutlich: Erstens ist die Beratungswelt noch weniger als andere Survey-Welten von der zwischenmenschlichen Ebene trennbar. Wie im Kapitel zur Beratungswelt dargestellt wurde, ist infolge der Wissensasymmetrie zwischen Klient und Survey-Firma Vertrauen die zentrale Koordinationsform. Dieses wird grundlegend durch den Bezug auf das Regime des Vertrauten aufgebaut. Daraus folgt zweitens, dass eine funktionale Beziehung zwischen Klient und Survey-Firma nicht ausreicht für eine gelingende Wirtschaftsbeziehung. Die beratende „Person“ wird so zu einem zentralen Qualitätsfaktor für eine gelingende wirtschaftliche Interaktion, das heißt hier spezifischer für die Produktion von Empfehlungen. Schwächer als in den anderen Produktionswelten ist die „Person“ in dieser Survey-Welt von der geforderten Akteursform zu trennen. Durch die Notwendigkeit der Nähe wird hier die beratende Person in ihrer Gänze in Anspruch genommen (Storper und Salais 1997, S. 35 ff.).

\footnotetext{
${ }^{18}$ Beispielsweise ob für ein Survey-Projekt wirklich eine Grundgesamtheit von 2000 Personen notwendig ist, oder ob die Frage nicht auch mit 1000 Personen beantwortet werden kann. Dann weiter die Frage nach den richtigen Fragen, dem Fragebogendesign, Pretests, qualitativen Ergänzungsstudien, geeigneten statistischen Auswertungsmethoden und dem Bezug der Daten auf das organisationale Problem.
} 
Der hier beschriebene zentrale Fokus auf die Person, bzw. die Persönlichkeit des Beraters, als zentraler Eigenheit dieser Survey-Welt soll jedoch nicht darüber hinweg täuschen, dass es der kollektive Bezug von Berater und Klient auf die häusliche Rechtfertigungsordnung ist, welche das Problem der Unsicherheit im Hinblick auf das Vertrauen in die geschäftliche Beziehung im Allgemeinen und in die Qualität der produzierten Produkte im Spezifischen erst löst. Die häusliche Rechtfertigungsordnung mit den zentralen Elementen der Treue, der Unaufdringlichkeit, des Vertrauensverhältnis und der Diskretion liefert erst die Grundlage für eine Koordination im Vertrauten (Boltanski und Thévenot 2007, S. 230 ff.). Es ist folglich nicht die Person, welche die Koordinationssituation als solche einrichtet, sondern die „Person“, bzw. die hier beschriebene Akteursform, kann als Folge der auf der häuslichen Rechtfertigungsordnung basierenden Situationsdefinition in der beschriebenen Art und Weise erst in der Form tätig werden.

Typische Survey-Firmen in dieser Survey-Welt sind kleine spezialisierte Firmen. Typische Produkte dieser Survey-Welt sind beispielsweise Umfragen im Rahmen eines Business Developments. Um neue Märkte zu erschließen oder die eigene Position in bestehende Märkten zu verbessern werden von der Beratungsorganisation Wissensgrundlagen mithilfe von Surveys geschaffen, welche anschließend in Handlungsempfehlungen verdichtet werden. Die räumliche Reichweite der so erhobenen Daten ist eher gering, da diese nicht standardisierten Vorgehensweisen entspringen und zudem spezifisch auf eine Kundenorganisation zugeschnitten sind. ${ }^{19}$ Die Umfrage kann sich so nicht an allgemeinen Formen orientieren, wie dies in der Informationswelt gerade das Prinzip der Informationsproduktion darstellt. Sie muss durch die Befragung neue, auf den Kunden zugeschnittene Formen erschaffen. ${ }^{20}$ Survey-Projekte sind hier folglich maßgeschneidert auf die Probleme der Kunden zugeschnitten und entspringen keinem standardisierten Angebot an Vorgehensweisen wie in der Dienstleistungswelt.

Die zeitliche Reichweite ist gegenüber der räumlichen Reichweite höher, da sich die Qualität einer Erhebung in dieser Erhebung gerade daran misst, inwiefern

\footnotetext{
${ }^{19}$ Die geringe Reichweite ist gerade charakteristisch für die häusliche Rechtfertigungsordnung (Boltanski und Thévenot 2007, S. 229).

${ }^{20}$ Vgl. zur Beratung als Forminvestition Schmidt-Wellenburg (2015). Das Beratungsverständnis von Schmidt-Wellenburg wäre in Bezug auf die Survey-Welten jedoch der Informationswelt zuzurechnen, da hier Formen in zu beratenden Organisationen auf der Basis von allg. Formen etabliert werden sollen. Die Forminvestition in der Beratungswelt orientiert sich an den Ergebnissen von Survey-Ergebnissen, welche als Basis der Forminvestition dienen.
} 
diese eine neue Perspektive auf organisationale Problemstellungen aufzubauen imstande ist. In dieser Welt erhobene Daten dienen folglich nicht „einfach“ dazu, tagesaktuelle Fragestellungen wie in der Dienstleistungswelt zu beantworten, sondern sollen vielmehr eine Umstellung der organisationalen Ausrichtung ermöglichen. Dabei können auch unkonventionelle Vorgehensweisen zum Einsatz kommen, welche kaum Eingang finden würden in ein standardisiertes Angebot der Dienstleistungswelt, ${ }^{21}$ wie ein langjähriger Senior Consultant darlegt:

Die wollten Dinge haben und es gelingt uns manchmal dann mit unseren Argumenten bessere Methodenansätze zu zeigen. Also wenn die sagen, die haben gesagt, wir wollen eine Onlinestudie, da habe ich gesagt, das findet ihr gar nicht raus. Das findet ihr online gar nicht raus, die Zielgruppenabdeckung ist [...] zu klein. Und solche Dinge holt ihr ge, wohl gescheiter mit einem Experteninterviews. Spricht mal mit dem Regierungsrat X, mit dem Journalisten da. Und mit dem [...] Bundeshauskorrespondenten dort. Und das stellen wir mal das Packet so zusammen und das ist natürlich dann für die viel Interessanter als da irgendwie ein paar [...] Fragen da ins Zeugs raus zu stellen. ${ }^{22}$

Deutlich wird in diesem Zitat eine zentrale Eigenheit der Beratungswelt. Die eigentliche Qualität zeigt sich hier erst in der direkten Beziehung zwischen Berater und Klient. Im Gegensatz beispielsweise zur Welt der Survey-Information ist die Qualität folglich nicht bereits vor dem direkten Kontakt zur Survey-Firma umfangreich abschätzbar. Wie später in diesem Kapitel beschrieben, führt diese Eigenheit der Beratungswelt zu einem eigentlichen Marktproblem dieser Survey-Welt.

Die zentrale Kompetenz, welche Projektleitende in dieser Survey-Welt haben müssen, ist die Fähigkeit, organisationale Probleme in Forschungsfragen transformieren zu können. Unumgänglich ist deswegen die Fähigkeit, das zu lösende

\footnotetext{
${ }^{21}$ Eine von mehreren Gesprächspartnern verwendete Metapher für die Arbeitsweise der Beratungswelt ist das „Schustern“ oder „Zimmern“, welches als Erschaffen von situativ angebrachten methodischen Lösungen beschrieben werden kann: „wir verstehen uns bei [Survey-Firma X] so, wir machen immer sehr kreative Dinge, wir können weg von der reinen Theorie und zimmern dann jeweils, das klingt etwas hanebüchen, aber es gibt, wir zimmern oder? Wir konfigurieren dann das Tool so, dass am meisten raus schaut".

${ }^{22}$ Erkennbar wird hier, dass bereits ein Kompromiss mit der Dienstleistungswelt eingegangen wurde. Kunden im Sinne der Dienstleistungswelt Äußern hier einen Wunsch, der dann durch den Projektleitenden abgeändert und reformuliert wird. Diese „Nachbearbeitung“ eines durch einen Kunden geäußerten Wunschs stellt dabei den beratungsweltlichen Aspekt dar.
} 
organisationale Problem gemeinsam mit dem Auftraggeber zu identifizieren. Zentral ist deswegen die Fähigkeit von Projektleitenden, aktiv auf Klienten zuzugehen. Denn einerseits kommen - wie beschrieben - die Aufträge nicht wie in der Dienstleistungswelt als anonyme Offertanfragen ins Haus, andererseits müssen sich Projektleitende in dieser Welt proaktiv um die Forschungsfrage kümmern, welche zusammen mit der Kundenfirma erarbeitet werden muss. Dazu gehört, sich im Regime des Vertrauten auf die subjektive Situation des Klienten zu beziehen. Denn charakteristisch für Klienten in dieser Survey-Welt ist gerade, dass sie nicht einen fertigen Auftrag formulieren können. Zentral ist deswegen die Problemergründung durch den Projektleitenden aus der Perspektive der methodischen Möglichkeiten. Geklärt werden muss beispielsweise, welches Ziel ein Survey erfüllen muss und welche organisationalen Stellen damit bedient werden sollen. Ein Projektleitender einer Survey-Firma verwies darauf, dass eine der Hauptherausforderungen seiner Tätigkeit darin liege, dem Kunden sein durch ein Survey-Projekt zu lösendes Problem ,,aus der Nase zu ziehen“. Es reicht hier folglich nicht, sich auf die Wünsche und Äußerungen des Klienten zu verlassen. Vielmehr kommt der Kommunikation jenseits des Verbalen ein hohes Gewicht zu (Thévenot 2011c, S. 240), da nur so Zweifeln, versteckten Problemen etc. auf den Grund gegangen werden kann. Dies bedingt einerseits ein vielfältiges Wissen über die Methode Survey, zusätzlich aber auch umfassende Kenntnisse zu organisationalen Fragen. Der Projektleiter muss also auch Erfahrungen bspw. im Entwickeln der Marktpositionierung oder der spezifischen Branche haben, das heißt inhaltliches Wissen bezüglich des Forschungsgegenstandes, um sinnvoll eine Befragung planen und umsetzen zu können, aufgrund deren er später auch Empfehlungen abgeben kann. Von Vorteil ist deswegen ein operativer Erfahrungshintergrund in Organisationen, welche mit den zu beratenden Firmen vergleichbar sind oder eine langjährige Erfahrung in der (Survey-)Zusammenarbeit mit solchen Firmen, ${ }^{23}$ wie ein erfahrener Projektleiter bei einer Survey-Firma hervorhebt:

Aber sie müssen sehen, da gibt es Marktforscher, die kommen ab der Uni, nichts gegen junge Studenten, und die betreuen dann ein 50'000 er Mandat und machen Empfehlungen, also das ist völlig Balla-Balla und das kann so nicht sein, der muss zuerst mal lernen mit dem Kunden die Fragen zu stellen und heraus zu finden, voila, wo drückt ihnen der Schuh, aber da hat er das Format nicht weil er

\footnotetext{
${ }^{23}$ Und es ist gerade dieser Erfahrungsschatz, welche die Weitergabe oder Kodifizierung des akkumulierten Know-hows schwierig macht. (Storper und Salais 1997, S. 29 f.).
} 
kein Unternehmer war, er ist ja erst Student, erst Student, drum so ein [erfahrener Marktforscher], das ist natürlich cool, der hat jahrelang im Marketing von Firmen gearbeitet. Der weiß wo der Schuh drückt, der sagt dann, ja wo wollen sie, sie brauchen doch Informationen für das, das und das, um zu entscheiden und sie brauchen nicht, äh, die jungen Studienleiter sagen, geben sie mir bitte ihre Fragen. [...], so geht das. Das geht natürlich nicht.

Es ist die Erfahrung, welche in dieser Welt garantieren soll, dass sich die Empfehlung auch auf die spezifische Situation der Organisation bezieht (Storper und Salais 1997, S. 35 ff.). Denn es gilt hier nicht, allgemeine Schlüsse aus den erhobenen Daten zu ziehen, sondern für die operativen Entscheidungsträger der Kundenorganisation verständliche und unmittelbar relevante Empfehlungen abzugeben, welche von deren Seite nicht einem weiteren Interpretationsprozess unterzogen werden müssen.

Eine Arbeitsteilung - das heißt beispielsweise die Trennung von Auftragseinholung und Auftragsbearbeitung - von Survey-Projekten wie in der Dienstleistungs-Survey-Welt ist hier erschwert, da die Weitergabe des Auftrags eben nicht über standardisierte und damit kodifizierbare Projekteigenschaften geschehen kann. Dies wird in den Aussagen eines neu eingestellten Projektleiters einer Survey-Firma deutlich:

[...] das ist wirklich der Standard bei uns, es geht alles erst raus, wenn es vier Augen gesehen haben. Jetzt diese Brandstatements natürlich nicht, weil da hätte sich jemand anders zu fest eindenken müssen, also, ich habe es [Senior Projektleiter] schnell geschickt, bevor ich es rausgeschickt habe, einfach, schau dir das, denkst du das könnte so ok sein, [...] weil er den Kunden halt schon länger kennt. Und als er dann gemeint hat, sieht super aus, hau das raus, habe ich es ihm dann auch geschickt, aber äh. Er hat danach auch gemeint, es wäre nicht nötig gewesen, dass ich es ihm schicke, weil er könne, in der Kürze. Entweder muss er sich intensiv damit beschäftigen, aber dann macht es keinen Sinn mehr, weil dann hätte er es auch selber machen können. Oder er schaut einfach mal schnell drüber und findet es dann grundsätzlich ok.

Die Spezialisiertheit und damit die Individualität des Produkts erschwert in dieser Survey-Welt eine interne Auftragsweitergabe. Zudem ist der Projektleitende notwendigerweise bekannt, da es hier nicht möglich ist, den Auftrag via standardisierte Formen (Auftragsformulare, Offertanfragen etc.) anzunehmen und zu bearbeiten. Hier zeigt sich der spezialisierte und unstandardisierte Charakter von Survey-Projekten in dieser Welt im Gegensatz zu den standardisierten Welten (Storper und Salais 1997, S. 29 f.). 
Der Investitionsmodus der Projektleitenden in dieser Produktionswelt besteht darin, sich in organisationale Fragestellungen einzuarbeiten und die eigenen Coaching-Fähigkeiten zu verbessern, das heißt insbesondere die Fähigkeit, Daten zu relevanten Aussagen transformieren zu können. Dafür ist eine Sensibilisierung für das Lesen des Vertrauten des Klienten zentral. War ein Projektleitender vorher vor allem in der Dienstleistungswelt tätig, so muss er lernen, den „,icheren“ Boden der Methodenkompetenz zu verlassen und sich auf seine Rolle als Ratgeber einzulassen. Zudem muss in die spezifische Wirtschaftlichkeit dieser Welt investiert werden. Im Gegensatz zur Dienstleistungswelt werden in dieser Welt „faire“ Preise und keine reinen Marktpreise berechnet. Obwohl die Klienten dieser Welt den Marktpreis der Dienstleistung nicht einschätzen können, müssen die Berater einen angemessenen Preis verrechnen, um nicht einen späteren Vertrauensbruch zu provozieren. ${ }^{24}$

Weiter besteht der Investitionsmodus in dieser Welt darin, das eigene Kundennetzwerk zu pflegen, um auch in Zukunft Aufträge zu bekommen. Dieser notwendige Lernprozess betrifft dabei - wie bereits deutlich wurde - insbesondere junge Projektleitende, welche sich zunächst lediglich als Methoden-Experten sehen, ${ }^{25}$ unterstreicht ein langjähriger Projektleiter einer Survey-Firma:

\begin{abstract}
Aber man muss ihn erzählen lassen, ja, also, er [...] nein, wir dürfen natürlich nicht mit, das ist auch wichtig, für einen jungen Projektleiter, dass er nicht sagt, ja wollen sie tausend oder tausendeinhundert Interviews. Wenn es eben jemand ist, der nicht viel Erfahrung hat, dass das kann er nicht beurteilen, das müssen wir beurteilen, [...] und deshalb man muss dann halt, ihn, äh, ihn, ins Gespräch kommen, um zu verstehen, was ist das effektiv das Bedürfnis, was braucht der Kunde [...] um etwas, das Richtige anzubieten, und da muss man eben die richtigen Fragen stellen und das ist nicht immer die Frage was ist ihre Zielgruppe und wie groß ist das N? Das ist ja, das muss man natürlich auch lernen.
\end{abstract}

\footnotetext{
${ }^{24}$ Boltanski und Thévenot sprechen im Hinblick auf die häusliche Welt von der Dienstbarkeit. Zentral ist es hierbei, eine bei anderen auftretende Notlage nicht auszunützen. Nur so werden die Pflichten der „Großen“ dieser Welt wahrgenommen und auch in Zukunft die Erbringung von Respekt gegenüber den „Großen“ gewährleistet (Boltanski und Thévenot 2007). Hierbei zeigt sich die starke Überschneidung zwischen der Beratungswelt und der häuslichen Rechtfertigungsordnung.

${ }^{25}$ Wobei angefügt werden muss, dass sich das folgende Zitat eher auf einen Kompromiss zwischen der Markt- und Dienstleistungswelt bezieht. In der idealtypischen Beratungswelt ist es nicht denkbar, dass jemand durch einen Studienabschluss für eine Beratungstätigkeit qualifiziert genug wäre. Es fehlt in einem solchen Fall schlichtweg an Erfahrung.
} 


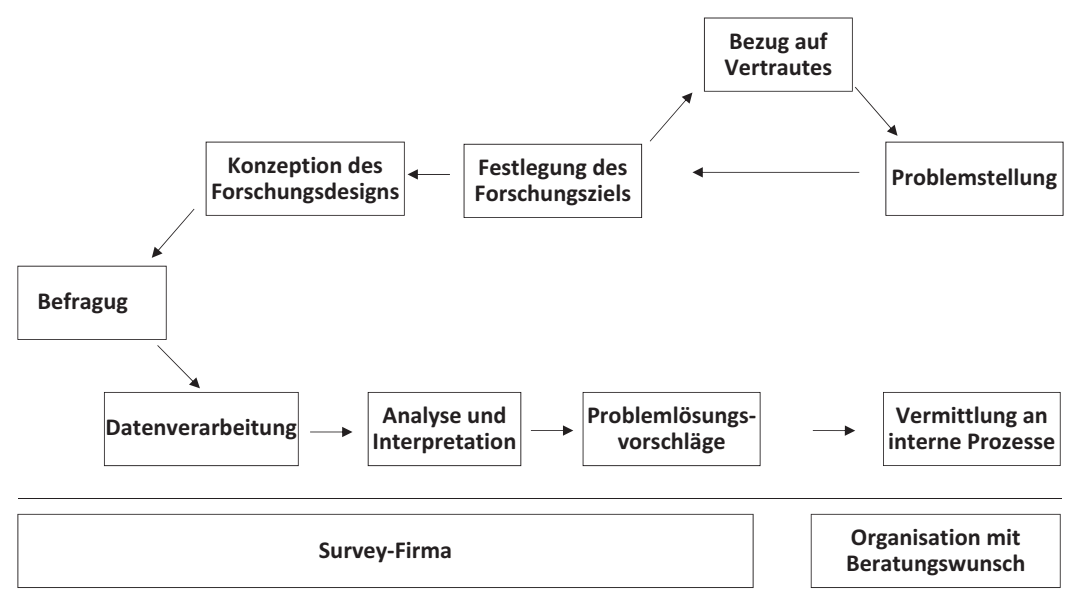

Abb. 5.2 Die statistische Kette der Beratungswelt

Der zeitliche und finanzielle Aufwand des Auftrages ist in dieser Produktionswelt kaum im Vornhinein abschätzbar. Da zu Beginn weder Fragestellung noch die verschiedenen Parameter der Befragung feststehen und zusätzlich der Aufwand der Problemlokalisierung kaum prognostizierbar ist, ist ein Gefühl für das Bedürfnis der Kundenorganisation entscheidend, um nicht am Bedürfnis vorbeizuarbeiten und in der Folge zu schwach und zu oberflächlich auf die Problemlage der Kundenorganisation einzugehen. Dies setzt wiederum den Bezug auf das Vertraute voraus und den Bezug auf eigene Erfahrungswerte.

Die Koordination zwischen Survey-Firma und Klient lässt sich idealtypisch anhand der statistischen Kette darstellen, wie dies in Abb. 5.2 vorgenommen wurde. ${ }^{26}$

\footnotetext{
${ }^{26}$ Die Darstellung ist teilweise orientiert an Biemer und Lyberg (2003, S. 27). Es zeigte sich in der empirischen Analyse, dass dieses Modell eines Survey-life-cycle als Grundlage für die Beschreibung der verschiedenen Value-Chains taugt. Jedoch gibt es je nach Survey-Welt Abweichungen von dem durch Biemer und Lyberg beschriebenen Ablauf der Survey-Forschung. Zunächst einmal in einer Abweichung von der Arbeitsteilung, andererseits aber auch im Fehlen von einzelnen Stationen des Survey-Life-Cycle wie beispielsweise des Reportings in der Beratungs- und Dienstleistungswelt.
} 
Eine zentrale Abgrenzungsposition besteht gegenüber der Dienstleistungswelt. Dies lässt sich bereits an den beiden Zitaten ablesen, in welchen die Notwendigkeit aufgezeigt wird, dass diese die „richtigen Fragen“ stellen lernen müssen. Wie in Abschn. 7.2 aufgezeigt wird, sieht sich die Beratungswelt einem grundsätzlichen Marktproblem gegenüber konfrontiert, da sie durch die Individualität der Survey-Projekte Probleme hat, ein festes Angebot zu präsentieren. Zudem ist es schwierig, die Qualität von Survey-Projekten in dieser Welt zu verdeutlichen, da einerseits den hiesigen Kunden die Kompetenz dazu schlicht fehlt und andererseits eine Qualitätsunsicherheit dadurch besteht, dass zentrale Stationen der Qualitätserstellung für den Kunden unsichtbar bleiben und nicht einschätzbar sind. Ohne gute Argumente, bzw. einen Raum, in welchen diese Argumente überhaupt wirken können, spricht in diesem Markt vordergründig alles für die Wahl der Dienstleistungswelt. Mit Bezug auf Rechtfertigungsordnungen stellt sich hier die Frage nach der Qualitätsevaluation durch Kundenorganisationen. Die Marktrechtfertigungsordnung besitzt hier eine hohe Reichweite, da sie durch die Standardisierung ein Angebot „ohne Kommentar“, das heißt ohne zusätzliche Erklärungen, erstellen und präsentieren kann, an welchem sich Kunden orientieren können (Boltanski und Thévenot 2007, S. 264 ff.). ${ }^{27}$ Die im Hinblick auf die Vermarktung auf die häusliche Rechtfertigungsordnung aufbauende Beratungswelt ist demgegenüber auf einen Kontakt angewiesen, um die Qualität zu kommunizieren. Dieses Problem wird im folgenden Zitat durch einen erfahrenen Projektleiter einer kleineren Survey-Firma hervorgehoben:

Ja, wir haben vor allem KMUs, KMUs, ich kann ein Beispiel machen bei einer [Firma A] ist ein Thema, oder eine Firma ähm, wie ähm [Firma B], das kennen sie sicher, oder wir arbeiten auch für die [Firma C], die [Firma D] und die [Firma E], aber das sind immer so Nischendinge. Und das gibt sehr gute Kundenbeziehungen, und, aber, wenn dann wieder der Ansprechpartner dort wechselt, muss man wieder bei Null beginnen.

Im Gegensatz zur Dienstleistungswelt ist die Beratungswelt folglich stärker auf persönliche Beziehungen angewiesen. Gleichzeitig ist sie jedoch der Marktkonkurrenz bei bestehenden Beziehungen weniger stark ausgesetzt, da diese Beziehungen gerade auch für die Kundenorganisation Beziehungsarbeit und damit Aufwand voraussetzt. Das zentrale Problem der Beratungswelt stellt folglich der

\footnotetext{
${ }^{27}$ Storper und Salais sprechen davon, dass der Kunde seine Wünsche in der universellen Sprache der Standardisiertheit ausdrücke (Storper und Salais 1997, S. 20).
} 
„foot in the door“ dar. Die Konkurrenz zwischen Beratungs- und Dienstleistungswelt liegt maßgeblich im höheren Preis der Beratungswelt begründet. Dieser entsteht dadurch, dass der Aufwand für das interaktive Herstellen der Forschungsfrage mitberechnet werden muss. Zusätzlich besteht jedoch ein höherer Aufwand darin, dass die Effizienz der Survey-Produktion in dieser Welt grundsätzlich kleiner ist. Dies liegt einerseits in den notwendigerweise höheren Fähigkeiten der Projektleitenden begründet, welche sich nicht nur im Hinblick auf sozialwissenschaftliche Methoden auskennen, sondern ebenfalls auch inhaltliches Wissen haben müssen. Andererseits verunmöglicht die Spezifität der Aufträge eine an einer Economies of Scale orientierten Abwicklung der Aufträge. ${ }^{28}$ Um dennoch auf dem Markt auch preislich einigermaßen konkurrenzfähig zu sein, wird insbesondere von mittelgroßen, aber auch kleineren Survey-Firmen ein Kompromiss zwischen der Beratungs- und der Dienstleistungswelt geschlossen, wie ein Junior Consultant einer Survey-Firma deutlich macht:

Und dann gibt es eigentlich uns, so ziemlich in der Mitte alleine und dann kommen viele kleine. Und eben das Argument ist halt wirklich das, wir können sozusagen, wir können das was die Großen können, einfach an allen Arten von Studien, die wir auch können. Wir können aber zusätzlich was die Großen eben weniger können, diesen persönlichen Service bieten, den die Großen wahrscheinlich weniger können. Du musst dann aber auch halt bereit sein, dafür einen leicht höheren Preis in Kauf zu nehmen. Die großen zwei können natürlich ja bei Telefonstudien immer ein bisschen einen anderen Preis offerieren, weil sie einfach allein durch die ääh, das Business of Scale, also allein durch die die Masse, die sie verarbeiten, hat es tiefere Preise, ähm veranschlagen können, pro Interview.

\footnotetext{
${ }^{28}$ Dies insbesondere durch eine fortgeschrittene Arbeitsteilung. Diese kann jedoch ein Eigenleben führen und in der Folge Probleme bereiten, wie folgende Aussage durch einen langjährigen Senior Consultant einer kleineren Survey-Firma zeigt: „Die [Anm. RV: die quantitativ ausgerichteten Marktforscher] wollen das gar nicht abgeben, die machen das alles selber. Und äh, ich kenne ja das, ich war ja selber Quantiforscher, ich habe ja meinen Leuten auch gesagt, das ziehen wir Quanti durch, weil wir müssen ja das Budget erreichen. Dann machen sie keine Quali mehr, obwohl es Sinn gemacht hätte und dann kommt das andere dazu, wenn sie sich noch gut verstehen mit dem Quali-Menschen, dann macht der eine das und der andere das und die treffen sich mal in neutralem Gebiet und koordinieren die ganze Geschichte mit sich selber, aber wir machen das bei uns selber, bei uns kommt dann ein globaler Forscher und macht beides. Der kann beides. Das ist natürlich ein Unterschied und da kriegen sie natürlich viel mehr raus. Das läuft dann alles über einen SingleSource-Ansatz, über eine einzige Person [...].“.
} 
Deutlich wird in diesem Interviewausschnitt der graduell unterschiedliche Kompromiss im Hinblick auf die Beratungs- und die Dienstleistungswelt und zusätzlich bereits der Informationswelt, ${ }^{29}$ welcher mit der Größe der Survey-Firma einhergeht. Deutlich wird zudem auch der Trade-Off zwischen dem Preis und der spezifischen Ausrichtung an individuellen Problemen von Organisationen.

Gleichzeitig formuliert die Beratungswelt jedoch auch Kritik an anderen Survey-Welten, insbesondere an der Dienstleistungs- und in schwächerem Masse an der Informationswelt, was wiederum durch die Nähe zwischen Beratungs- und Dienstleistungswelt im Hinblick auf deren geteilten, gewidmete Ausrichtung im Sinne Storper und Salais begründet ist (Storper und Salais 1997, S. 33). Kritisiert wird, dass die Dienstleistungswelt die Fähigkeiten der Kunden oft überschätze, bzw. durch fehlende „echte“ Beratungskompetenz in eine Position zwinge, die die Survey-Kompetenzen der Kunden übersteige. Eine an der Dienstleistungs- und Informationswelt gleichermaßen geäußerte Kritik besteht darin, dass ein fehlendes Eingehen auf die spezifische Situation des Kunden moniert wird.

In einer zusammenfassenden Betrachtung auf Basis einer pragmatischen Epistemologie (vgl. hierfür Abschn. 2.5.5) dieser Survey-Welt lässt sich festhalten, dass die Produktion von Empfehlungen auf einer Ko-Konstruktion zwischen organisationalen Problemen und Beratern basiert. Ein zentraler Faktor dabei ist das Vertrauen zum Berater, denn erst dieses Vertrauen ermöglicht die notwendige Vorgehensfreiheit bei der Identifikation des organisationalen Problems und der anschließenden Erarbeitung einer methodischen Lösung für die Behebung dieses Problems. Dieses Vertrauen ist notwendig, da dem Klienten hier die notwendige Fachkenntnis fehlt, um die Qualität des Vorgehens des Beraters selber beurteilen zu können. Zentral ist ebenso die Erfahrung des Beraters. Hier sind nicht die methodischen Kenntnisse angesprochen - welche in sämtlichen Welten die Grundlage für weiteres Handeln darstellen - sondern die Kompetenz in Bezug auf die Identifikation des organisationalen Problems und dessen Übersetzung in ein Forschungsdesign. Hierfür ist ein Engagement im Vertrauten notwendig, um eine Innensicht erzielen zu können. In Abb. 5.3 wird die Epistemologie der Beratungswelt zusammenfassend dargestellt.

Deutlich wird hier, dass es die Ko-Konstruktion zwischen dem Vertrauen, dem Erfahrungsschatz des Beraters und dem Engagement im Vertrauten einerseits und

\footnotetext{
${ }^{29}$ Welche sich wie in Abschn. 5.4 dargestellt wird, gerade dadurch auszeichnet, dass statistische Daten bereits zum Kaufzeitpunkt bestehen und so eine Individualität der Erhebung im Vornhinein ausgeschlossen ist und sich die Erhebung per Prinzip an einer breiten, anonymen Kundschaft ausrichten muss.
} 


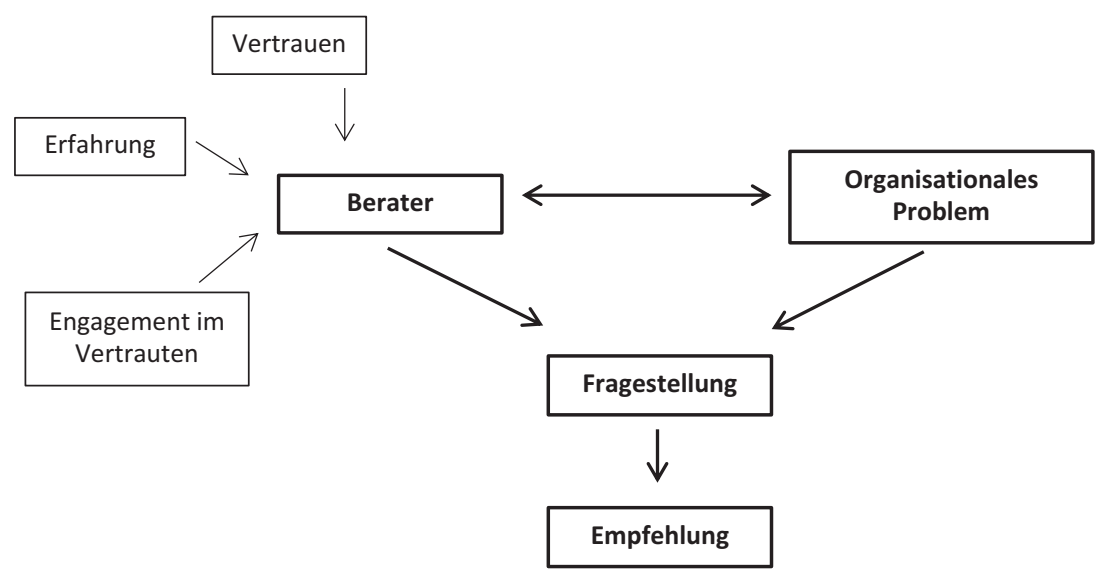

Abb. 5.3 Epistemologie der Beratungswelt

dem organisationalen Problem andererseits ist, welches zu einer Fragestellung in dieser Welt führt. Es ist schlussendlich diese idealtypisch dargestellte Epistemologie, welche die Produktion des spezifischen Wissensformats dieser Welt, der „Empfehlung“, ermöglicht.

\subsection{Welt der Survey-Dienstleistung}

Das Wissensformat der Dienstleistungswelt ist die Entscheidungsgrundlage. Kundenorganisationen kaufen eine Survey-Dienstleistung ein, um eine Grundlage für eigene operative Entscheidungen zu erhalten. Die Qualität der Survey-Dienstleistung bemisst sich folglich daran, wie stark sie in der Lage ist, als Entscheidungsgrundlage zu fungieren. Entscheidend ist folglich, dass ein Angebot erstellt wird, welches die individuellen Wünsche der Marktteilnehmer einerseits möglichst umfassend repräsentiert, andererseits eine effiziente Auslastung der eigenen Infrastruktur ermöglicht, um das Angebot auch preistechnisch attraktiv anbieten zu können. Anders als in der Beratungswelt tritt die Survey-Firma in dieser Welt nicht als Experte auf, sondern als Dienstleistungs-Unternehmen mit einem standardisierten Angebot. Die Qualität bemisst sich also nicht an der Beratung des Kunden, sondern an der Schnelligkeit und dem Preis, mit welcher Survey-Dienstleistungen erbracht und verkauft werden (Storper und Salais 1997, S. 34 f.). Der Preis der Dienstleistung richtet sich nach vorab festgelegten 
Kriterien. Der Kunde kauft also Produkte nach einem festen Preis ein, wobei Abweichungen von der vorgängig definierten Survey-Dienstleistung sich in Mehrkosten niederschlagen. ${ }^{30}$ Hingegen trägt die Survey-Firma hier die Verantwortung, zum vereinbarten Preis auch selber profitabel wirtschaften zu können. Dies wird in der folgenden Aussage eines langjährigen Projektleitenden während einer Koordinationssitzung zwischen einem Auftraggeber und einer Survey-Firma deutlich:

(P1): Du willst ja eine Leistung, du willst ja eine Leistung, oder? Du willst ja wissen, was ist die Leistung und wir sagen, was ist die Leistung, wo wir erbringen und darum haben wir die so eigentlich erbringen in dem Sinne eigentlich, wir können ja nicht danach kommen, jaa, es tut uns leid, jetzt ist es ein bisschen mehr, es ist jetzt nicht so gut gelaufen [...] Wir müssen leider noch mehr Geld haben [...]

(P2): Darum müssen wir das Risiko ein bisschen mitkalkulieren.

Die Konkurrenz um Preis und Schnelligkeit erfordert jedoch Qualitätssicherheit (Eymard-Duvernay 1989). Dieses Argument lässt sich auch umkehren. Ist der Preis zentral für eine Dienstleistung, dann ist die Einführung von standardisierten, im entsprechenden Markt breit verständlichen Qualitätsmaßen notwendig. Im Vergleich zur Beratungswelt funktioniert die Marktkoordination folglich nicht über Vertrauen, sondern über Qualitätsmaße, im Gegensatz zur akademischen Welt und zur Informationswelt jedoch nur über wenige. Für das Funktionieren des Marktes ist es notwendig, dass die verwendeten Qualitätsmaße eine hohe Reichweite aufweisen. Folglich muss sich die Ausgestaltung dieser Maße an der Survey-Kompetenz des durchschnittlichen Käufers ausrichten.

Die Dienstleistungswelt basiert auf einem öffentlichen Angebot von verschiedenen Survey-Dienstleistungen. An dieses Angebot werden zwei elementare Anforderungen gestellt. Einerseits muss dies für eine breite Masse an Kunden verständlich sein, d. h. das Anforderungsniveau im Hinblick auf die Surveykompetenz der Kunden darf nicht zu hoch sein, da sonst Kundenschichten wegbrechen, welche nicht über die notwendige Surveykompetenz verfügen. Andererseits kann das Angebot an Dienstleistungen nicht zu umfangreich sein, da dies die in dieser Welt angestrebten Economies of Scale gefährden würde. Zentral ist für diese Welt des-

\footnotetext{
${ }^{30}$ Hier zeigt sich ein Unterschied zur Informationswelt, in welcher der Endkunde, bzw. der Endnutzer, ein fertiges Produkt kauft. D. h. in der Informationswelt sind die Daten bereits erhoben, wenn der Endkunde sie kaufen will. Dadurch gibt es dort kein finanzielles Risiko für ihn.
} 
wegen ein breit verständliches Marktangebot, welches sich auf wenige Qualitätskriterien beschränkt, welche eine möglichst hohe Reichweite im Markt besitzen (Storper und Salais 1997, S. 35). Ein zentrales Qualitätsmaß ist in dieser Welt die Stichprobengröße. Diese wird meistens quotiert anhand der Kriterien Geschlecht, Alter und teilweise Region erhoben. ${ }^{31}$ Als repräsentativ gilt eine Erhebung in dieser Welt dann, wenn gemäß dieser Quotierungen erhoben wurde. ${ }^{32}$

Dabei ergeben sich verschiedene Probleme, da diese Welt nicht auf das Stichprobenregister des Bundesamtes für Statistik (BfS) zugreifen kann..$^{33}$ Ein weiteres zentrales Qualitätsmaß ist zudem der Mode, durch welchen die Befragung durchgeführt wurde. Die Erreichbarkeit der Zielgruppe ist ein weiteres oft verwendetes Qualitätsmaß in dieser Welt.

Die Dienstleistungswelt unterscheidet sich dadurch von der Beratungswelt in Bezug auf die Kodifizierbarkeit und die Unsicherheit der wirtschaftlichen Austauschbeziehung. Im Hinblick auf beide Dimensionen ist die Dienstleistungswelt zwischen der Beratungs- und der Informationswelt angesiedelt. Trotz eines vergleichbaren Angebots in der Dienstleistungswelt besteht für den Kunden in der Dienstleistungswelt eine Unsicherheit hinsichtlich des tatsächlich gelieferten Produkts, da kein vereinheitlichtes Angebot wie in der Informationswelt besteht. Die Angebote der verschiedenen Survey-Firmen können sich folglich untereinander unterscheiden, was die Vergleichbarkeit und dadurch die Qualitätseinschätzung für den Kunden erschwert.

Dies macht auf Seiten der Kundenorganisation ein Wissen notwendig, selbstständig Survey-Projekte abschätzen und planen zu können. Weiter folgt daraus, dass sich das Angebot - wie die Qualitätsmaße - am Surveywissen des „,durchschnittlichen“ Kunden ausrichten muss, um überhaupt kompetent eingekauft werden zu können. Qualität bemisst sich folglich auch an der Passung zwischen dem (standardisierten) Angebot und den Wünschen der (entindividualisierten)

\footnotetext{
${ }^{31}$ Wobei diese Quotierung in der Erhebung auch geöffnet werden kann, d. h. die Quotierung wird aufgegeben. Der Grund dafür liegt in der Verringerung der Suchkosten.

${ }^{32}$ Es werden in dieser Welt nicht exklusiv Quotenstichproben verwendet. Aus Budgetgründen wird in dieser Welt jedoch sehr oft darauf zurückgegriffen.

${ }^{33}$ Konventionentheoretisch lässt sich dies durch die fehlende Bezugsmöglichkeit dieser Welt auf die staatsbürgerliche Konvention begründen. Der Zugang zum Stichprobenregister des BfS ist einerseits für amtliche Stellen gewährleistet, andererseits für wissenschaftliche „Forschungsvorhaben von nationaler Bedeutung“ (Schweizerischer Bundesrat 2008). Dadurch wird die staatsbürgerliche Rechtfertigungsordnung als Bezugspunkt der Rechtfertigung für die Datenerhebung durch das Stichprobenregisters deutlich, was markweltlich orientierte Welten wie die Dienstleistungswelt ausschließt.
} 
Kundschaft, wie gut also Kunden ihre Survey-Bedürfnisse im Angebot einer Survey-Firma wiederfinden. Storper und Salais folgend ist der Kunde in dieser Welt der durch die neoklassische Ökonomie propagierte rationale Akteur (Storper und Salais 1997, S. 21). Im Hinblick auf Survey-Welten zeigt sich hier jedoch, wie voraussetzungsvoll diese Annahme ist. Denn um rational handeln zu können, ist aufseiten der Kunden ein grundlegendes Survey-Wissen notwendig. ${ }^{34}$

Aus der Perspektive der Regimetheorie von Thévenot basiert ein Survey-Projekt in dieser Welt maßgeblich auf dem Regime des Plans. Dies zeigt sich einerseits daran, dass operative Probleme methodisch ausgeleuchtet und keine öffentlichkeitswirksamen Daten produziert werden sollen. Im Gegensatz zur Beratungswelt wird aber deutlich, dass sich die Koordination im Plan in die Koordination zwischen Kunde und Survey-Firma weiterzieht. Durch das beidseitig vorhandene (Basis-)Wissen über die technischen Grundlagen der Survey-Forschung ist es möglich, über die Umsetzungsmöglichkeiten des Survey-Projekts zu sprechen. Für Projektleitende der Survey-Firma ist es folglich nicht notwendig, auf das Vertraute des Klienten zuzugreifen und das ,eigentliche“ Problem zu identifizieren. Die Festlegung des Survey-Projekts erfolgt direkt durch die Klärung des Wie, Wo, Wann etc. des Survey-Projekts. Eine Kompetenz der in dieser Welt tätigen Projektleitenden stellt deswegen die Ausrichtung des Angebotes an organisationalen Realitäten dar. Dies kann sich beispielsweise darin zeigen, dass sich Kategorien von Erhebungen direkt an etablierten Management-Konzepten ausrichten. Grundsätzlich orientiert sich die hier produzierte Qualität von Surveys daran, wie gut sie als Verlängerung von organisationalen Fragestellungen und damit organisationalen Realitäten funktionieren.

Die Fragestellung, die Konzeption der Methodik und des Befragungsinstrumentes wird von der Kundenorganisation erstellt. Dieses Forschungsdesign wird jedoch unter Miteinbezug des Marktangebots finalisiert. Die tatsächliche Forschung wird folglich durch das Marktangebot mitstrukturiert. Denn in einer idealtypischen Ausprägung dieser Welt existieren standardisierte Angebote, welche nicht geändert werden. Im Hinblick auf die Arbeitsteilung übernimmt die SurveyFirma lediglich die Befragung und die folgende Lieferung der Rohdaten. Die Interpretation der Daten obliegt wiederum der Kundenorganisation. Ein erfahrener

\footnotetext{
${ }^{34}$ Wie in der Beratungswelt ausgeführt wurde, ist dies eine Fähigkeit, welche nicht grundsätzlich vorausgesetzt werden kann. Entsprechend kann es zu methodischen Problemen führen, wenn Kunden trotzt geringer methodischer Survey-Kenntnisse Produkte der Dienstleistungswelt kaufen.
} 
Projektleitender aus der Beratungswelt fasst die Funktionsweise der Dienstleistungswelt wie folgt zusammen:

Drum, das sind Feldinstitute [Anm. RV: Gemeint sind große Survey-Firmen], sie müssen sehen, so eine [Firma X] macht zur Hälfte, machen die Auftrags, das ist Feldarbeit wie wir sagen, Produktionsarbeit, das ist gar keine Beratung. Die liefern den Kunden einfach ihre, Fragebogenantwort, Antworten auf ihre Fragebogen.

Deutlich wird hier, dass in der idealtypischen Dienstleistungswelt die SurveyFirmen lediglich durch das Zurverfügungstellen der Infrastruktur und deren Artikulation in einem auf die Bedürfnisse der anonymen Kundschaft zugeschnittenen Angebot Profit erwirtschaftet wird. Die methodische Kompetenz und Steuerung muss folglich durch die Kunden ausgeübt werden, während Projektleiter hier lediglich im Hinblick auf die Nutzung der Infrastruktur beratend tätig sind.

Im Hinblick auf die Projektleitenden selbst lassen sich survey-weltlich spezifische Konventionen der Arbeit identifizieren (Storper und Salais 1997, S. 57 ff.). ${ }^{35}$ Die Kompetenz von Projektleitenden in dieser Survey-Welt besteht darin, eine effiziente Koordination zwischen den Wünschen der Kundenorganisation und den internen Abläufen der Survey-Firma gewährleisten zu können. Projektleitende sollen also die Wünsche der Kundenorganisation so gut es geht befriedigen. Sie müssen sich jedoch gleichzeitig in Acht nehmen davor, Wünsche befriedigen $\mathrm{zu}$ wollen, welche nicht in die standardisierten Abläufe der Survey-Firma passen. Denn diese können massiv Mehraufwand verursachen und dadurch die Profitabilität des Survey-Projekts schnell gefährden. Der Investitionsmodus für Projektleitende liegt in dieser Survey-Welt darin, interne Abläufe besser zu verstehen, diese effizienter zu gestalten und das Angebot an Survey-Dienstleistungen so zu gestalten, dass dies auch in Zukunft den Wünschen der Kunden entspricht. Hierfür müssen die Bedürfnisse der Kunden ermittelt und in ein Marktangebot übersetzt werden. Anders als die Beratungswelt orientiert sich die Dienstleistungswelt folglich nicht an den Bedürfnissen des individuellen Kunden, sondern an den Wünschen eines anonymen Marktes (Storper und Salais 1997, S. 35). Typisch ist in dieser Welt eine Aufgabenteilung zwischen mehreren Personen, welche jedoch von der Größe der Unternehmen abhängig ist. So kommt es beispielsweise in größeren Survey-Firmen vor, dass

\footnotetext{
${ }^{35}$ Storper sprechen dann jedoch auch von unterschiedlichen Kompetenzen, über welche Arbeiter in verschiedenen Produktionswelten verfügen müssen (Storper und Salais 1997, S. 59 ff.).
} 
unterschiedliche Personen Aufträge einwerben und diese dann internen Projektleitenden zuteilen, worauf ein Junior Consultant einer Survey-Firma hinweist:

Im Endeffekt weißt du zum Teil auch gar nicht, wer das jetzt tatsächlich das effektiv gemacht hat, also ich, mein [Senior Projektleiter] ist gegen außen die Kontaktperson für sehr viele Kunden. Ahmm. Die effektive Projektleitung macht aber meist jemand aus seinem Team. Und nicht er selber. Das heißt als Kunde siehst du zum Teil gar nicht, wer dass das jetzt tatsächlich gemacht hat.

Die Arbeitsteilung bedingt interne Formen der Auftragsbearbeitung und -weitergabe. Dadurch werden Arbeiter - infolge formalisierbarer Kompetenzen - in einem gewissen Maße austauschbar. Dies im Gegensatz zur Beratungswelt, in welcher mit dem „Austausch“ eines Projektleiters unweigerlich ein Teil des Unternehmens ausgetauscht würde.

Daten kommt in dieser Welt eine mittlere räumliche Reichweite zu. Einerseits sind sie auf die Organisation zugeschnitten, andererseits ist durch das standardisierte Angebot der Survey-Firma eine gewisse Vergleichbarkeit gewährleistet, so dass die Survey-Firma die Daten bereits in einen Kontext stellen und Konkurrenzvergleiche vornehmen kann. Die zeitliche Reichweite der Daten ist allerdings gering. Denn das Ziel der Erhebungen in dieser Welt stellt die Produktion von Antworten auf tagesaktuelle Fragestellungen dar. Mit der Beantwortung der Fragestellung verlieren Daten in dieser Welt schnell wieder ihren Wissenswert, da sie nicht auf die Erhebung grundlegender Mechanismen sondern tagesaktuellen Wissens abzielen. Ein typisches Produkt dieser Welt stellen Omnibus-Befragungen her. Hier besteht für den Einkäufer die Möglichkeit, von ihm vorgegebene (wenige) Fragen die Schweizer Bevölkerung befragen zu lassen. ${ }^{36}$ Verschiedene Organisationen können sich in eine solche Befragung einkaufen. Abb. 5.4 zeigt die idealtypische statistische Kette der Dienstleistungswelt auf.

Sichtbar wird in dieser Quality-Chain die Ausrichtung an einem Marktangebot. Damit geht eine Standardisierung des von den Survey-Firmen erstellten Angebots einher. Diese Standardisierung bezieht sich dabei nicht wie in der Informationswelt auf die erhobenen Kategorien - welche in dieser Welt ohne Berücksichtigung des individuellen Kunden erfolgen, da die Erhebung vor dem eigentlichen Kundenkontakt stattfindet. Die Standardisierung bezieht sich vielmehr auf die angebotenen Methodiken und Forschungsstrategien, welche im

\footnotetext{
${ }^{36}$ Die Stichprobe wird dabei in den meisten Fällen auf der Basis des Telefonbuchs und Random Digital Dialing gezogen.
} 


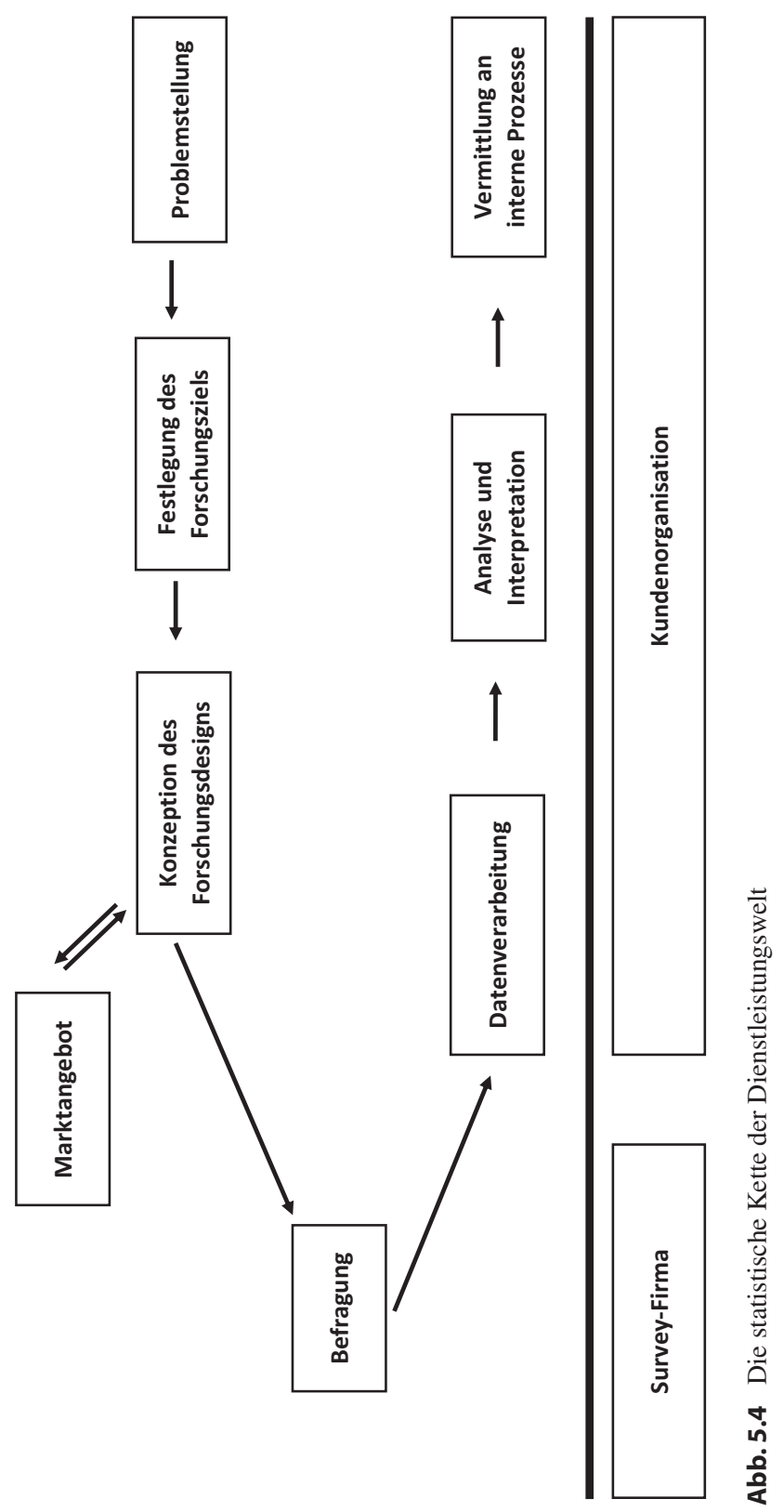




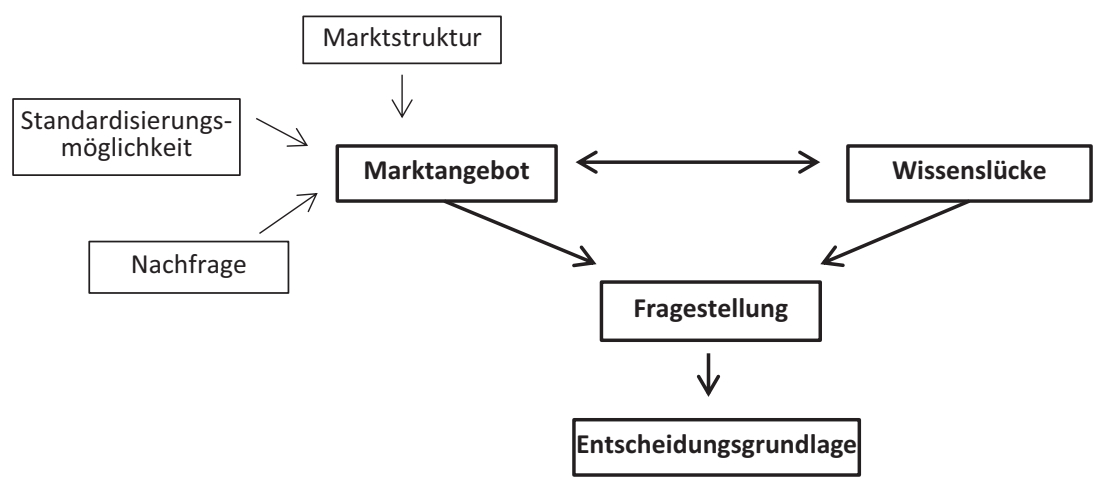

Abb. 5.5 Epistemologie der Dienstleistungswelt

Vergleich zur Beratungswelt für eine breite Kundschaft interessant sein müssen und nicht für jeden Kunden individuell festgelegt werden.

Kritik aus der Dienstleistungswelt richtet sich vor allem an die Beratungsund die Informationswelt. Die Beratungswelt wird für ihre problematische Vermarkt- und Sichtbarkeit kritisiert. Aus Sicht der Dienstleistungswelt ist dies ein Problem, da so für die Kunden kein sichtbares Angebot existiert. Zudem ist eine effiziente Abwicklung der Abläufe so nicht möglich, da die Wünsche der Kunden nicht durch ein vordefiniertes Angebot kanalisiert werden. Dies verunmöglicht aus der Perspektive der Dienstleistungswelt einen kompetitiven Marktpreis für eigene Angebote. Die Informationswelt wird für ihr fehlendes Eingehen auf die Kundenwünsche kritisiert. Daraus können Daten resultieren, welche die spezifische Situation des Kunden zu wenig reflektieren. Zudem besteht in dieser Welt das Risiko einer zu starken Preisfokussierung durch Kunden und Survey-Firmen gleichermaßen. Dieses Risiko besteht dann, wenn die Kunden in dieser Welt nicht die notwendige methodische Kompetenz für die Dienstleistungswelt besitzen und dadurch lediglich den Preis als Evaluationskriterium für die hier angebotenen Dienstleistungen verwenden, da sie die restlichen Qualitätskriterien nicht sinnvoll zu interpretieren im Stande sind.

Wie dargelegt, bildet die epistemologische Ausgangslage dieser Welt das Wissensbedürfnis von Kunden. Im Gegensatz zur Beratungswelt stellt dies eine Artikulationsfähigkeit in Bezug auf ein organisationales Problem voraus. Auf der 
Basis einer Wissenslücke wird durch den Kunden eine Fragestellung artikuliert. ${ }^{37}$ Diese Fragestellung kann jedoch nicht unabhängig vom Marktangebot betrachtet werden. Denn im Gegensatz zur Informationswelt und zur akademischen Welt findet keine individualisierte Auftragsvergabe statt, sondern ein Einkauf von vordefinierten Dienstleistungen, welche auf einem anonymen Markt angeboten werden. Die Fragestellung muss folglich mit den verfügbaren Erhebungsmethoden und -strategien abgeglichen werden, um in dieser Welt tatsächlich umsetzbar zu sein. Das Marktangebot ist dabei nicht unabhängig von der Marktstruktur, d. h. der Anzahl und der Struktur der verschiedenen Survey-Firmen. Einen Einfluss auf das Marktangebot hat außerdem die Standardisierungsmöglichkeit der angebotenen Dienstleistung, d. h. inwiefern sich die Dienstleistung überhaupt als standardisierte Dienstleistung anbieten lässt. Zudem wird das Marktangebot durch die (Markt-)Nachfrage nach den verschiedenen Dienstleistungen bestimmt. Im Hinblick auf eine idealtypische Distributed Cognition lässt sich die Epistemologie dieser Welt wie in Abb. 5.5 zu sehen darstellen.

Deutlich wird aufgrund von Abb. 5.8, dass die Festlegung der Fragestellung in dieser Welt durch die Ko-Konstruktion zwischen einer durch die Auftragnehmer-Organisation festgestellten Wissenslücke und einem Marktangebot stattfindet. Auf dieser Grundlage wird schlussendlich das spezifische Wissensformat der Dienstleistungswelt, die „Entscheidungsgrundlage“, produziert.

\subsection{Welt der Survey-Information}

Das Wissensformat dieser Survey-Welt ist die Information. Der Begriff der Information wird hier gewählt, um die hohe Reichweite des hier produzierten Wissensformats zu unterstreichen. Folgt man Thévenot, so bedingen Informationen ein hohes Maß an Forminvestitionen, um die hohe Reichweite des Wissens zu erreichen (Thévenot 1997, S. 207 f.). Damit geht unweigerlich das Schaffen einer eigenen - im Hinblick auf Survey-Welten statistischen - Realität einher, welche mit Bezug auf das Regime des Engagements weit vom Vertrauten entfernt ist.

\footnotetext{
${ }^{37}$ Klassischerweise sind dies in dieser Welt Fragen der Vermarktung, insbesondere der Kundenwahrnehmung und -bewertung von Produkten und Dienstleistungen. Dies können jedoch auch Fragen zu „Kunden“ durch öffentliche Institutionen sein. Wie bereits erwähnt liegt die Trennung zwischen Survey-Welten nicht an der Art des Auftraggebers - hier also zwischen privatwirtschaftlichen und öffentlich-rechtlichen Auftraggebern - sondern an dem zu erreichenden Wissensformat eines Surveyprojekts.
} 
Das Wissen in der Informationswelt stellt folglich aus der Perspektive des thévenotschen Informationskonzepts eine Realität sui generis dar, welche mit einer starken Transformation des vertrauten (oder auch lebensweltlichen) Wissens einhergeht. ${ }^{38}$ Informationen basieren folglich auf Äquivalenzkonventionen mit einer hohen Reichweite (Desrosières 2005, S. 13 f.). Thévenot beschreibt eine eigene Rechtfertigungsordnung der Information (Thévenot 1997, S. 233 ff.). Ein zentrales Gütekriterium ist darin die Uniformiertheit von Informationen und nicht nur deren Bekanntheit wie in der Rechtfertigungsordnung der Meinung. Der Qualitätstest besteht in dieser Rechtfertigungsordnung in der Kommunizierbarkeit von Informationen. Damit liegt das Konzept der informationsweltlichen Rechtfertigungsordnung von Thévenot nah am hier beschriebenen Informationskonzept dieser Survey-Welt.

Im Verhältnis zur Welt der Beratung und der Welt der Dienstleistung haben die hier produzierten Daten eine hohe zeitliche wie auch räumliche Reichweite und sind ein Wissensinstrument für ein Feld, einen Markt oder eine Gesellschaft wie beispielsweise Branchenstatistiken oder die öffentliche Statistik. Diese hohe Reichweite basiert auf starken statistischen Formen, ${ }^{39}$ das heißt auf deren hoher gesellschaftlicher Durchdringung. Die hier produzierten Daten müssen deswegen immer als Ko-Produkt mit etablierten statistischen Formen gesehen werden. Statistische Formen basieren auf Forminvestitionen in die Messbarmachung von sozialen Sachverhalten (das heißt in die Operationalisierung). ${ }^{40}$ Das zentrale statistische Konzept stellt hierbei der Indikator dar. Eine zentrale Eigenschaft von Indikatoren sind dessen Eigenheiten der Früherkennung, der Dauerbeobachtung und der Fortschrittskontrolle von sozialen Gegebenheiten (Meyer 2004, S. 6).

\footnotetext{
${ }^{38}$ Und es ist genau diese Realität sui generis, welche Cicourel an der quantitativen Sozialforschung bemängelt, welche aus der Perspektive der EC jedoch auf kollektiv geteilten Konventionen basiert (Diaz-Bone 2018, 331 ff.).

${ }^{39}$ Die Reichweite von numerischen Zahlen basiert folglich nicht allein auf deren numerischen Charakter (Porter 1995, S. preface ix). Die ,gemeinsame Sprache“ (Espeland und Stevens 2008, S. 419) wird also nicht durch die Zahlen selbst, sondern durch die die Zahlen organisierende Formen erreicht. Dies lässt sich gut an den Harmonisierungsbemühungen der öffentlichen Statistik verschiedener europäischer Ländern aufzeigen, in welchen unterschiedliche Konventionen der Messung harmonisiert werden (Brousse 2004, S. 15 ff.). Die Erhöhung der Reichweite wird über eine Harmonisierung der statistischen Formen erreicht.

${ }^{40}$ Eingewendet werden kann hier, dass sämtliche Erhebungen auf einer statistischen Form beruhen, indem operationalisiert werden muss, wie ein sozialer Sachverhalt gemessen werden soll. Dem kann nichts hinzugefügt werden. Spezifisch für die Informationswelt ist aber die notwendige hohe Reichweite der in dieser Welt verwendeten statistischen Formen.
} 
Dies setzt eine dauerhafte Messung voraus, da sämtliche Eigenheiten auf dem Vergleich zu vor- oder nachlaufenden Messungen aufbauen. Beispiele für statistische Formen sind die Sinus Milieus (SINUS Markt- und Sozialforschung GmbH 2017), WEMF-Studien (WEMF AG für Werbemedienforschung 2017), Absolventenbefragungen oder auf Umfragen aufbauende Konjunkturbarometer.

Die hier gewonnenen Daten dienen der Lokalisierung der organisationalen oder staatlichen Position. Konjunkturbarometer zeigen die Wirtschaftslage an und Sinus-Milieus geben Informationen über die eigenen Kunden. Es kann folglich argumentiert werden, dass sich die Studien von Desrosières zu statistischen Regimen maßgeblich auf die Informationswelt beziehen, da hier einerseits die öffentliche Statistik der Untersuchungsgegenstand war, andererseits die Vergleichbarkeit als Kernpunkt der Quantifizierung herausgearbeitet wurde (Desrosières 2009a, S. 312). Zentral für die Informationswerdung ist folglich die Vergleichbarkeit, was die Voraussetzung ist für die Schaffung eines für sämtliche involvierten Akteure geltenden Äquivalenzraumes und die anfangs thematisierte hohe zeitliche und räumliche Reichweiter der in dieser Welt produzierten Daten erst ermöglicht (Desrosières 2005, S. 361). Das Streben nach Vergleichbarkeit in der Informationswelt zeigt sich auch in der methodischen Praxis. Im folgenden Zitat verweist ein akademischer Survey-Forscher auf die Unterschiede zwischen der akademischen Welt und der Informationswelt im Hinblick auf unterschiedliche Organisationskulturen von Survey-Firmen:

Nicht immer aber [...] aber oft und [...] ich glaube eben, dadurch dass sie sehr viel für [die öffentliche Statistik] arbeiten, äh, haben sie sich angewöhnt und [die öffentliche Statistik] funktioniert eben so äh, dass man, dass man, die Dinge bis ins letzte Detail fixiert. Das kann man in der Statistik auch besser machen, weil die wirklich routinemäßig, also die sind froh, wenn sie nicht innovieren, sondern, das gleiche machen wie das letzte Mal, oder? Das ist ein großer kultureller Unterschied zwischen Statistik und und wissenschaftlicher Forschung und und und dafür setzen sie den Rahmen ganz klar, ganz klar fest und ich hab manchmal das Gefühl, [Firma X] hat sich sehr stark daran, daran gewöhnt oder?

Der Interviewpartner macht hier den starken Fokus auf eine Vergleichbarkeit der Erhebung durch die öffentliche Statistik - welche der Informationswelt zugerechnet werden kann - deutlich, welche umgekehrt für die akademische Survey-Forschung ein mangelndes $\mathrm{Maß}$ an Flexibilität bedeutet. Zusätzlich zu der bereits in der Dienstleistungswelt vorfindbaren Vergleichbarkeit innerhalb der Erhebungen einer Survey-Firma, welche durch die Standardisierung ermöglicht wird, findet in der Informationswelt jedoch eine Vereinheitlichung der Erhebung 
über alle sich auf eine bestimmte statistische Form beziehenden Survey-Firmen statt. ${ }^{41}$ Es ist diese Vereinheitlichung, welche als transorganisationale Standardisierung beschrieben werden kann, welche die hohe Reichweite dieser Welt begründet. ${ }^{42}$ So werden beispielsweise Sinus-Milieus nur durch Sinus selbst erhoben. ${ }^{43}$ Denn gerade die Einheitlichkeit macht den Informationswert von Sinus-Milieus aus (Diaz-Bone 2004; SINUS Markt- und Sozialforschung GmbH 2017). ${ }^{44}$ Die für diese Welt spezifische Qualität der Erhebung hängt hier deswegen stark von der Reichweite der statistischen Form ab.

In dieser Welt gibt es eine Trennung in staatliche und privatwirtschaftliche Auftraggeber und Datenanbieter. Dies macht für die hier dargestellte produktionsweltliche Koordinationsform keinen Unterschied, da die Informationsfunktion und die Produktion der Daten auf der Basis von statistischen Formen mit einer hohen Reichweite für beide Statistikproduzenten gelten. Unterschiede zeigen sich jedoch in der Entstehung und der Verwaltung der statistischen Formen. So ist das Erstellen von Indikatoren eine eminent politische Angelegenheit ${ }^{45}$ (Bhuta et al. 2018; Salais 2007, 2010; Bruno et al. 2014; Rottenburg et al. 2015). Politisch ist diese Angelegenheit durch das hier notwendige hohe Maß an Allgemeinheit. Tatsächlich müssen auch privatwirtschaftliche Auftraggeber ,politische“ Arbeit leisten. Sie müssen in für einen Bereich möglichst allgemeingültige Formen investieren. Die Form muss - unabhängig ob staatlich oder privatwirtschaftlich

\footnotetext{
${ }^{41}$ Storper und Salais sprechen in Bezug auf die industrielle Produktionswelt von allgemeinen industriellen Normen, welche die Vergleichbarkeit der durch verschiedene Produzenten gefertigte Produkte gewährleisten (Storper und Salais 1997, S. 21). Auch diese Normen basieren auf Forminvestitionen.

${ }^{42}$ Es ist diese Vergleichbarkeit, welche zu der von Ezrahi beschriebenen „Flachheit“ von Informationen führt (Ezrahi 2004, S. 257).

${ }^{43}$ Bzw. in Lizenz durch andere Survey-Firmen wie beispielsweise MIS Trend in der Schweiz.

${ }^{44}$ Und es ist diese Einheitlichkeit, welche dann in einem nächsten Schritt das Benchmarking ermöglicht auf der Basis von einheitlichen statistischen Formen. Vgl. hierzu auch Desrosières (2000, S. 178).

${ }^{45}$ Dies zeigt sich einerseits bei den Grundlagen der Sozialindikatorenforschung: „In der Funktion von Wohlfahrtsmaßen haben soziale Indikatoren immer auch einen unmittelbaren normativen Bezug, indem sie Unterschiede oder Veränderungstendenzen der Lebensverhältnisse anzeigen, die als besser oder schlechter bewertet werden können“ (Noll 2003, S. 449 f.). Noch direkter zeigt sich diese Notwendigkeit in der öffentlichen Statistik, da die hier produzierten Daten grundsätzlich für eine gesamte nationale Gesellschaft relevant sein sollen. Hierzu werden eigene Prozesse definiert, um der politischen Dimension von Indikatoren gerecht zu werden (LUSTAT Statistik Luzern 2012).
} 
- zu einer „Währung“46 im betreffenden Markt oder sozialen Feld werden, auf welche die verschiedenen Akteure setzen und diese als legitime Form für einen bestimmten sozialen Sachverhalt anerkennen. ${ }^{47}$ Dies führt zu einem wahrgenommenen starken Realismus der hier produzierten Daten, bzw. eine Naturalisierung der hier verwendeten statistischen Formen. Nichtsdestotrotz basieren diese Daten auf (starken) Forminvestitionen. Dies hat die von Desrosières beschriebene Kluft zwischen der konventionellen Produktion und der Nachfrage nach realen Daten zu Folge (Desrosières 2009a). ${ }^{48}$

Regimetheoretisch betrachtet basiert die Informationswelt folglich stark auf dem Rechtfertigungsregime. An die hier erhobenen Kategorien wird ein hoher Anspruch im Hinblick auf deren Verallgemeinerbarkeit und breite gesellschaftliche Akzeptanz gelegt. Statistiken müssen folglich durch einen politischen Prozess auf ein rechtfertigbares Niveau gehoben werden. ${ }^{49}$ Wie bereits dargestellt, gilt dies nicht nur für die öffentliche Statistik, sondern in einem gewissen Maß auch für private Anbieter von survey-basierten Informationen. ${ }^{50}$ Denn auch hier wird eine höhere Form der Allgemeinheit von Erhebungskategorien angestrebt als beispielsweise in Erhebungen der Dienstleistungswelt, deren Relevanzrahmen auf die organisationalen Grenzen beschränkt ist. Am stärksten gilt die Notwendigkeit einer hohen Reichweite der Kategorien natürlich für nationale oder transnationale Statistiken, da hier das öffentliche Interesse direkt tangiert wird. Durch die Kollektivität der Survey-Produktion in der Informationswelt ist die

\footnotetext{
${ }^{46}$ Die WEMF-Werbemittelforschung spricht von den MACH-Studien treffend als einer Währung für den Werbemittelmarkt (Jost und Custer 2013, S. 2).

${ }^{47}$ Ein Beispiel, an welchem sich die Konkurrenz von statistischen Formen gut aufzeigen lässt, ist im Schweizer Surveymarkt das Verhältnis des „Psychologie-Radars“, welches von Demoscope entwickelt und als Grundlage für Lebensstil-Gruppierungen dient (Demoscope AG), zu den Sinus-Milieus, welche durch das deutsche Sinus-Institut entwickelt wurde und in der Schweiz unter Lizenz bei M.I.S. Trend zur Lebensstil-Gruppierung verwendet wird (M.I.S Trend S.A). Beide statistischen Formen verfolgen einen ähnlichen Zweck, konnten sich jedoch nicht als „Währung“ durchsetzen und sind folglich in ihrer Reichweite, d. h. beispielsweise für einen Vergleich zwischen Firmen, auf eine Survey-Firma beschränkt.

${ }^{48}$ Dies ist auch in den anderen Survey-Welten der Fall, jedoch in der Informationswelt ist die Kluft besonders stark infolge der naturalisierten statistischen Formen.

${ }^{49}$ Dies sollte jedoch nicht mit dem Fehlen von Kritik verwechselt werden. Kritik und darauf folgende Rechtfertigung ist vielmehr charakteristisch für dieses Regime (Boltanski und Thévenot 2007, S. 287 ff.).

${ }^{50}$ Jedoch oft in einem geringeren Masse als bei der öffentlichen Statistik, weil sich diese stärker als beispielsweise staatliche Statistiken in Richtung eines Kompromisses mit der Dienstleistungswelt hin bewegen.
} 
staatsbürgerliche Rechtfertigungsordnung eine zentrale Koordinationsform im Hinblick auf die Erstellung von Kategorien (Boltanski und Thévenot 2007, S. 254 ff.). ${ }^{51}$ Dies gilt wiederum am stärksten für Erhebungen der öffentlichen Statistik. Denn privatwirtschaftliche Erhebungen, welche der Informationswelt zugerechnet werden können, müssen im Hinblick auf die Finanzierung oft einen Kompromiss mit der marktweltlichen Rechtfertigungsordnung und dadurch mit dem kollektiven Ziel der Erhebung eingehen. Der Allgemeinheitsanspruch dieser Welt muss auch im Erhebungsprozess beachtet werden. Dies wird im folgenden Zitat erkennbar, in welchem ein Mitarbeitender einer staatlichen Statistikstelle auf die spezifische Qualität der dort erstellten Statistiken eingeht und dabei auf den unterschiedlichen Charakter von staatlichen Umfragen zu anderen Umfragen verweist. ${ }^{52}$ Eine zentrale Qualität der staatlichen Umfragen stellt die Transparenz des Vorgehens gegenüber den Datennutzenden dar, wie ein Mitarbeiter der öffentlichen Statistik deutlich macht:

[...] sie sagen was und wie die Qualität ist und äh das kostet dann häufig mehr Geld, wird dann manchmal nicht überall gleich verstanden, dass eine Umfrage bei uns mehr kostet als äh, wenn sie eine quick and dirty Umfrage machen in einem Panel dann sind wir bei 2000.- aber können nicht, und da ist beispielsweise auch ein Qualitätselement, dass wir aufzeigen, wie wir das gemacht haben, dass sie ganz viele privatwirtschaftliche Aufträge gar nicht haben [...].

Die Transparenz ist auch eine von der akademischen Welt genannte Qualität von Umfragen. Durch die generische Ausrichtung beider Welten soll ein transparentes Vorgehen eine öffentliche (bzw. im Fall der akademischen Welt eine wissenschaftsöffentliche) Kritik und Rechtfertigung über den Stellenwert der Daten ermöglichen.

Die Informationswelt ist oft durch eine dreiteilige statistische Kette gekennzeichnet, welche sich auf Survey-Firmen, Datenkoordinatoren und Datennutzende verteilt. Dies ist dadurch begründet, dass die hier zentrale hohe Reichweite der Daten oft durch Datenkoordinatoren wie statistische Ämter, Informationsinter-

\footnotetext{
${ }^{51}$ Die Entwicklung von der ,amtlichen“ hin zur „öffentlichen“ Statistik kann als Wandel von der industriellen hin zu einer stärker auf der staatsbürgerlichen Rechtfertigungsordnung basierenden Koordinationsform interpretiert werden (Diaz-Bone 2010a). Dies entspricht einem Wandel von der Statistik als primär einem Steuerungsinstrument hin $\mathrm{zu}$ einer Statistik, welche auch das Ziel einer öffentlichen und allgemein zugänglichen Informationsbereitstellung verfolgt.

${ }^{52}$ Die Kritik scheint sich insbesondere auf Umfragen der Dienstleistungswelt zu beziehen.
} 
mediäre, Vereine etc. organisiert wird. Bei zweiteiligen Quality-Chains wird die Formgebung direkt durch die Survey-Firma übernommen, bzw. die Datenerhebung direkt durch statistische Ämter etc. durchgeführt. Datenproduktion findet hier - in starkem Gegensatz zur Beratungswelt - stark distribuiert und oft auf verschiedene Organisationen verteilt statt. Die auftraggebenden Organisationen sind hier folglich nicht die eigentlichen Datennutzer. Vielmehr sind sie oft formgebende und produktvermarktende Organisationen, welche lediglich die Befragung in Auftrag geben. Hier ist die wirtschaftliche Beziehung zwischen Kunden- und Survey-Firma deswegen primär durch die industrielle Rechtfertigungsordnung charakterisierbar, welche mittels eines umfassenden Katalogs an Erhebungsvorgaben die Qualität gewährleisten will. Dieser Qualitätskatalog hat seinen Ursprung dabei nicht auf der Seite der Survey-Firmen, bzw. basiert nicht wie in der Dienstleistungswelt auf allgemeinen, wenigen Qualitätsindikatoren des Survey-Marktes, sondern wird durch die Kundenorganisation definiert. Die Kundenorganisation greift hier folglich relativ direkt auf die Infrastruktur der Survey-Firma zu, ohne den „Umweg“ über Marktangebote. Zentral und ein Unterschied zur Dienstleistungswelt - ist auch die direkte Kontrolle und das intensive Reporting des Erhebungsprozesses, wie ein Mitarbeitender bei der öffentlichen Statistik deutlich macht:

Also wir erwarten natürlich dass die Leute relativ genau uns äh, rapportieren, was sie wann wie machen, wir gehen da auch hin, kontrollieren das, da nimmt man auch die Verantwortung der Qualität nicht ab, oder? Nein, es ist natürlich, die Leute, die bei uns in diesem Bereich tätig sind, die sind selber recht stark äh Fachleute, oder?

Während die Kundenorganisation in der Dienstleistungswelt ein Produkt einkauft, ist es hier mehr das Verfügen über die Infrastruktur, welche eingekauft wird. Im Gegensatz zur Beratungs- und Dienstleistungswelt findet hier eine Verschiebung des Experten-Status statt. Es ist in dieser Welt folglich die Kundenorganisation, welche die Kontrolle über den Produktionsprozess übernimmt. Dies wird im nächsten Zitat des Mitarbeitenden bei der amtlichen Statistik deutlich, bei welcher er die Arbeitsaufteilung der Beratungs- und Dienstleistungswelt kritisiert:

[...] Also ich glaube sie dürfen als Arbeitgeber die Verantwortung über die Qualität nicht abgeben, quasi. Wirklich wirklich simpel und das passiert natürlich in der Privatwirtschaft natürlich relativ schnell, weil man ist sich gewohnt, das ist das Expertenwissen quasi eben, extern einzukaufen und sie gehen wieder davon aus, ich komme ja deswegen zu ihnen, weil sie es besser wissen [...]. 
Die Umkehrung des Expertenstatus im Vergleich zur Dienstleistungswelt liegt darin begründet, dass das Wissensformat der Information durch Datenkoordinatoren gewährleistet wird, welche in dreiteiligen Quality -Chains die Auftraggeberseite darstellt.

Datennnutzende sehen sich in dieser Welt mit einem überschaubaren Markt konfrontiert. Die Vereinheitlichung der hier produzierten Daten bedingt, dass sich kaum viele unterschiedliche Datenproduzenten oder -koordinatoren auf einem Markt halten können. Durch die Eigenschaft der hier produzierten Daten als kollektive Orientierungsquelle in vielerlei Belangen ist es zudem oft so, dass die Daten durch staatliche Stellen oder gemeinnützige Institutionen produziert werden und somit gar kein Markt besteht. Charakteristisch ist dabei aus Sicht der Datennutzenden der Produktcharakter der Befragungsdaten (vgl. hierzu auch Abschn. 7.2). Infolge der generischen und standardisierten Ausrichtung dieser Welt werden hier standardisierte Daten ohne Rücksicht auf einzelne Datennutzende erhoben. Der Datennutzende sieht sich folglich mit vorerhobenen Daten konfrontiert.

Ein typisches Produkt dieser Survey-Welt sind amtliche Statistiken ${ }^{53}$ oder auf statistischen Erhebungen wie den WEMF-Studien oder Sinus-Milieus aufbauende Erhebungen. Durch die generische Natur der produzierten Survey-Daten funktioniert der primäre Wettbewerb zwischen den Survey-Firmen um Transparenz, Genauigkeit und Einheitlichkeit. In diesem Punkt ist die Informationswelt mit der akademischen Welt vergleichbar. Durch die zentrale Rolle der Vereinheitlichung zwischen verschiedenen Erhebungen findet in dieser Welt oft eine langjährige Zusammenarbeit statt. Dies ist auch bei staatlichen Erhebungen der Fall, wird hier jedoch teilweise durch Vorgaben zur regelmäßigen öffentlichen Ausschreibungen von Survey-Aufträgen unterbunden.

Die Kompetenzen der Projektleitenden liegen hier in der genauen Umsetzung von Vorgaben der Auftraggeber auf der Basis der Infrastruktur der Survey-Firma. Zudem müssen sie ein detailliertes Reporting des Erhebungsprozesses gewährleisten. Bei integrierten Organisationen, das heißt Survey-Firmen, welche auch die Rolle eines Datenkoordinators übernehmen, ist es zentral, dass Projektleitende einen Überblick über soziale Felder oder Märkte haben, um die statistische Form aktuell und präsent halten zu können. Es gilt hier, den Bedarf nach

\footnotetext{
${ }^{53}$ Deren Erhebung in der Schweiz durch das Bundesamt für Statistik an Survey-Firmen ausgelagert werden.
} 
(generischer) statistischer Klärung abzuschätzen und daraus statistische Formen zu entwerfen und sie als kollektive Orientierung zu etablieren.

Ein besonderes Verhältnis hat die Informationswelt zur akademischen Welt. Durch die Produktion von Entdeckungen der akademischen Welt kann die Informationswelt nicht ohne einen Bezug auf die in der akademischen Welt gemachten Entdeckungen arbeiten, bzw. riskiert dann, statistische Formen nicht mehr auf dem aktuellen Stand der akademischen Forschung zu halten. Gleichzeitig kann die Informationswelt aber auch nicht auf jede Entdeckung Bezug nehmen, da sie sonst durch ständig neue statistische Formen ihren Kernaspekt der Produktion von allgemeinverständlichen Informationen durch fehlende Reichweite nicht mehr wahrnehmen könnte. Die Informationswelt wird zudem durch die Vielfältigkeit der akademischen Welt im Hinblick auf Theorien und Forschungstraditionen herausgefordert. Eine solche Vielfalt ist jedoch in der Informationswelt nicht möglich durch das notwendig hohe Maß an Allgemeinverständlichkeit und Einheitlichkeit. Sie befindet sich dadurch im Zwiespalt, sich auf akademische Forschungsresultate beziehen zu müssen, aber gleichzeitig eine eindeutige Position daraus formulieren zu müssen. Dies zeigt sich in folgendem Zitat. Für die Sozialberichterstattung im Hinblick auf das Konzept „Lebensqualität" müssen Indikatoren erstellt werden. Dafür wird auf akademische Diskussionen dazu verwiesen:

Neuere Übersichtsarbeiten weisen darauf hin, dass die Diskussion um die differentielle Wirkung von Top-down- oder Bottom-up-Faktoren noch offen ist. Es spricht vieles dafür, dass kombinierte integrative Ansätze zielführender sind als simple Topdown/Bottom-up-Annahmen. Die folgenden drei Theoriestränge begründen die kognitive, hedonische und eudämonische Dimension des subjektiven Wohlbefindens. Dominierend in der aktuellen Wohlbefindensforschung verschiedenster Disziplinen sind hedonische sowie eudämonische Ansätze, die beide ihren Ursprung in der Philosophie der Antike haben (Ehrler et al. 2016, S. 25).

Deutlich wird hier, dass die Informationswelt im Hinblick auf die Investition in statistische Formen mit einer Vielfalt an statistischen Konzepten zur Messung des Wohlbefindens der akademischen Welt konfrontiert wird, welche für die Informationswelt jedoch so nicht umsetzbar ist. Folglich muss nach einem Kriterium der Einschränkung gesucht werden, welches hier auf der Wahl der etablierteren Ansätze beruht. Aus survey-weltlicher Sicht darf deswegen der Forschungsbegriff nicht einseitig auf die akademische Survey-Welt bezogen werden, da sämtliche Survey-Welten Forschung betreiben, jedoch mit unterschiedlichen Wissenszielen. Weischer differenziert zwischen einem „Theoriebezug“ und einem „Anwendungsbezug“: 
So lassen sich die Ziele empirischer Forschung auf einem Kontinuum zwischen den Polen» Theoriebezug «(Entwicklung eines soziologischen Theoriegebäudes) und»Anwendungsbezug «(Diagnosen und Problemlösungen im ökonomischen, sozialen, politischen Feld) verorten (Weischer 2009, S. 19).

Diese Differenz lässt sich auf unterschiedliche Forschungs-Ausrichtungen in der akademischen und informationsweltlichen Survey-Welt beziehen. Die akademische Survey-Welt sucht nach neuen Gegenstands-Konzeptualisierungen durch das Stellen neuartiger Forschungsfragen, welche von Weischer als „Theoriebezug“ beschrieben werden, während die Informationswelt einen stärkeren „Anwendungsbezug" durch die Bezugnahme auf etablierte Theorien herstellt. Entscheidend ist nun, dass beide Orientierungen einen Bestandteil wissenschaftlicher Forschung darstellen und auf Surveys basierende Wissenschaft folglich nicht per se der akademischen Survey-Welt zugeteilt werden können.

In der folgenden Darstellung der (idealtypischen) statistischen Kette der Informationswelt sind einige beschriebene Eigenschaften dieser Welt deutlich zu sehen. Datennutzende (als spätere Kunden) sind als Individuen bei der Konzeption und Durchführung der Befragung irrelevant. Den zentralen Ausgangspunkt der Befragung stellen stattdessen Investitionen in Formen dar, welche die Grundlage für die Indikatorentwicklung darstellen auf Basis einer Koordination mit Forschung aus der akademischen Welt. Die Koordination mit der Survey-Firma (falls es sich nicht um eine integrierte Organisation handelt) findet auf Basis der industriellen Rechtfertigungsordnung via Kontrollen, Tests und einem ausformulierten Pflichtenheft statt. Der Datennutzende schlussendlich sieht sich mit fertigen Daten (bzw. Produkten) konfrontiert, welche er auf seine individuellen Umstände beziehen muss. Diese charakteristische Eigenschaft wird auch in der folgend dargestellten idealtypischen Quality-Chain in Abb. 5.6 deutlich.

Eine Herausforderung stellt in dieser Welt die zunehmende Transnationalisierung der Informationsproduktion und -verteilung dar. Zu nennen sind hier einerseits statistische Vereinheitlichungsbemühungen durch suprastaatliche Einrichtungen wie beispielsweise die Europäische Union oder die Organisation für wirtschaftliche Zusammenarbeit und Entwicklung. Infolge einer global operierenden Ökonomie betrifft dies jedoch auch privatwirtschaftliche Statistiken. Die Informationswelt steht hier vor der Herausforderung einer Vereinheitlichung der nationalen Erhebungen (Brousse 2004; Desrosières 1992), um (erneut) wieder eine (gesamt-)gesellschaftsweite Reichweite der hier produzierten Daten erreichen zu können.

Surveys dienen in der Informationswelt wie dargestellt der Produktion von (Fach-) Öffentlichen Informationen. Nebst der eigentlichen Wissensproduktion 


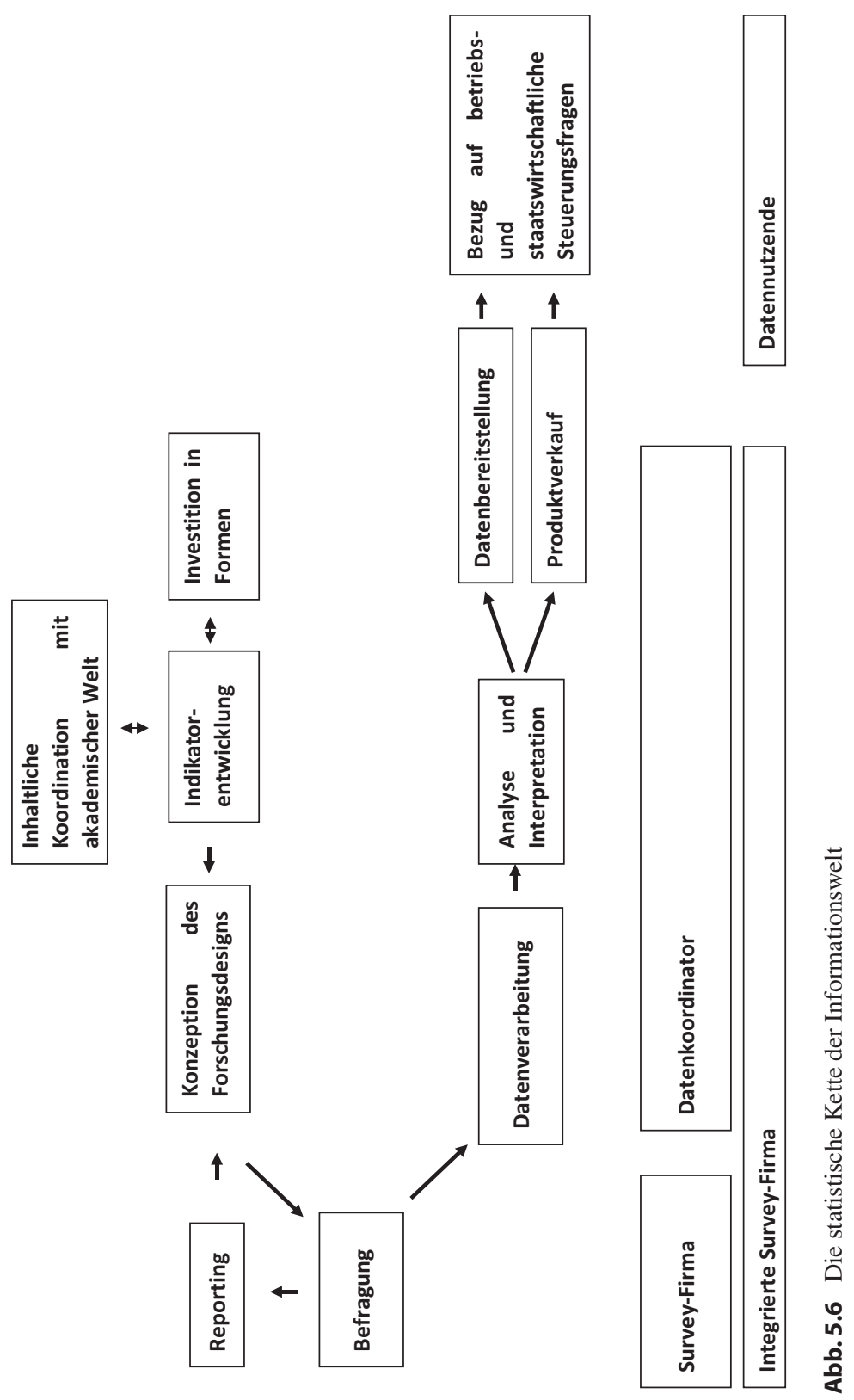


kommt ihnen deshalb auch die Funktion der öffentlichen Repräsentation von Organisationen, Branchen oder gesellschaftsweiten Öffentlichkeiten zu. Surveys sind in dieser Welt folglich nicht lediglich Instrumente der Beobachtung, sondern zusätzlich auch der Repräsentation. Ein Risiko für informationsweltliche Surveys besteht folglich darin, den Fokus der Erhebung alleine auf diese Repräsentationsfunktion zu legen. Konventionentheoretisch betrachtet entspricht dies einem starken Rückgriff auf die meinungsweltliche Rechtfertigungsordnung, welche mit einem Rückgang der industriellen Rechtfertigungsordnung als methodische Anleitung der Erhebung verbunden ist (Boltanski und Thévenot 2007, S. 276 ff.). Denn der starke Fokus auf die Repräsentationsfunktion stellt ein Public Management dar, was als Management der öffentlichen Meinung verstanden werden kann. Der Versuch eines Bestehens in der öffentlichen Meinung stellt dabei die meinungsweltliche Dimension einer solchen Survey-Erhebung dar (Boltanski und Thévenot 2007, S. 245 ff.), welche in Konflikt zu einer Erhebung auf der Basis der industriellen Rechtfertigungsordnung steht, welche durch unabhängige Messapparaturen ein Ergebnis erzielen will. Kritik an der Survey-Forschung, welche Surveys lediglich als Marketinginstrument bezeichnen, zielen exakt auf diese rechtfertigungsbasierte Verschiebung der methodologischen Basis der Erhebung von der industriellen hin zur meinungsweltlichen Rechtfertigungsordnung ab. Die Verankerung von Surveys in der industriellen Rechtfertigungsordnung als Methode zur unabhängigen Messung von sozialen Sachverhalten wird in einem solchen Fall dafür gebraucht, eine durch Surveys scheinbar garantierte objektive Resultate für die meinungsweltliche, öffentliche Repräsentation zu gebrauchen. Der alleinige Fokus auf die meinungsweltliche Rechtfertigungsordnung als Problemfall für diese Survey-Welt darf jedoch nicht darüber hinweg täuschen, dass diese Welt notwendigerweise einen Kompromiss schließen muss zwischen der industriellen Rechtfertigungsordnung, in welcher Surveys methodologisch fundiert sind als grundsätzlich objektive Methoden der Sozialforschung und der meinungsweltlichen Konvention, welche die hohe Reichweite der in dieser Welt produzierten Informationen erst ermöglicht. Denn die Bezugnahme auf in der öffentlichen Meinung bereits etablierte Kategorien, welche eine Bezugnahme auf die meinungsweltliche Rechtfertigungsordnung darstellt (Boltanski und Thévenot 2007, S. 245 ff.), ermöglicht erst die hohe (öffentliche) Reichweite der hier produzierten Informationen. Im Gegenzug muss jedoch ein Kompromiss geschlossen werden mit der industriellen Rechtfertigungsordnung, d. h. es müssen beispielsweise Kategorien ausgehend von der öffentlichen Meinung abgeändert und korrigiert werden, um die Wissenschaftlichkeit von Erhebungen in dieser Welt weiterhin gewährleisten zu können. 
Kritik aus dieser Welt richtet sich einerseits an die gewidmeten Welten. Kritisiert wird hier die fehlende Reichweite der dort produzierten Daten, welche durch eine fehlende Vereinheitlichung der erhobenen Kategorien resultiert. Dies betrifft in besonderem Maß die Beratungswelt, da deren Kategorien bei jeder Erhebung neu definiert werden, was eine Vereinheitlichung grundsätzlich ausschließt. Eine Vereinheitlichung ist zwar in der Dienstleistungswelt teilweise gegeben durch das standardisierte Angebot, dennoch unterscheiden sich die erhobenen Daten einerseits durch die verschiedenen Angebote unterschiedlicher Firmen, andererseits durch die verschiedenen (aber gleichwohl standardisierten) Erhebungsmethoden innerhalb von Survey-Firmen. Aus Sicht der Informationswelt fehlt jedoch eine Vereinheitlichung der Erhebung über verschiedenen Organisationen und Erhebungen hinweg, welche eine hohe Vereinheitlichung und Einheitlichkeit der Daten gewährleisten. Kritik wird jedoch auch an der akademischen Welt formuliert, trotz des Zurückgreifens auf deren Konzepte und Theorien. Die Kritik richtet sich hier ebenfalls an die fehlende Vereinheitlichung der Daten. Diese wird verunmöglicht, da die Orientierung der akademischen Welt am wissenschaftlichen Diskurs eine Einheitlichkeit durch die Notwendigkeit des Neuartigkeitscharakters eine Vereinheitlichung zwischen verschiedenen Erhebungen nicht erlaubt. Denn dadurch kommen in verschiedenen Erhebungen unterschiedliche Konzepte und Theorien als Erhebungsgrundlage zur Anwendung, welche in unterschiedlichen Erhebungskategorien münden, was im Endeffekt die Analyse von Unterschieden zwischen verschiedenen Erhebungen nicht möglich macht.

Die epistemologische Grundlage der Forschung bildet in der Informationswelt nicht die Entdeckung von neuem Wissen, sondern die Produktion von Information, wie in diesem Kapitel ausgearbeitet wurde. Informationen stellen dabei Wissenskategorien mit einer hohen Reichweite, das heißt einer Öffentlichkeitswirksamkeit dar. ${ }^{54}$ Diese hohe Reichweite soll mit dem Konzept der statistischen Form ausgedrückt werden. Tatsächlich ließen sich auch die Kategorien in den restlichen Welten als statistische Form bezeichnen. Der Formbegriff basiert jedoch auf dem Konzept der Investition in diese Formen, das heißt im Aufwand, welche für das Erstellen einer Form notwendig ist (Thévenot 1984). Der in dieser Welt für Forminvestitionen in statistische Kategorien notwendige Aufwand ist in der Informationswelt am höchsten, da hier die höchste Reichweite sämtlicher Survey-Welten erreicht werden muss. Eine Fragestellung beinhaltet in dieser Welt die Berücksichtigung des Informationsbedürfnisses der zu informierenden

\footnotetext{
${ }^{54}$ Dies stellt den meinungsweltlichen Aspekt dieser Survey-Welt dar (Boltanski und Thévenot 2007, S. 245 ff.).
} 


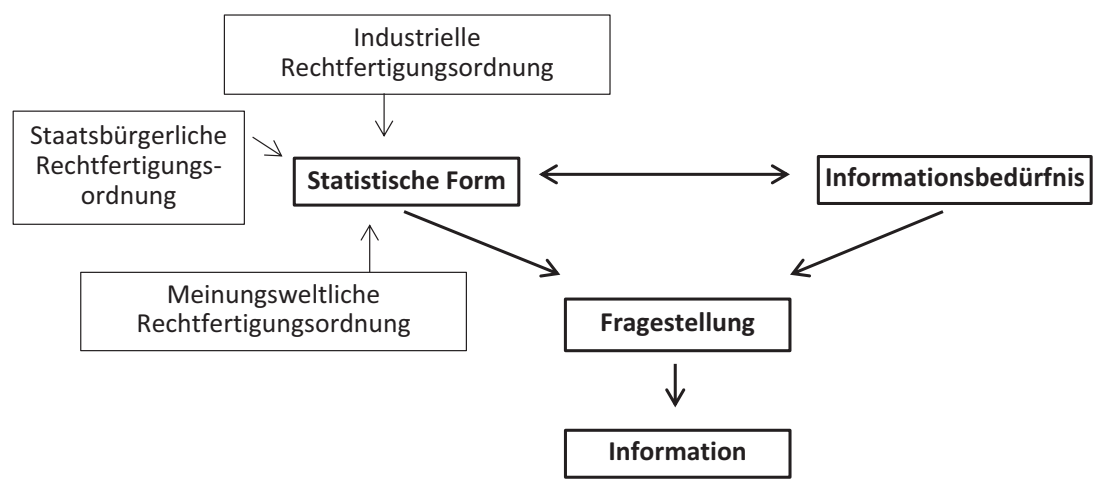

Abb. 5.7 Epistemologie der Informationswelt

Akteure und relevanten statistischen Formen. Der Kompromiss zwischen beiden Aspekten stellt den im letzten Absatz dargestellten Kompromiss zwischen der meinungsweltlichen und der industriellen Rechtfertigungsordnung dar (Boltanski und Thévenot 2007, S. 245 ff., 276 ff.). Insbesondere in staatlichen, aber beispielsweise auch in Erhebungen durch Branchenvereinigungen etc., ist zudem die staatsbürgerliche Rechtfertigungsordnung relevant. In staatlichen Erhebungen findet die staatsbürgerliche Rechtfertigungsordnung durch staatliche Kategorisierungen den Eingang in statistische Formen, in privatwirtschaftlichen Erhebungen ebenfalls durch Kategorien, welche das Kollektiv, beispielsweise einer Branchenvereinigung, beschreiben und repräsentieren. In Abb. 5.7 wird die Epistemologie der Informationswelt zusammengefasst dargestellt.

Mit dieser Grafik soll illustriert werden, dass eine Fragestellung in dieser Welt durch einen Kompromiss zwischen einem spezifischen Informationsbedürfnis und in einer (nationalstaatlichen) Gesellschaft, einer Branche etc., verbreiteten statistischen Formen zustande kommt. Erst diese Ko-Konstruktion zwischen den beiden Elementen ermöglicht die Produktion des Wissensformats der Informationswelt, der „Information“.

\subsection{Akademische Survey-Welt}

Das Wissensformat der akademischen Survey-Welt ist die wissenschaftliche Erkenntnis. Die Erkenntnis bezieht sich hier nicht auf persönliche Erkenntnisse, sondern auf solche mit gesamtgesellschaftlicher Reichweite. Neues Wissen 
soll produziert und alte Gewissheiten infrage gestellt werden (Storper und Salais 1997, S. 36 f.). Die Survey-Qualität bemisst sich hier an der Möglichkeit, neue Erkenntnisse zu generieren. ${ }^{55}$ Zentral ist deswegen der Bezug auf den akademischen Diskurs, um eine Forschungslücke aufzeigen zu können und die Erkenntnis so zu verorten. In Abgrenzung zur Beratungswelt ist es hier der Anspruch, dass die gewonnenen Erkenntnisse nicht nur für einzelne Organisationen relevant sind, sondern gesamtgesellschaftlich neue Erkenntnisse ans Licht bringen. Im Gegensatz zur Informationswelt findet hier keine Orientierung an etablierten statistischen Formen statt, da dies den Neuigkeitsanspruch unterminieren würde. Die verschiedenen Survey-Erhebungen sind deswegen nur bedingt untereinander vergleichbar, da neuartige Fragen beantwortet werden sollen und infolge neuer Theoriegrundlagen neue Forschungsgegenstände untersucht werden. Typische Survey-Projekte für diese Welt sind (singuläre) Erhebungen durch Universitäten und Hochschulen, in denen neuartige Fragestellungen beantwortet werden sollen.

Hier wird auch deutlich, dass sich Panelerhebungen zwischen der akademischen Survey-Welt und der Informationswelt bewegen. Diese können einerseits für die Erhebung von neuartigen Erkenntnissen genutzt werden - beispielsweise durch die Verwendung von Methoden der Kausalanalyse - sie können jedoch auch für ein Monitoring und anschließendes Controlling von sozialen Veränderungen verwendet werden. Der organisatorische und finanzielle Aufwand für Panelstudien zwingt Panel-Forscher jedoch in der Regel dazu, entweder (öffentliche) Geldgeber oder weitere Panel-Forscher zu finden, um den Aufwand für die Panel-Forschung auf mehrere Institutionen verteilen zu können. Die Kollektivität der Forschung zwingt aber andererseits dazu, auch einen gewissen Konsens im Hinblick auf die Erhebung, d. h. insbesondere die befragten Kategorien, auszuhandeln. Es ist dieser Konsens, welcher einen gewissen Kompromiss mit der Informationswelt begründet, ohne dass dadurch jedoch die Panel-Forschung zu einem grundsätzlich informationsweltlichen Instrument würde. Der springende Punkt ist hier lediglich, dass die Panel-Forschung einem steten Konflikt zwischen einer konsensfähigen Mehrheitsmeinung im Hinblick auf die Erhebung und individuellen Erkenntniszielen ausgesetzt ist.

\footnotetext{
${ }^{55}$ Wobei in der akademischen Survey-Welt im Gegensatz zur Produktionswelt der intellektuellen Ressourcen bei Storper und Salais (1997, S. 36 f.) mehrheitlich staatliche Institutionen wie Universitäten und Fachhochschulen aktiv sind und „Erkenntnisse“ produzieren.
} 
Forschende müssen immer in Bewegung sein, um nicht Gefahr zu laufen, repetitive Forschung zu betreiben, welche dann (wenn überhaupt) eher der Informationswelt zuzuordnen wäre und bestätigenden Charakter hätte. In dieser Zwickmühle der Wissensproduktion bewegt sich die Forderung nach Wiederholungsstudien. Für die Produktion von besser abgestütztem Wissen wären solche Wiederholungsstudien notwendig, sie widersprechen jedoch der Logik der Produktion von Erkenntnissen. Hier zeigt sich der ambivalente Charakter der Sozialwissenschaften im Hinblick auf eine Verortung in den verschiedenen Survey-Welten. Einerseits ist sie klar für neue Erkenntnisse zuständig, andererseits wirkt sie auch als Formgeber in die Gesellschaft hinein. Die Forderung nach Wiederholungsstudien bewegt sich genau auf dieser Trennscheide, indem sie fordert, gemachte Erkenntnisse auch abzusichern, zu stützen und so Informationen zu schaffen. Vor diesem Hintergrund ist es folglich verständlich, dass Vertreter der Survey-Welt die Durchführung von Wiederholungsstudien fordern, sich jedoch nur wenige dazu durchringen können. ${ }^{56}$

Die Koordination zwischen Kunden- und Survey-Firma geschieht im Hinblick auf die direkte Steuerung der Erhebung durch die auftraggebende Organisation, vergleichbar zur Informationswelt durch eine Abstimmung im Regime des Plans (Thévenot 2011d, S. 267 f.), das heißt durch das Vorgeben und Überprüfen verschiedener statistischer Parameter, aber auch durch die Vorgabe von organisatorischen Maßnahmen und Prozessen. Die Kundenorganisation will primär auf die Infrastruktur (technisch und personell) der Survey-Firma zugreifen, um das eigene Forschungsvorhaben zu realisieren. Da dem Vorhaben jedoch finanzielle Grenzen gesetzt sind, muss zwischen Kunden- und Survey-Firma stets neu ausgehandelt werden, was möglich ist und was den finanziellen Rahmen sprengen würde. Eine Herausforderung für Survey-Firmen ist dabei stets, die Kundenorganisation als „Organisation in der Organisation“ in den regulären

\footnotetext{
${ }^{56}$ Das Fehlen einer entsprechenden Kultur für Replikationsstudien bemängelt auch ein langjähriger akademischer Survey-Forscher: „Und äh mehrere Replikationsstudien, das ist aber auch ein Problem in der äh in den Curriculum, Curriculä der Forscher, [...] der Wertschätzung von Replikationsstudien dass die eigentlich gar nicht wertgeschätzt werden. [...] Sowohl äh wenn es darum geht ein Papier zu veröffentlichen, [...] als auch dann dann das, das dem potentiellen neuen Arbeitgeber zu verkaufen. [...] Meines Erachtens fehlt da ein bisschen die Kultur. Dass das wertgeschätzt wird [...] mehr Replikationsstudien zu machen“. Dies kann als Kritik aus der Warte der Informationswelt, bzw. als Kritik auf der Basis eines Kompromisses zwischen der Informationswelt und der akademischen Survey-Welt an einer reinen Ausrichtung an der akademischen Survey-Welt verstanden werden, welche Replikationsstudien nicht honoriert, da darin ein Neuigkeitseffekt liegt.
} 
Organisationsablauf integrieren zu können, das heißt dafür zu sorgen, dass auch andere Survey-Projekte reibungslos nebenher abgewickelt werden können.57 Die Anforderungen der akademischen Welt an Survey-Firmen stehen dadurch in einem deutlichen Gegensatz zur Beratungs- und Dienstleistungswelt, wie ein langjähriger Mitarbeiter einer Survey-Firma erklärt:

Jaa, also sie, die Anforderungen sind anders, also die die schreiben dann genau das Prozedere vor und wollen dann zum Schluss Rohdaten. Und die haben dann vollamtliche Projektleiter, die uns die ganze Zeit begleiten. Der [...] klassische Marketingforscher [...] [fragt] wann bekomm ich den Bericht mit den Empfehlungen?

Auch der „Kauf“ der zu erbringenden Dienstleistung gestaltet sich im Vergleich zur Beratungs- und Dienstleistungswelt folglich anders. ${ }^{58} \mathrm{Im}$ Vergleich zur Beratungswelt besteht ein radikaler Gegensatz, da in der akademischen Welt methodische Entscheidungen vollständig durch den Auftraggeber getroffen werden. ${ }^{59} \mathrm{Im}$ Gegensatz zur Dienstleistungswelt sind die Qualitätskriterien für die Survey-Produktion viel umfassender. Die Qualitätskriterien orientieren sich zudem nicht an ,einfachen“, für einen anonymen Markt kommunizierbaren Kriterien, sondern am spezifischen Projekt des Auftraggebers, sowie am sozialwissenschaftlichen Diskurs, welcher methodische Kriterien als Bedingung für die Akzeptanz von Erkenntnissen festlegt. Ein langjähriger akademischer Survey-Forscher setzt sich im folgenden Zitat von der Orientierung der Survey-Forschung in der Dienstleistungswelt an einigen wenigen (marktweltlichen) Qualitätsmassen ab:

[...] und man macht ja immer ein Oversampling, man zieht ja immer mehr, als dass man brauch, aber wir wissen, dass man, wenn man zu viel die Stichprobe zu groß ist, und vor allem wenn man dem Institut freie Hand lässt, dann schmeißen die Adresse um Adresse ins Feld. Die verbrauchen unglaublich viel Adressen, weil

\footnotetext{
${ }^{57}$ Dies hängt natürlich direkt mit der Minderheitenposition der akademischen Welt im Hinblick auf das Auftragsvolumen bei den Survey-Firmen zusammen.

${ }^{58} \mathrm{Zu}$ beachten ist hier, dass Survey-Projekte in den generischen Welten nach der Erfüllung des Auftrages durch die Survey-Firma noch nicht abgeschlossen sind. Für die generischen Welten ist es gerade charakteristisch, dass sie sich an ein Publikum jenseits des Auftraggebers der Survey-Forschung wenden, z. Bsp. an die Scientific Community in der akademischen Welt oder an eine breite Öffentlichkeit wie in der Informationswelt. Dieser Punkt wird weiter unten ausführlicher dargestellt.

${ }^{59}$ Was nicht damit gleichzusetzen ist, dass die Survey-Firma keinen Einfluss auf diese Entscheidungen hat. Absegnen müssen diese Entscheidungen jedoch in der akademischen Survey-Welt immer die Auftraggeber.
} 
das kommt billiger, oder? Das ist aber schlecht für die Stichprobenqualität, die am Schluss dabei rauskommt. Also handeln wir mit denen aus, wie groß die Adresspakete sind, und wann sie diese, wann sie diese, freigeben. Und generell versuchen wir, äh, das Oversampling einzuschränken. Das sind alles diese Qualitäts, Qualitätsanforderungen.

Sichtbar wird hier die unterschiedliche Qualitätslogik der akademischen Survey-Welt in Absetzung zur Dienstleistungswelt. Während sich die Dienstleistungswelt an wenigen Qualitätskriterien orientiert, insbesondere der Anzahl von befragten Personen, orientiert sich die akademische Survey-Welt am wissenschaftlichen Diskurs und muss die daraus entstehenden Qualitätskriterien in einen konkreten Auftrag (im Regime des Plans) auf Basis verschiedener Parameterfestlegungen übersetzten. Survey-Firmen kommt darauf die Aufgabe zu, darzustellen, wie sie diese Qualitätskriterien umsetzen können. ${ }^{60}$ Im Gegensatz zur Dienstleistungswelt findet zudem kein Kauf einer Survey-Dienstleistung statt, welche nach der Erbringung ,abgeholt" wird. Es findet eine intensive Zusammenarbeit ana$\log$ zur Informationswelt statt ${ }^{61}$ und die auftraggebende Organisation nimmt eine starke Kontrolle der Survey-Produktion vor. Dies macht ein akademischer SurveyForscher in Abgrenzung zur Koordinationslogik der Dienstleistungswelt deutlich:

Und wir müssen einfach, äh, schauen, [...] dass unsere Qualitätsansprüche eingelöst werden. Und die Devise ist einfach. Man muss sie, also, wirklich nah begleiten, während der gesamten Befragungsdauer. Also die Idee und die Praxis, die in der Marktforschung zum Beispiel durchaus herrscht, dass man den Auftrag erteilt. Und dort geht ja immer alles ganz schnell, ein paar Wochen später ist dann die Ergebnispräsentation und zwischendurch Blackbox, oder? Das interessiert die Marketingleute auch gar nicht sonderlich. Also bei uns ist es das Gegenteil der Fall, oder? Also wir mischen uns ein in die Rekrutierung der Interviewer, in die Schulung der Interviewer, [...] in die in die in die Feldbegleitung, äh, wir verlangen tägliche Reportings, äh äh, wie wie läuft das, wie sind die [...] wie entwickeln sich die Ausschöpfungs, äh, raten, all diese all diese Dinge und, und sind einfach dauernd in Kontakt mit den [...] Befragungsinstituten, das ist das ist der der Schlüssel, für für die Qualität.

\footnotetext{
${ }^{60}$ So beispielsweise die abnehmende Akzeptanz von Signifikanztests als Beleg für eine neue Erkenntnis (Cumming 2012), bzw. grundlegender die Diskussion um dessen Aussagewert (Harlow 2009).

${ }^{61}$ Unter diesem Blickwinkel ist auch das Angebot einer Survey-Firma zu verstehen, welche die für die Programmierung des Fragebogens zuständigen Informatiker für die entsprechende Zeit direkt zum akademischen Auftraggeber schicken wollte, damit sie die Programmierung in Zusammenarbeit mit dem Auftraggeber direkt vor Ort vornehmen könnten.
} 
Erkennbar wird, dass die Kontrolle der Einhaltung von vorgegebenen Qualitätskriterien in dieser Welt maßgeblich durch den Auftraggeber kontrolliert wird. Dies wird durch die regelmäßige Präsenz des Auftraggebers beispielsweise während telefonischer Befragungen in den Räumlichkeiten der Survey-Firma erreicht, durch ein regelmäßiges Reporting durch die Survey-Firma im Hinblick auf den Befragungsverlauf und durch das Übermitteln von Befragungsdaten wie beispielsweise Keystroke-Files (Olsen und Parkhurst 2013, S. 49). Der eigentliche Befragungsprozess wird so transparent und kritisierbar, wie ein weiterer akademischer Forscher deutlich macht:

\begin{abstract}
Aber wir haben das schon in den Informationen aus der Stichprobe, oder? Da habe ich ihnen mal kurz vorgerechnet, einfach auch um ihnen zu sagen, sie hatten schon die Tendenz zu sagen, ja, ist alles kein Problem, es ist gut gelaufen, alles im Normalbereich, und ja Moment mal, oder? Wir hatten dann probiert, also das ist ja die wie's sein sollte [verweist auf eine Graphik], oder? Und wir haben dann 30 Geschlechtsum, 60 Geschlechtsumwandlungen offenbar. Und wenn man davon ausgeht, dass das nicht Geschlechtsumwandlungen waren innerhalb von ein paar Wochen, dann ist die Wahrscheinlichkeit relativ hoch, dass die Person, das Interview nicht mit der Zielperson geführt wurde, sondern mit einer anderen Person im Haushalt. Also das ist ein klares Indiz, äh, ein klares Indiz, dass da irgendetwas schiefgelaufen ist.
\end{abstract}

Die Notwendigkeit der Begleitung setzt aber auch eine hohe Spezifikation der Ansprüche an die Survey-Produktion voraus. Dies bedingt weiter, dass die akademischen Survey-Forscher nicht nur die Theorie der Survey-Forschung kennen, sondern sich auch in der praktischen Umsetzung von verschiedenen Maßnahmen zur Erhöhung der Datenqualität auseinandersetzen, wie beispielsweise der Erhöhung der Response-Rate etc. Gerade diese Fähigkeit ist eine zentrale Kompetenz von Projektleitenden aufseiten der auftraggebenden Organisation. Im folgenden Zitat kritisiert ein erfahrener Survey-Forscher eine teilweisende ungenügende Praxis diesbezüglich:

[...] the private organizations would do whatever you want, but if you haven't specified it, they won't. And, so I'II see this a lot of times, ahm, people not understand in one, each other's, asking for, and ahm, and they don't specify very well.

Die Kontrolle und Vorgabe des Erhebungsvorgangs kann an einigen (typischen) Stellen mit der spezifischen Kompetenz der Survey-Firmen in Konflikt geraten. Durch die spezifische Arbeitsteilung zwischen Auftraggeber (Planung, Vorgabe von Qualitätskriterien, Datenanalyse) und Survey-Firma (Befragung, Feldplanung) 
ergibt sich ein unterschiedliches Know-how in Bezug auf bestimmte Arbeitsschritte. Die weitgehende Auslagerungspraxis der akademischen Sozialforschung nicht nur in der Schweiz führt zu einer Akkumulation von Praxis-Wissen bei den Survey-Firmen auch für die akademische Survey-Welt (Schnell 2012, S. 370 f.). Ein typischer Konflikt stellt hierbei die Gestaltung des Fragebogens und die Formulierung von Fragen dar. Hier zeigt sich, dass Survey-Projekte in den meisten Fällen Kompromisse zwischen den Koordinationslogiken verschiedener Survey-Welten darstellen. Typisch für Survey-Projekte der akademischen Survey-Welt ist ein Kompromiss mit der Survey-Welt, welcher im Konflikt um die Gestaltung des Fragebogens und die Formulierung von Fragen münden kann. Sind die Auftraggeber stärker an theoretischen Überlegungen orientiert, sehen die Projektleiter der Survey-Firma primär die Begrenzungen in Bezug auf das Frageverständnis durch die Befragten. Ein weiterer Konfliktpunkt stellt die Feldplanung, das heißt die Einsatzplanung von Befragenden, dar, welche grundsätzlich von hoher Bedeutung für das Endergebnis ist, bei welcher den Auftraggebenden jedoch meist die praktische Erfahrung fehlt. Bei beiden genannten Punkten stößt so die Vorgabe von Kriterien durch den Auftraggeber an seine Grenzen, da ihm dazu teilweise die notwendige Erfahrung fehlt.

Im Vergleich zur Informationswelt stellt sich die akademische Survey-Welt im Hinblick auf die Methodenwahl und -anwendung als dynamischer dar. Dies ist dadurch begründet, dass ein zentrales Kriterium für die Datenqualität der Informationswelt die Vergleichbarkeit darstellt, welche in der akademischen Welt hingegen gerade die angestrebte Qualität der Erkenntnis unterminieren würde. Dieser Unterschied zeigt sich bei der Zusammenarbeit mit Survey-Firmen für die akademische Welt anhand fehlender Flexibilität, wie dies im folgenden Zitat deutlich wird: ${ }^{62}$

Nicht immer aber [...] ich glaube eben, dadurch, dass sie sehr viel für das Bundesamt für Statistik arbeiten, äh, haben sie sich angewöhnt und [die amtliche Statistik] funktioniert eben so äh, dass man, dass man, die Dinge bis ins letzte Detail fixiert. Das kann man in der Statistik auch besser machen, weil die wirklich routinemäßig, also die sind froh, wenn sie nicht innovieren, sondern, das gleiche machen wie das letzte Mal, oder? Das ist ein großer kultureller Unterschied zwischen Statistik und und wissenschaftlicher Forschung und [...] dafür setzen sie den Rahmen ganz klar, ganz klar fest und ich hab manchmal das Gefühl, [Survey-Firma X] hat sich sehr stark daran, daran gewöhnt oder?

\footnotetext{
${ }^{62}$ Dabei handelt es sich um dasselbe Zitat, welches bereits zu Beginn des Abschn. 5.4 diskutiert wurde. Es erhält jedoch im Hinblick auf eine Diskussion der akademischen Welt eine eigene Bedeutung.
} 
Typische (Weiter-)Nutzer der hier produzierten Survey-Daten sind die Scientific Community und der Wissenschaftsjournalismus. Hier zeigt sich der Unterschied zur gewidmeten Datenerstellung. Sind die Kunden dort auch die Endnutzer, so geht die Datennutzung im generischen Pol stets über die eigentlichen Survey-Kunden hinaus. Die räumliche Reichweite der hier produzierten Daten ist hoch, da sie stets auf den transnational bis global orientieren wissenschaftlichen Diskurs Bezug nehmen. Allerdings ist der Adressat der hier produzierten Daten primär die sozialwissenschaftliche Forschungsgemeinschaft, sodass präzisiert von einer selektiv hohen räumlichen Reichweite gesprochen werden müsste. Die zeitliche Reichweite ist demgegenüber lediglich mittel. Denn die für die Erhebung verwendeten Kategorien und Theorien sind einem steten Wandel unterworfen, was durch die Produktionsnotwendigkeit neuer Entdeckungen bedingt ist. Zeitlich sind die hier erhobenen Daten folglich einem steten Wandel unterworfen. Die Kompetenzen der Endnutzer im Hinblick auf das Durchführen von Survey-Projekten, das heißt der Scientific Community oder des Wissenschaftsjournalismus, müssen entsprechend hoch sein, da die Dateninterpretation Kenntnis des akademischen Diskurses (sowie deren methodische Diskussion) voraussetzen. Ohne solches Vorwissen ist keine sinnvolle Einordnung und Interpretation der Daten möglich.

Die Kompetenzen der Projektleitenden von Survey-Firmen liegen in der akademischen Welt analog zur Informationswelt im Einhalten des vorgegebenen Qualitätskatalogs. ${ }^{63}$ Die eigentliche Kompetenz des Projektleitenden - das heißt wodurch sich der Projektleitende für den Kunden unverzichtbar machen kann - liegt in der Vermittlung zwischen den Auftragsspezifizierungen des Auftraggebers und den Möglichkeiten der Survey-Firma, d. h. der Praxis der Befragung. Der Investitionsmodus von Projektleitenden besteht deswegen im Einarbeiten in den aktuellen Stand sozialwissenschaftlicher Survey-Methodologie, um eine reibungslose Vermittlung der Aufträge auch in Zukunft gewährleisten zu können. Weiter in der stetigen Verbesserung der auftraggebergemäßen Anwendung der Qualitätskataloge, der Qualitätssicherung und damit einhergehend in der Erhöhung der Transparenz des Befragungsprozesses für die Kundenorganisation.

Da die Finanzierung in dieser Welt nur selten durch die jeweilige Kundenorganisation (bzw. durch die verantwortliche Professur) finanziert wird, sind die Survey-Projekte hier oft durch Projektbeiträge (durch Forschungsfinanzierungs-

\footnotetext{
${ }^{63} \mathrm{Vgl}$. hierzu auch die weiter unten folgenden Ausführungen zum Reporting der Survey-Firmen. Ein erfahrener Projektleiter einer Survey-Firma verweist dabei auf den Umfang des zu leistenden Reportings und die strenge Orientierung bei der Auftragserfüllung an den Vorgaben des Auftraggebers.
} 
einrichtungen wie den SNF etc.) finanziert. Anders als bei der Dienstleistungswelt gilt es hier nicht, eine Balance zwischen einem akzeptablen Preis und einer akzeptablen Qualität zu finden, sondern aus dem vorhandenen Budget eine möglichst hohe Qualität im Sinne des Auftraggebers zu generieren. ${ }^{64}$ Dies verdeutlicht ein akademischer Survey-Forscher im folgenden Zitat:

Und dann ist die Frage, oder? Wir haben jetzt dieses Budget zur Verfügung. Wie holt man da jetzt quantitativ und qualitativ das Beste raus [...]. Und irgendwie die Prozesse so zu definieren und zu steuern, das wir quantitativ und qualitativ das Beste im gegebenen Budget bekommen. Das ist für uns eigentlich die größte Herausforderung, wo wir [...] dauernd daran sind, damit zu überlegen, was machen wir, ist das effizient, kostet uns das zu viel, könnte man das Geld anders einsetzen.

Der akademische Survey-Forscher verweist hier auf die zentrale Bedeutung des Finanzmanagements von akademischen Survey-Projekten, welche durch die Beitrags-Logik des Schweizerischen Nationalfonds begründet ist. Für eine hochstehende Erhebung in dieser Welt ist es folglich notwendig, die finanziellen Ressourcen möglichst umfänglich auszunutzen. Die Schwierigkeit besteht aber gemäß dem akademischen Survey-Forscher gerade darin, diese maximale Ausnutzung im Budget-Rahmen des Projektbeitrages zu halten.

Zentral für das Verständnis der statistischen Kette der akademischen Welt ist der Bezug der Fragestellung wie auch der Ergebnisse auf den akademischen Diskurs, d. h. auf Forschungsresultate zum selben Bereich. Analog zur Informationswelt findet ein intensives Reporting statt, wie ein erfahrener Projektleiter einer Survey-Firma im Gegensatz zur Beratungs- und Dienstleistungswelt hervorhebt:

Wenn ich dann die, die Sozialforscher, die kommen zu uns, die die prüfen genau, wie wir mit jeder Adresse umgehen. Die prüfen wie wir argumentieren, die Prüfen welche Verweigerungsraten wir haben, ääh, die die Schreiben uns vor, dass man jedem Haushalt x-Mal kontaktieren muss um möglichst optimale Ausschöpfung zu erreichen $[\ldots]$.

Deutlich wird hier, wie methodische Entscheidungen durch akademische (und auch informationsweltliche) Auftraggeber nicht aus der Hand gegeben werden

\footnotetext{
${ }^{64}$ In einer Briefing-Sitzung eines akademischen Survey-Projektes hob ein Senior Consultant diese Qualität der Zusammenarbeit hervor. Er wolle demgemäß eine gute Qualität innerhalb des Budget-Rahmens sicherstellen (und eben nicht eine möglichst hohe Qualität ungeachtet der finanziellen Möglichkeiten). Hierin sah er auch ein Differenzierungsmerkmal seiner arbeitgebenden Survey-Firma.
} 


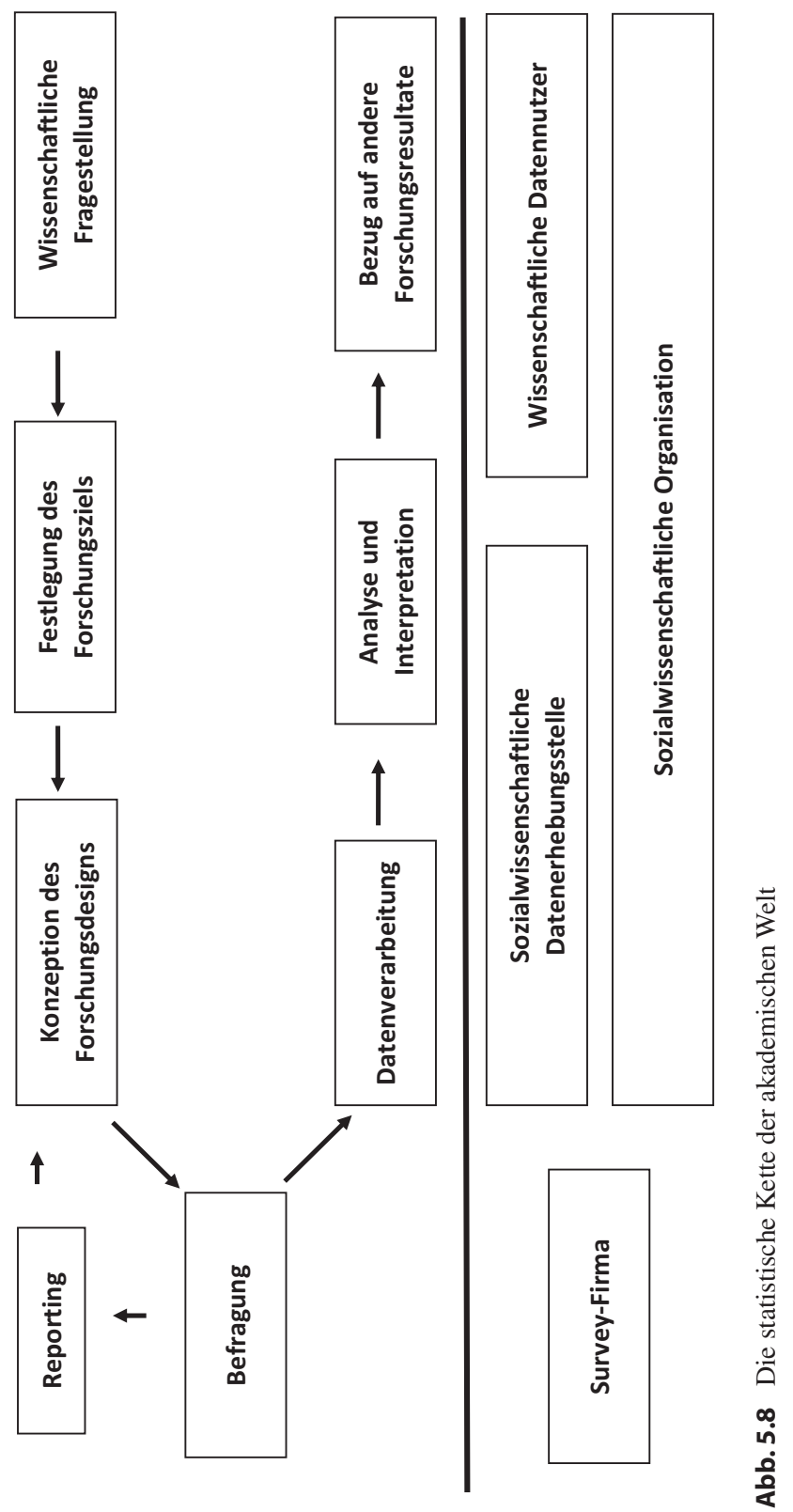


und ein entsprechend intensives Reporting durch die Survey-Firma erfüllt werden muss. Im Vergleich zur Beratungs- und Dienstleistungswelt findet die Konzeption der Forschung grundsätzlich durch den Auftraggeber statt und nicht in Auseinandersetzung mit marktüblichen Qualitätsindikatoren wie in der Dienstleistungswelt, bzw. übertragen an einen Berater. In Abb. 5.8 wird die idealtypische statistische Kette der akademischen Welt dargestellt.

Die zentrale Herausforderung für die akademische Survey-Welt besteht in der Definition der Bedingungen der Erkenntnis. In diesem Zusammenhang ist beispielsweise die zunehmend kritische Diskussion um Signifikanztests als Beleg für eine neue Erkenntnis zu sehen (Cumming 2012, 2014; Harlow 2009). Der zentrale Fokus auf neue Entdeckungen in dieser Survey-Welt führt zu einem Fokus auf die Suche nach neuen Strukturen. Signifikanztests können dabei als minimalster Hinweis auf die Entdeckung von neuartigen Zusammenhängen betrachtet werden. Die Intention einer kritischen Diskussion von Signifikanztests besteht folglich darin, die Schwelle für Entdeckungen zu erhöhen.

Für die Survey-Firmen besteht die größte Herausforderung, die ständig wechselnden Bedürfnisse der Auftraggeber an die eigene Survey-Infrastruktur zu vermitteln. Im Vergleich zur Dienstleistungswelt ist diese Herausforderung in der akademischen Welt stärker. Es findet hier keine Angebotsausrichtung an einem durchschnittlichen und bei der Angebotserstellung anonymen Kunden statt, sondern am individuellen Plan des Auftraggebers. Die Nachfrage in der akademischen Welt lässt sich folglich nicht standardisieren und damit Skaleneffekte erzielen.

Kritik aus der akademischen Welt ist zunächst an die Informationswelt gerichtet. Dies betrifft maßgeblich die Performativität der hier produzierten Zahlen (Savage 2010). Denn anders als im Gebiet der „reinen Wissenschaft“ der akademischen Welt liegt das Ziel in der Informationswelt in der Informationsproduktion und folglich Orientierungsstiftung für eine breite Öffentlichkeit. Diese kollektive Orientierung an den hier produzierten Kennzahlen hat zur Folge, dass soziale Realität nicht nur beschrieben, sondern anhand der den hier getätigten Erhebungen zugrunde liegenden Konzepten auch mitkonstituiert werden (DiazBone 2010b). Daran anschließend wir auch eine Kritik am Konservatismus der Informationswelt von der akademischen Welt aus formuliert. Durch die notwendig breite Abstützung der Kategorien der Informationswelt können die Erhebungskategorien dieser Welt nicht ständig geändert werden, wie dies im Gegenteil dazu in der akademischen Welt gerade den Regelfall darstellt. Damit geht ein Festhalten an Erhebungskategorien einher, welche aus Sicht der akademischen Welt überholt erscheinen und nicht den aktuellen Stand der Forschung abbilden. Die Kritik richtet sich jedoch auch an die gewidmeten Welten. Kern- 


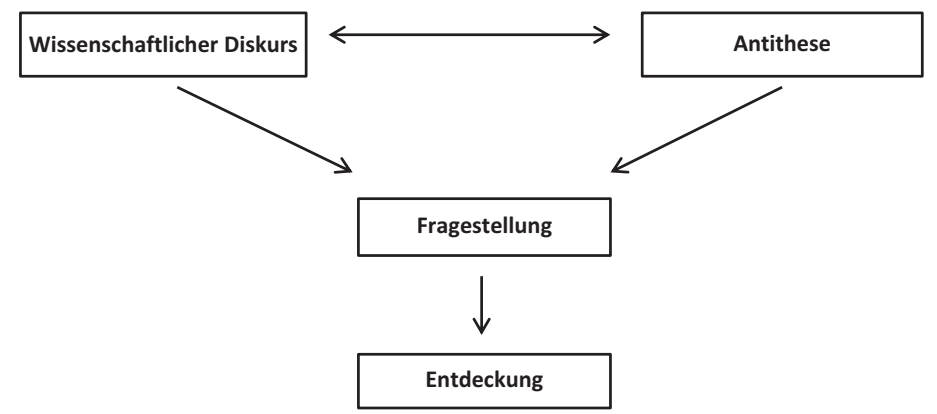

Abb. 5.9 Epistemologie der akademischen Welt

punkt der Kritik ist hier die fehlende Orientierung an einer Wissensproduktion für die Allgemeinheit, das heißt eine Kritik an der fehlenden generischen Ausrichtung der Beratungs- und Dienstleistungswelt.

Die epistemologische Grundlage dieser Welt liegt in der Produktion von Entdeckungen, das heißt neuen allgemeingültigen Einsichten. Neue Entdeckungen werden durch die Absetzung von etablierten Konzepten und Theorien produziert. Es ist die Konterkarierung des wissenschaftlichen Diskurses, welches die Bedingung für neue Einsichten schafft. Dies ist möglich durch die Beforschung von neuen unerforschten Gegenständen - deren Neuheit durch die Absetzung vom aktuellen wissenschaftlichen Forschungsstand koproduziert wird - oder durch das Aufzeigen von Problemen etablierter Theorieansätze. In Abb. 5.9 ist die Epistemologie der akademischen Welt abgebildet.

Deutlich wird, dass sich eine Fragestellung in dieser Welt aus einer Ko-Konstruktion zwischen einem wissenschaftlichen Diskurs und einer Antithese dazu ergibt. Es ist diese Ko-Konstruktion zwischen den beiden Elementen, welche das spezifische Wissensformat dieser Survey-Welt, die ,Entdeckung“, produziert.

\subsection{Survey-Welten im Vergleich}

Das folgende Kapitel dient dem Vergleich der Survey-Welten. Zunächst wird auf die unterschiedlichen Umwelten und Öffentlichkeiten von Survey-Welten eingegangen. Danach wird ein Überblick über die survey-weltliche Verortung verschiedener Produkte vorgenommen. Geklärt wird hier am Beispiel unterschiedlicher Survey-Produkte, inwiefern diese mit verschiedenen Survey-Welten 
korrespondieren. Schlussendlich wird ein vergleichender Überblick über die unterschiedlichen Logiken von Survey-Welten dargestellt.

Bereits in den vorhergehenden Unterkapiteln wurde darauf hingewiesen und dargelegt, inwiefern Survey-Welten nicht nur unterschiedliche Logiken und mentale Prozesse darstellen, sondern eben auch mit unterschiedlich ausgestatteten Umwelten einhergehen. Die EC hat in verschiedenen Analysen immer wieder betont, dass Rechtfertigungsordnungen erst durch eine Ausstattung mit Objekten und Wesen zu einer Welt werden (Boltanski und Thévenot 2007). Es ist folglich diese Verbindung zu Ausstattungen, welche Logiken zu pragmatischen Handlungswelten werden lässt. Zentral ist dabei, wie durch Welten Objekte und Formen spezifisch mobilisiert werden. In seiner Analyse des Marktes für Rechtsprodukte macht beispielsweise Christian Bessy deutlich, wie unterschiedliche Konventionen von Rechtsprodukten systematisch mit unterschiedlichen Organisationsstrukturen und Öffentlichkeiten im Sinne von Rezipienten der Rechtsprodukte einhergehen (Bessy 2012).

Unterschiedliche Umwelten und Öffentlichkeiten lassen sich auch bei den Survey-Welten identifizieren. ${ }^{65}$ So sind Survey-Firmen in der Beratungswelt oft kleine Firmen. Dies hängt maßgeblich mit der schwierigen internen Auftragsweitergabe durch die fehlende Standardisierbarkeit der Aufträge zusammen, sodass Aufträge oft aus „einer Hand“ bearbeitet werden. Auch die Klienten sind hier typischerweise kleine bis mittelgroße Firmen, bei welchen die Einrichtung einer eigenen Forschungsabteilung unverhältnismäßig im Vergleich zur Firmengröße wäre. Es sollte hier aber nicht der Umkehrschluss gezogen werden, dass Klienten in dieser Welt nicht auch große Firmen oder staatliche Stellen darstellen können. Auch dort kann spezifisches Know-how gefragt sein, beispielsweise im Sinne einer Außenperspektive auf die eigenen Strategien und Prozesse. Wissensadressaten sind in dieser Welt organisationsinterne Stellen wie beispielsweise Stabsstellen oder Produktmanager.

Survey-Firmen der Dienstleistungswelt können nur schwierig im Hinblick auf ihre Größe typisiert werden, da diese Welt die zentrale Koordinationsgrundlage des Survey-Marktes darstellt. Dennoch sind Survey-Firmen in dieser Welt typischerweise von einer mittleren Größe. Dies ermöglicht einerseits, die in dieser Welt geforderte Flexibilität von Marktangeboten und Bearbeitungsprozessen sicherzustellen, andererseits durch die Standardisierung und die damit verbundene Möglichkeit von Ansätzen von Economies of Scale auch einen tieferen

\footnotetext{
${ }^{65}$ Storper und Salais beschreiben beispielsweise auch typische Größen von Firmen in den verschiedenen Produktionswelten (Storper und Salais 1997, S. 77 ff.).
} 
Preis im Vergleich zur Beratungswelt zu erzielen (Storper und Salais 1997). Wie im Kapitel zur Dienstleistungswelt dargestellt wurde, ist die in dieser Welt existierende Akteursform des Kunden jedoch an Voraussetzungen im Hinblick auf das Survey-Wissen gekoppelt. Durch diese erhöhten Anforderungen sind es typischerweise mittelgroße bis große Firmen und auch öffentlich-rechtliche Stellen, welche in dieser Welt als Kunden auftreten. ${ }^{66}$ Denn diese sind imstande, interne Stellen zu finanzieren, welche die Übersetzung von internen individuellen Ansprüchen in standardisierte Aufträge wie auch die methodische Kontrolle von Survey-Projekten gewährleisten können. Solche internen Stellen nehmen folglich die Funktion von Intermediären ein, welche gleichzeitig zur Hilfestellung in survey-methodischen Fragen eine Investition in Formen bei internen Aufträgen vornehmen (Diaz-Bone 2018, S. 85 ff.; Thévenot 1984). Wissensadressaten sind in dieser Welt organisationsinterne Stellen wie beispielsweise Stabsstellen oder Produkt Manager.

Survey-Firmen in der Informationswelt sind mittelgroß bis groß. Insbesondere wenn diese zugleich als Formgeber aktiv sind (vgl. Abschn. 5.4), ist eine gewisse Größe unerlässlich, um die mit der Formgebung verbundenen Investitionskosten durch eine hohe Anzahl an Aufträgen wieder auszugleichen. Survey-Firmen, welche in dieser Welt jedoch für Auftraggeber wie staatliche Stellen oder Informationsintermediäre arbeiten, ${ }^{67}$ sind typischerweise eher mittelgroß, um die entsprechende Flexibilität gegenüber dem Auftraggeber gewährleisten zu können. Wie bereits dargestellt, sind Auftraggeber in dieser Welt Informationsintermediäre oder staatliche Stellen, welche sich an einer generischen Informationsproduktion orientieren. Diese orientieren sich - wie auch Survey-Firmen, welche zugleich als

\footnotetext{
${ }^{66}$ Dass auch öffentlich-rechtliche Stellen hier als Kunden dieser Welt aufgeführt sind, soll nicht darüber hinweg täuschen, dass bei solchen Kunden oft ein Kompromiss mit der Informationswelt eingegangen wird. Nichtsdestotrotz können auch Survey-Projekte, welche von öffentlich-rechtlichen Stellen in Auftrag gegeben werden, der Dienstleistungswelt oder auch der Beratungswelt zugeschrieben werden, da schlussendlich nicht die Art des Auftraggebers entscheidend für eine survey-weltliche Zuordnung des Survey-Projekts ist, sondern die dabei zur Anwendung gekommene tatsächliche Logik der Survey-Produktion. Dieser Punkt kann nicht oft genug betont werden, da die Entsubstanzialisierung von Konzepten wie beispielsweise der Organisation in der EC damit direkt zusammenhängt (Diaz-Bone 2009a). Die intuitiv erscheinende Zuordnung beispielsweise von staatlichen Survey-Projekten zur Informationswelt sorgt immer dann für Verwirrung, wenn diese wieder der Erwartung als beratungsweltlich oder Dienstleistungsweltlich koordiniert analysiert und beschrieben werden.
}

${ }^{67}$ Vgl. zu Informations-Intermediären Bessy und Chauvin (2013). 
Formgeber agieren - an einer Nachfrage nach survey-basierter Information noch vor dem Kontakt mit den schlussendlichen Datennutzenden (Storper und Salais 1997). Zentral ist folglich die Investition in Informationsformen, bzw. Erhebungskategorien, durch diese Formgeber, welche das Format einer kollektiven Information erst ermöglichen (Thévenot 2011d). Typische Datennutzende lassen sich in dieser Welt nicht identifizieren, da sich das hier produzierte Wissensformat an der Öffentlichkeit ausrichtet und sich so gleichermaßen an staatliche Stellen, privatwirtschaftliche Firmen und Privatpersonen richtet. Im Hinblick auf Wissensadressaten können unterschiedliche Reichweiten der hier produzierten Informationen identifiziert werden, welche sich insbesondere auch daraus ergeben, wie stark hier ein Kompromiss mit der akademischen Welt oder der Dienstleistungswelt eingegangen wird. Wissensadressaten dieser Welt sind folglich einerseits die allgemeine Öffentlichkeit, bzw. die Gesellschaftsöffentlichkeit und andererseits Branchen- oder Themenöffentlichkeiten.

Survey-Firmen, welche die akademische Survey-Forschung bedienen, sind typischerweise mittelgroß bis groß. Einerseits ist es hier notwendig, eine Flexibilität gegenüber den unstandardisierten Aufträgen gewährleisten zu können, andererseits ist eine gewisse Größe der Survey-Firmen unerlässlich, um die oftmals großen Befragungsmengen bewältigen zu können. Typische Auftraggeber sind in dieser Welt Universitäten und Hochschulen. Daneben ist es jedoch durchaus denkbar, dass auch staatliche Stellen oder Vereine - oftmals in Kooperation mit Universitäten und Hochschulen - akademische Survey-Produkte in Auftrag geben. Zentral ist hierbei die generische Ausrichtung dieser Welt, welche die (gesellschaftliche) Kollektivität von Projekten in dieser Welt verdeutlicht, welche für einzelne Firmen einerseits zu wenig spezifische Resultate hervorbringen und dadurch finanziell nicht rentabel sind. ${ }^{68}$ Typische Datennutzer sind hier einerseits die Wissenschaft. Diese geben die Aufträge einerseits in Auftrag, andererseits besteht eine indirekte Datennutzung in der Wissenschaft darin, weitergehende Analysen und Theorien auf getätigten Erhebungen aufzubauen. Der primäre Wissensadressat von akademischen Erhebungen stellt folglich die Fachöffentlichkeit dar. Zusätzliche Datennutzer stellt der Wissenschaftsjournalismus dar, welcher hier als Intermediär zwischen der Informationswelt und der akademischen

\footnotetext{
${ }^{68}$ Storper und Salais beziehen diese Welt insbesondere auf Hochtechnologiefirmen, aber auch auf Entwicklungsprojekte von Organisationen, welche in der Welt des Marktes oder der Industrie beheimatet sind (1997). Diese scheinen im Hinblick auf die Survey-Forschung kein Äquivalent zu besitzen, bzw. werden hier durch universitäre Forschungseinrichtungen und Hochschulen ersetzt.
} 
Welt fungiert (Bessy und Chauvin 2013; Diaz-Bone 2018, S. 109 ff.). Nutzende sind jedoch generell auch andere Survey-Welten, welche auf die in dieser Welt produzierten Ergebnisse Bezug nehmen und für eigene Zwecke abwandeln. Prominent ist hier die Informationswelt zu nennen, welche einerseits ebenfalls einen hohen Wissenschaftsanspruch verfolgt, für die andererseits die Pluralität der Theorien und Konzepte der akademischen Welt problematisch ist. Ergebnisse der akademischen Welt müssen folglich einer Uniformierung unterzogen werden. In Tab. 5.1 sind die typischen Größen von Survey-Firmen und Käufer-Organisationen sowie die jeweiligen Wissensadressaten der verschiedenen Survey-Welten, zusammengefasst dargestellt.

Die grundlegenden Trennungsprinzipien der verschiedenen Produktionswelten sind bei Storper und Salais die Gegensatzpaare spezialisiert - standardisiert und gewidmet - generisch (Storper und Salais 1997, S. 26 ff.). In den vorhergehenden Darstellungen der Survey-Welten wurde bereits auf die wissensontologische Grundlegung der verschiedenen Welten eingegangen und

Tab. 5.1 Typische Auftragnehmer und Auftraggeber der verschiedenen Survey-Welten

\begin{tabular}{|c|c|}
\hline Beratungswelt & Dienstleistungswelt \\
\hline $\begin{array}{l}\text { Größe der Survey-Firmen: } \\
\text { Klein bis mittelgroß }\end{array}$ & $\begin{array}{l}\text { Größe der Survey-Firmen: } \\
\text { Mittelgroß }\end{array}$ \\
\hline $\begin{array}{l}\text { Typische Klienten: } \\
\text { Kleine bis mittelgroße Firmen }\end{array}$ & $\begin{array}{l}\text { Typische Kunden: } \\
\text { Mittelgroße bis große Firmen }\end{array}$ \\
\hline $\begin{array}{l}\text { Wissensadressaten: } \\
\text { Organisationsinterne Stellen }\end{array}$ & $\begin{array}{l}\text { Wissensadressaten: } \\
\text { Organisationsinterne Stellen }\end{array}$ \\
\hline Akademische Welt & Informationswelt \\
\hline $\begin{array}{l}\text { Größe der Survey-Firmen: } \\
\text { Mittelgroß bis Groß }\end{array}$ & $\begin{array}{l}\text { Größe der Survey-Firmen: } \\
\text { Mittelgroß bis Groß }\end{array}$ \\
\hline $\begin{array}{l}\text { Typische Auftraggeber: } \\
\text { Universitäten, Hochschulen }\end{array}$ & $\begin{array}{l}\text { Typische Auftraggeber: } \\
\text { Öffentliche Statistik, Informationsintermediäre }\end{array}$ \\
\hline $\begin{array}{c}\text { Typische (Daten-)Endnutzer } \\
\text { Wissenschaft, Wissenschaftsjournalismus, } \\
\text { andere Survey-Welten }\end{array}$ & $\begin{array}{c}\text { Typische (Daten-)Endnutzer: } \\
\text { Staatliche Stellen, privatwirtschaftliche Firmen, } \\
\text { Privatpersonen }\end{array}$ \\
\hline $\begin{array}{c}\text { Wissensadressaten: } \\
\text { Fachöffentlichkeit, Öffentlichkeit durch } \\
\text { Intermediäre }\end{array}$ & $\begin{array}{c}\text { Wissensadressaten: } \\
\text { Gesellschaftsöffentlichkeit, Branchen- oder } \\
\text { Themenöffentlichkeiten }\end{array}$ \\
\hline
\end{tabular}


die Unterschiede zwischen ihnen. Ungeklärt bleibt jedoch die Frage, wie die von Storper und Salais dargestellten Gegensatzpaare aus survey-weltlicher Perspektive zu verstehen sind. Dem soll im folgenden Kapitel nachgegangen werden. Der für Sozialwissenschaftler naheliegendste Pol ist der generische Pol. Das Ziel der Survey-Forschung liegt hier in der Produktion von gesellschaftlichem Wissen. Die Geltung des hier produzierten Wissens beschränkt sich folglich nicht auf einzelne Organisationen oder gesellschaftliche Bereiche, sondern hat eine gesamtgesellschaftliche Relevanz. ${ }^{69}$ Die Wissensproduktion hat eine unumgänglich politische und damit rechtfertigungsbasierte Dimension, da das hier produzierte Wissen die Gesellschaft als Ganzes betrifft. Dasselbe gilt auch für Erhebungen der akademischen Survey-Welt. Die hier angestrebte Wissensform bezieht sich nicht nur auf einzelne Situationen, sondern sucht nach grundsätzlichen Aussagen über soziale Mechanismen. Die generischen Welten orientieren sich nicht an den einzelnen Datennutzenden, sondern an deren Kollektiv (Storper 1997). Diese Orientierung an der Kollektivität wird in der Welt der Information besonders deutlich, in welcher die Kategorien vor dem eigentlichen Interesse der Datennutzenden an den Daten bereits festgelegt und auch die Erhebung vor dem spezifischen Interesse von Datennutzenden durchgeführt wird. Dadurch stehen für den Datennutzenden bei einem Informationsbedürfnis bereits fertige Daten zur Verfügung, welche allerdings von seinem spezifischen Interesse abstrahieren.

Da sowohl die akademische Welt wie auch die Welt der Information dem generischen Pol zuzuordnen sind, kann nach dem Verhältnis der beiden Welten zueinander gefragt werden. Tatsächlich sind diese nicht unabhängig voneinander zu denken. So sehen auch Storper und Salais in der Welt der intellektuellen Ressourcen eine Ressource für andere Welten (1997, S. 37). Damit ist nicht gemeint, dass es hier viele Beispiele von Survey-Projekten gibt, welche als Mischformen beider Welten betrachtet werden müssen, wie beispielsweise Panel-Studien. Vielmehr soll auf den Umstand verwiesen werden, dass die Ergebnisse der akademischen Welt die Basis für die Erhebungen der Informationswelt darstellen. Die in der Informationswelt verwendeten statistischen Formen haben ihren Ursprung in den Untersuchungen der akademischen Welt, indem sie die Grundlage bilden für ein Verständnis und eine Kategorisierung des Sozialen. Dem muss aber ein Formationsprozess folgen, in welchem die verschiedenen Befunde der akademischen Welt, welche auch gegensätzlich sein können, zu einer statistischen Form

\footnotetext{
${ }^{69}$ Wobei der Gesellschaftsbegriff nicht zwingend an Nationalstaaten gebunden ist.
} 
verdichtet werden. ${ }^{70}$ Die akademische Welt wiederum kann die statistischen Formen der Informationswelt verwenden, um neues Wissen zu generieren, indem sie Abweichungen von den statistischen Formen zu Grunde liegenden Annahmen aufzeigt.

Beim gewidmeten Pol hingegen ist es nicht das Ziel, generelles Wissen zu produzieren, sondern eine Entscheidungsgrundlage für individuelle organisationale Fragestellungen. ${ }^{71}$ Erhebungen in diesen Welten sind folglich auf die spezifische Fragestellung oder das spezifische Problem von Organisationen zugeschnitten und sind dadurch für Außenstehende nur von geringer Bedeutung. Durch diese individualisierte und spezifische Forschung wären auch veröffentlichte Daten von geringem allgemeinem Interesse.

Bei den Welten, welche dem spezialisierten Pol zugerechnet werden können, geht es um die Erarbeitung neuer Theorien, methodischer Zugriffe und Konzepte. Existierendes Wissen wird hier infrage gestellt und es wird versucht, dieses durch neues Wissen zu ersetzen (Storper und Salais 1997, S. 30 f.). Der spezialisierte Pol kann deswegen als Entdeckungspol bezeichnet werden, indem hier in kritischer Manier konstant neue Wissenskonzepte und Theorien hervorgebracht werden. ${ }^{72}$

Die Welten des standardisierten Pols sind durch eine konservative Perspektive auf die gesellschaftliche Dynamik gekennzeichnet, welche mit der Standardisierung der Erhebungsinstrumente (nicht der Resultate) einhergeht. So wird beispielsweise in der Dienstleistungswelt angenommen, dass die hier (standardisiert) angebotenen Produkte die operativen Fragen von Unternehmen zufriedenstellend

\footnotetext{
${ }^{70}$ Und genau dies stellt den politischen Prozess der Formgebung dar, indem im wahrsten Sinne des Wortes „Fakten“ geschaffen werden müssen. Vgl. hierzu einen Leitfaden zu Indikatorprojekten (LUSTAT Statistik Luzern 2012). Desrosières beschreibt dieses Schaffen von Fakten als eine Verwandlung von konventionellen hin zu realistischen Daten (2009a). Dieser Effekt ist in der Informationswelt am größten, da hier eine allgemeingültige Information produziert werden soll.

${ }^{71}$ Dieser Aspekt von Survey-Welten ist mit dem Konzept der Produktionswelten nicht vergleichbar, da aus der Perspektive der Produktionsweltentheorie auch Privatpersonen Kunden sein können, während dies im Hinblick auf die Survey-Produktion kaum der Fall ist. Dies wird besonders deutlich in der Darstellung der interpersonellen Welt (Storper und Salais 1997, S. 35).

${ }^{72}$ Vgl. zu einer Unterscheidung zwischen Entdeckungs- und Realitätspol auch Desrosières (1979). Desrosières zeigt hier auf, wie die Ökonomie auf etablierten Konzepten aufbaut und diese erfolgreich in die Öffentlichkeit überführt, währen die Soziologie eine häretische Wissenschaft darstellt, welche in der Folge als Formgeber in der Öffentlichkeit konstant untervertreten ist.
} 
beantworten können und dass die verschiedenen Produkte der Survey-Firmen untereinander vergleichbar sind (Storper und Salais 1997, S. 31). Eine Annahme, welche die Beratungswelt nicht trifft, da sie am Gegenstand das richtige methodische Vorgehen entwickelt. Auch die Welt der Information geht davon aus, dass die verwendeten statistischen Formen die Realität wie angenommen abbilden. Im Vergleich zum Forschungspol kann der standardisierte Pol deswegen als Realitätspol bezeichnet werden. Realität wird hier nicht stets aufs Neue untersucht und konzipiert, sondern eingesammelt und kartografiert.

Wie in den Darstellungen der verschiedenen Survey-Welten aufgezeigt wurde, können die unterschiedlichen Produktionsformen verschiedener Wissensformate als unterschiedliche Konventionen der Survey-Forschung beschrieben werden. Hierzu lassen sich (wie beschrieben) auch typische Produkte für jede Produktionswelt finden, bei welcher also die Konvention der Survey-Forschung am deutlichsten zutage tritt. ${ }^{73}$ Durch die Darstellung der typischen Produkte stellt sich natürlich die Frage, welche Erklärungskraft das Konzept der Survey-Welten für Survey-Projekte hat, welche zwischen den Welten liegen und somit keiner Survey-Welt exklusiv zugeschrieben werden können. ${ }^{74}$ Diese sind im Konzept der Survey-Welten keineswegs nicht vorgesehen oder werden als problematisch erachtet. Sie sind vielmehr der Idealfall, da auch eigentlich typische Produkte einer Survey-Welt oft Mischungen aus verschiedenen Welten darstellen. So werden beispielsweise auch in Universitätsstudien „standardmäßig“ demografische Informationen erfragt. Dies sind klassische statistische Formen, also eigentlich der Survey-Welt der Information zuzuschreiben. Und so kommen auch Survey-Firmen, welche eher der Beratungswelt zugeteilt werden können, kaum ohne eine Angebotsliste aus, welche eigentlich ein Vermarktungsinstrument der Dienstleistungswelt darstellt. Denn auch in dieser Welt will sich der Kunde einen Überblick über die Tätigkeiten der Survey-Firma informieren. Die Mischung möglicher Survey-Welten in Survey-Projekten ist die Regel und nicht die Ausnahme. Im Folgenden soll anhand ausgewählter Beispiele die Koordinationsproblematik in solchen Projekten dargestellt werden.

\footnotetext{
${ }^{73}$ Hierzu findet sich kein Äquivalent in der Produktionsweltentheorie, da beispielsweise die Lederindustrie in Italien infolge einer unterschiedlichen Produktionsorganisation auch in der industriellen Welt angesiedelt werden könnte (Storper und Salais 1997, S. 149 ff.).

${ }^{74}$ Auch aus der Perspektive der Produktionsweltentheorie existieren Kompromisse zwischen Welten, welche sich folglich in Kompromissprodukten niederschlagen (Salais und Storper 1992, S. 186 f.).
} 
Eine von der Wirtschaft und auch von Universitäten und Hochschulen favorisierte Methode des stufenlosen Übergangs von Studierenden ins Erwerbsleben sind Praktika oder eine Zusammenarbeit mit wirtschaftlichen Akteuren bei Abschlussarbeiten. Im Falle der quantitativen Sozialwissenschaften können dies Surveys sein, welche hier als „Werkstudierenden-Surveys“ bezeichnet werden. ${ }^{75}$ Gemeinsam ist dieser Form von Surveys, dass sie ein Problem für einen wirtschaftlichen Akteur lösen sollen, gleichzeitig aber den Kriterien der Wissenschaft genügen. Hier kann beispielhaft aufgezeigt werden, was viele mit einem solchen Projekt beschäftige Studierende aus Erfahrung wissen, nämlich dass dies kein einfaches Unterfangen darstellt. Denn wie anhand der Beratungs- und der akademischen Welt aufgezeigt werden kann, widersprechen sich die Erkenntnisabsichten der beiden Welten. Folgt der Studierende der Konvention der akademischen Welt, wird er mit seinem Survey eine Aussage anstreben, welche sich auf den akademischen Diskurs bezieht und dadurch allgemeine Aussagen hervorbringt. Dann stellt sich für den wirtschaftlichen Akteur jedoch das Problem, dass er allgemeine Aussagen als Resultat der Arbeit geliefert bekommt, die er dann selber auf seine Fragestellung und spezifische Situation beziehen muss, wodurch eventuell weiteres Datenmaterial notwendig wäre. Orientieren sich Studierende jedoch an der Beratungswelt, so werden sie mit der akademischen Kritik konfrontiert, dass die von ihnen getätigten Aussagen zu partikular seien und nicht über den untersuchten Fall hinausgehen würden. Zentral für das produktionsweltliche Argument ist dabei, dass sich die problematische Vermittlung beider Welten nicht als alleinigen Zielkonflikt darstellt. Der methodische Unterschied zwischen beiden Survey-Welten mündet also nicht in der alleinigen Frage danach, auf wen die Arbeit zu den Resultaten des Werkstudierenden-Surveys zugeschrieben werden soll. Vielmehr stellen sich bereits früher methodische Fragestellungen.

Einen Kompromiss zwischen der Beratungs- und der Dienstleistungswelt gehen „Drittanbieter-Studien“ ein. Drittanbieter-Studien sind Erhebungen, welche in der Art der Dienstleistungswelt als Auftrag an die Survey-Firma formuliert werden, ihren Ursprung jedoch in der Beratung von Klienten durch Drittanbieter, d. h. nicht Survey-Firmen, haben. Wie sich während den Feldforschungen zeigte, kommt diese Art der Auftragserteilung verhältnismäßig häufig zustande, indem sich Organisationen durch andere Organisationen beraten lassen, welche in der Folge eine survey-basierte Erhebung als Teil der Beratung bei einer Survey-Firma in Auftrag geben. Durch diese Dritt-Organisationen muss in der Folge eine Übersetzung einer individuellen Problemstellung hin zu einem standardisierten Angebot der Survey-Firma geleistet werden.

\footnotetext{
${ }^{75}$ Die folgenden Ausführungen beziehen sich auf Daten der teilnehmenden Beobachtung.
} 
Zwischen Dienstleistungs- und Informationswelt gelegen sind „BefragungsProdukte“. Diese sind wie in der Dienstleistungswelt standardisiert, darüber hinaus jedoch beispielsweise zwischen verschiedenen Tochterunternehmen in verschiedenen Ländern gleichzeitig auch uniformiert. Die Daten werden hierbei nicht wie in der Informationswelt bereits vor der eigentlichen Anfrage von Kunden erhoben, dennoch ermöglicht die Uniformierung eine hohe Vergleichbarkeit mit anderen Erhebungen, welche gemäß der Methodik desselben Befragungs-Produkts erhoben worden sind. Die Koordinationsproblematik in solchen Erhebungen liegt darin, auf der einen Seite individuelle Wünsche und Erwartungshaltungen abdecken zu können und auf der anderen Seite immer noch eine hohe Vergleichbarkeit der Resultate garantieren zu können.

„Panel-Studien“ werden folgend als Kompromissprodukte zwischen der akademischen Welt und der Informationswelt analysiert. Dies soll nicht darüber hinweg täuschen, dass der tatsächliche informationsweltliche Einfluss bei verschiedenen Panel-Studien unterschiedlich sein kann und Panel-Studien grundsätzlich eher an der akademischen Survey-Welt orientiert sind. Nichtsdestotrotz besteht in sozialwissenschaftlichen Panels die Problematik, dass sich diese aufgrund von deren Kapital- und Ressourcenintensivität kaum an einen Forschungsansatz alleine richten, sondern grundsätzlich an ein Forschungskollektiv. Damit werden Panel-Studien quasi durch die Hintertür informationsweltlich geentert, da die notwendige Kollektivität - wenn auch auf einem eher tiefen Level im Vergleich beispielsweise zu staatlichen Statistiken - zu einer Konzentration auf etablierte Erhebungskategorien im entsprechenden Forschungsgebiet führt. Ein informationsweltlicher Aspekt von Panel-Studien und von akademisch orientierten Erhebungen allgemein stellt auch der Rückgriff auf allgemeine demografische Kategorien dar. Die hier erhobenen Kategorien müssen nicht zwangsweise Kategorien der zugrunde liegenden Forschungsperspektiven sein, ermöglichen jedoch eine schnelle informationsweltliche Übersetzungsleistung, indem die Ergebnisse auf Formen mit einer hohen Reichweite bezogen werden können. Die Koordinationsproblematik bei Panel-Studien, welche einen Kompromiss zwischen der akademischen Welt und der Informationswelt etablieren, besteht darin, einerseits kollektiv akzeptierte Erhebungskategorien zu konzipieren, welche aber zugleich noch eine Erhebung auf Basis von neuen Theorien und Konzepten, bzw. die Überprüfung von ungeklärten Hypothesen ermöglichen. In Abb. 5.10 sind verschiedene Beispiele von typischen „Produkten“ der verschiedenen Survey-Welten dargestellt, wie auch die beschriebenen Kompromissprodukte zwischen verschiedenen Survey-Welten.

Im Folgenden wird ein tabellarischer Überblick und Vergleich zwischen den verschiedenen Survey-Welten dargestellt. Durch die intensive Aufarbeitung der verschiedenen Survey-Welten in den einzelnen vorhergehenden Unterkapiteln 


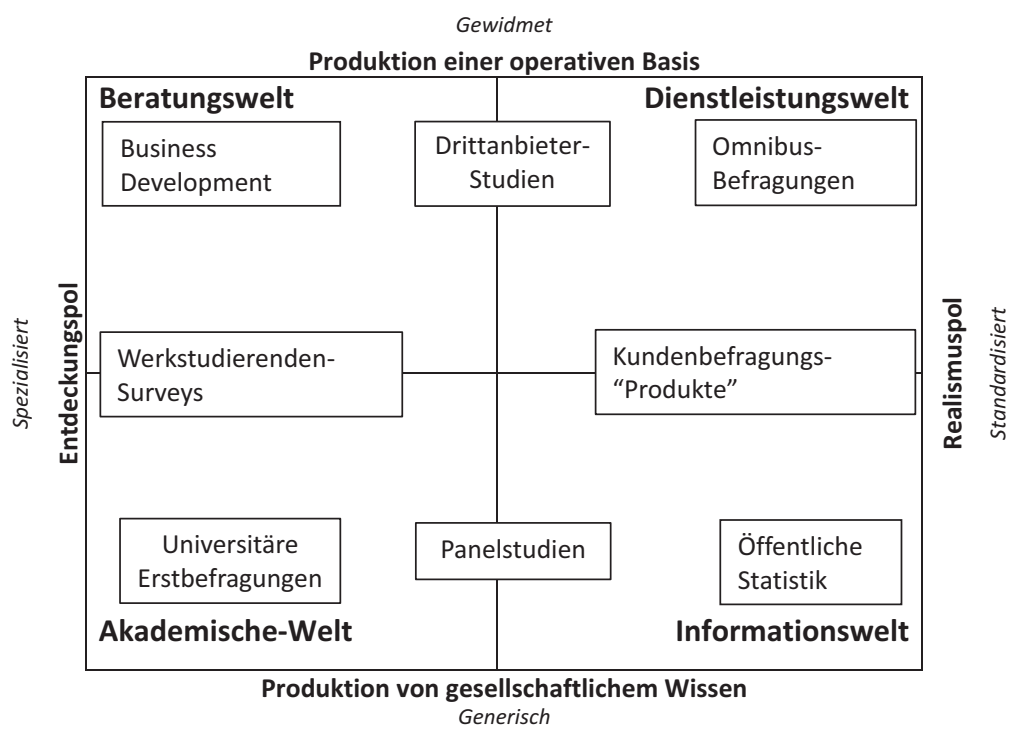

Abb. 5.10 Survey-Welten im Überblick

erübrigt sich eine intensive Diskussion der verschiedenen Vergleichsdimensionen. Im Hinblick auf die einzelnen Dimensionen sei deshalb auf die einzelnen Kapitel zu Survey-Welten verwiesen. Die Thematik der Zeitlichkeit und der zeitlichen wie räumlichen Reichweite wurde jedoch in den entsprechenden Unterkapiteln nur am Rande thematisiert, weswegen hier ausführlicher darauf eingegangen wird.

Zeitlich ist die Beratungswelt an organisationalen Problemen orientiert. Die Forschung findet hier folglich punktuell und in Wellen statt, da survey-basierte Befragungen dann durchgeführt werden, wenn eine Neuorientierung einer Unternehmensstrategie, bzw. der Strategie einzelner Abteilungen in einem Unternehmen ansteht. Dadurch, dass in dieser Welt nicht einzelne, durch das Unternehmen vorformulierte Kategorien befragt werden, sondern durch beratungsorientierte Survey-Firmen eine reflexive Perspektive auf ein spezifisches organisationales Problem eingeführt wird, besteht hier eine mittlere zeitliche Reichweite. Denn hier werden - im Unterschied zur Dienstleistungswelt - keine tagesaktuellen Fragestellungen beantwortet, welche schnell wieder ihren Wissensgehalt verlieren können, sondern es findet eine wissens-organisatorische Umwälzung innerhalb einer Organisation statt, was eine erhöhte Reichweite im Vergleich zur Dienstleistungswelt zur Folge hat. Durch die Spezifität 
der Fragestellung besteht jedoch eine nur geringe räumliche Reichweite der hier produzierten Daten, d. h. ein geringes Maß eines kollektiven Informationswertes (Thévenot 2011d). Im Hinblick auf die räumliche Reichweite stellt diese Welt folglich eine Antithese zum Konzept des Äquivalenzraums von Desrosières dar (Desrosières 2005).

Die Dienstleistungswelt hingegen ist zeitlich an wiederkehrenden organisationalen Fragestellungen orientiert. Wie bereits dargestellt ist die zeitliche Reichweite dadurch gering, da das Wissen spezifisch für die Lösung aktueller Fragestellungen dient. Die räumliche Reichweite hingegen ist durch die Standardisierung des Marktangebotes im Vergleich zur Beratungswelt erhöht, da eine gewisse Vergleichbarkeit zwischen den verschiedenen Erhebungen gegeben ist.

Die Zeitorientierung der Forschung ist an zeitlichen Formen mit einer hohen Reichweite wie beispielsweise Jahren, Quartalen oder Monaten ausgerichtet. Diese Orientierung trägt zu einer weiteren Erhöhung der hier produzierten Informationen bei. Die zeitliche wie auch räumliche Reichweite ist hier hoch und im Vergleich zu den anderen Survey-Welten am ausgeprägtesten. Damit entspricht diese Welt dem Konzept des Äquivalenzraums von Desrosières (Desrosières 2005). Die zeitliche Reichweite wird durch die konservative Beibehaltung von Erhebungskategorien über die Zeit erreicht, wodurch Änderungen dieser Kategorien in dieser Welt immer problematisch sind und zurückhaltend umgesetzt werden. Die räumliche Reichweite wird auf zwei Wegen erreicht. Einerseits findet eine generelle Orientierung an in der Öffentlichkeit etablierten Formen statt. Zusätzlich findet aber auch eine gezielte Investition in Formen statt, wie beispielsweise durch das Marketing von Informationsintermediären (Bessy und Chauvin 2013) oder durch die staatliche Durchsetzung von Kategorisierungen (Desrosières 2000).

Die akademische Survey-Welt ist grundsätzlich durch das Bestreben gekennzeichnet, eine zeitlose Forschung zu betreiben, indem allgemeine Zusammenhänge aufgezeigt werden. Durch die starke Orientierung am wissenschaftlichen Diskurs können diese Forschungsbestrebungen jedoch schnell wieder egalisiert werden. Entsprechend ist die Zeitlichkeit der Resultate - analog zur Informationswelt, aber im Unterschied zur Beratungs- und Dienstleistungswelt - nicht nur durch eigene Survey-Erhebungen limitiert, sondern auch durch Erhebungen durch andere Forschende. Durch die hohe nationale und internationale Vernetzung von Forschenden, aber auch Theorieströmungen, haben die hier produzierten Daten eine hohe räumliche Reichweite. Die zeitliche Reichweite ist hingegen aufgrund der bereits beschriebenen Wissenschaftsdynamik beschränkt.

In Tab. 5.2 sind die Unterschiede zwischen den Survey-Welten im Hinblick auf verschiedene Konventionen tabellarisch zusammengefasst. 


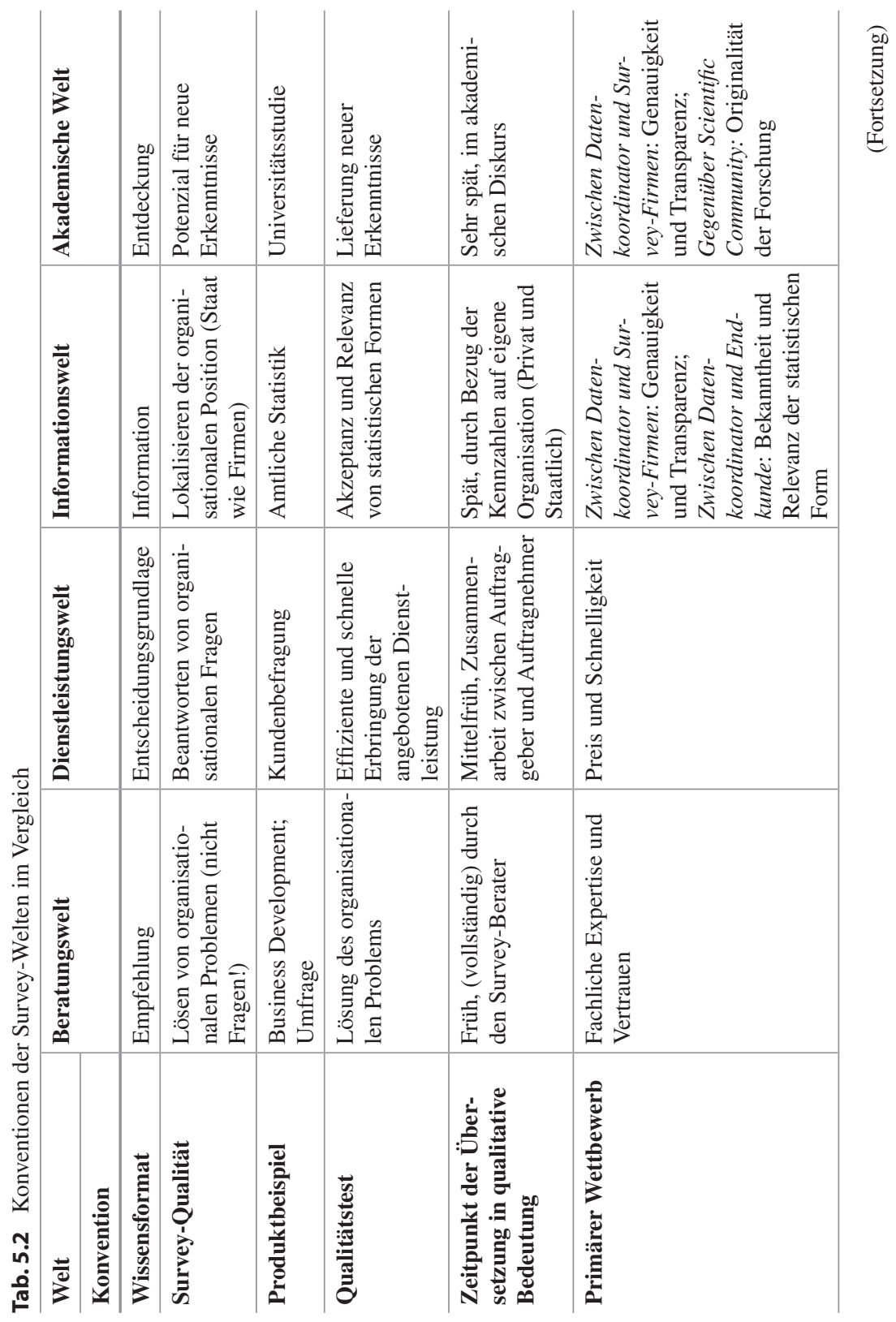




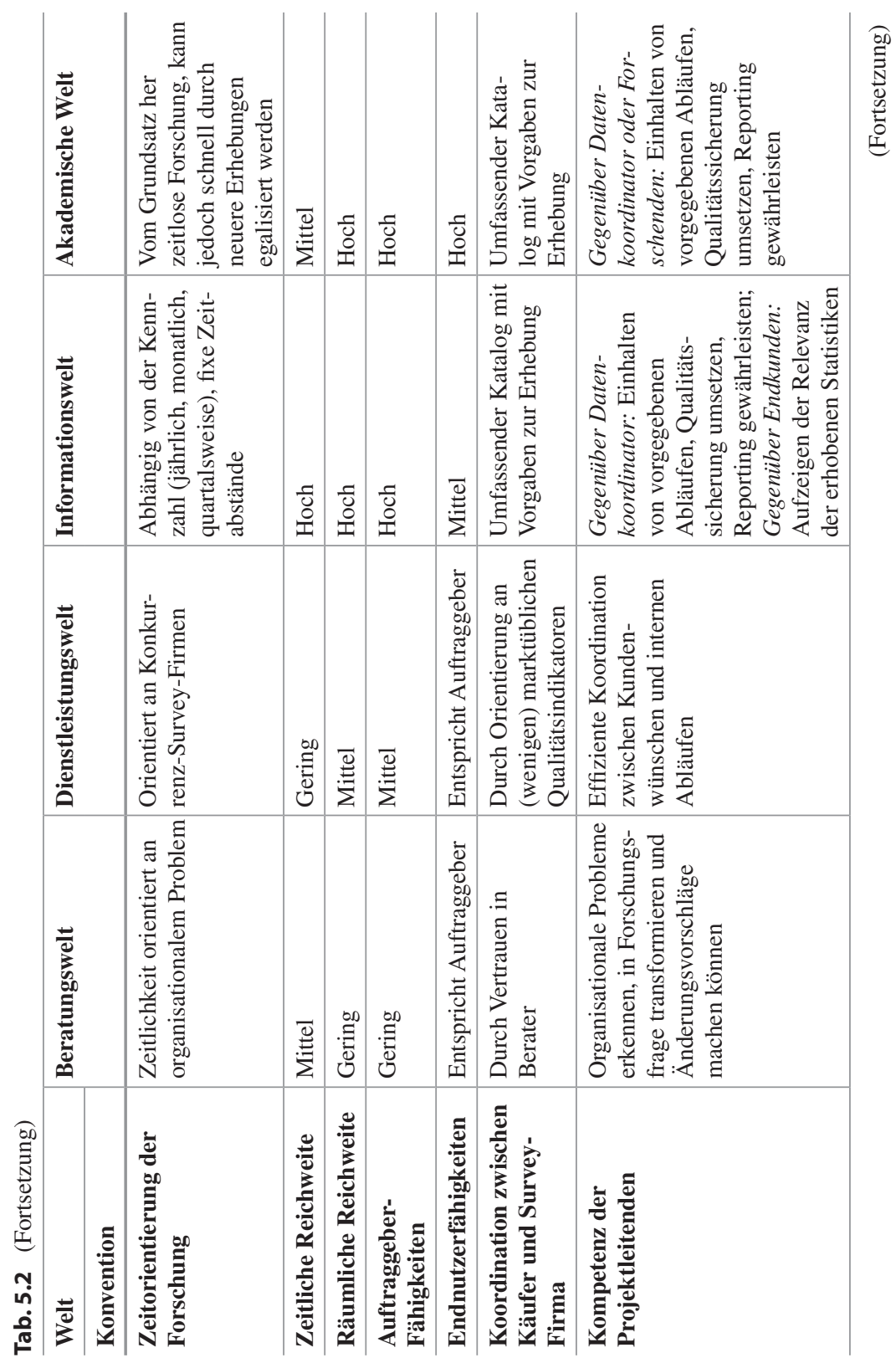




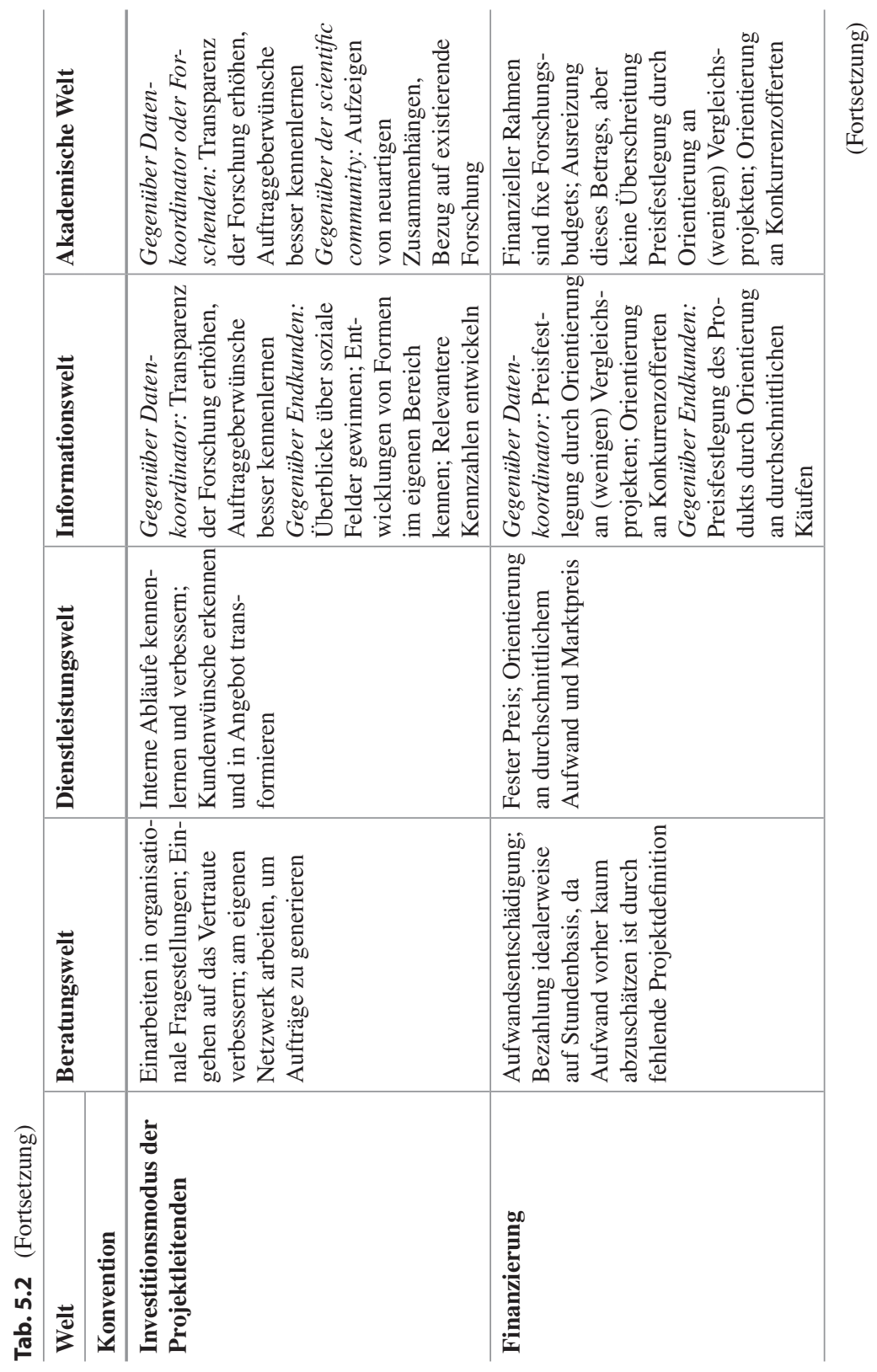




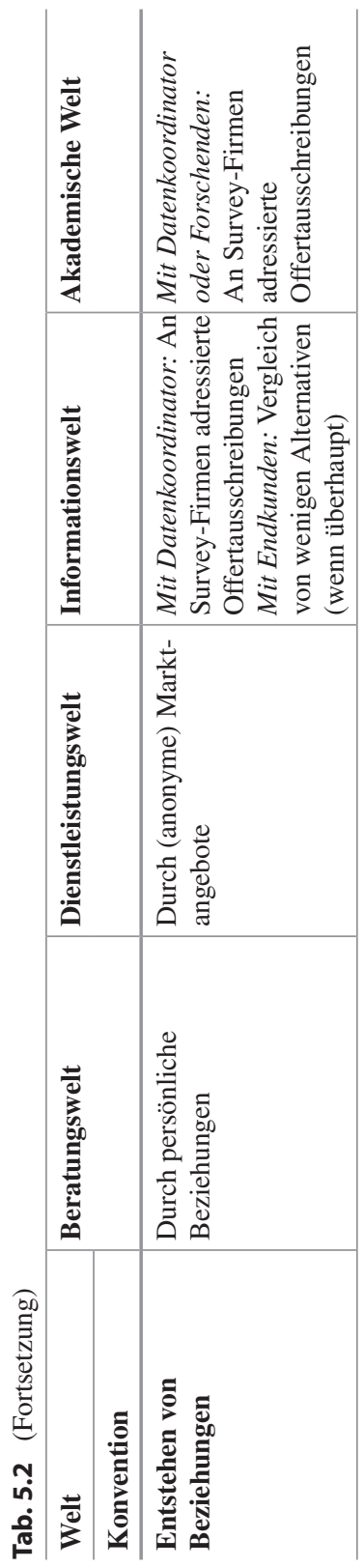


Open Access Dieses Kapitel wird unter der Creative Commons Namensnennung 4.0 International Lizenz (http://creativecommons.org/licenses/by/4.0/deed.de) veröffentlicht, welche die Nutzung, Vervielfältigung, Bearbeitung, Verbreitung und Wiedergabe in jeglichem Medium und Format erlaubt, sofern Sie den/die ursprünglichen Autor(en) und die Quelle ordnungsgemäß nennen, einen Link zur Creative Commons Lizenz beifügen und angeben, ob Änderungen vorgenommen wurden.

Die in diesem Kapitel enthaltenen Bilder und sonstiges Drittmaterial unterliegen ebenfalls der genannten Creative Commons Lizenz, sofern sich aus der Abbildungslegende nichts anderes ergibt. Sofern das betreffende Material nicht unter der genannten Creative Commons Lizenz steht und die betreffende Handlung nicht nach gesetzlichen Vorschriften erlaubt ist, ist für die oben aufgeführten Weiterverwendungen des Materials die Einwilligung des jeweiligen Rechteinhabers einzuholen.

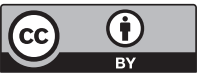

